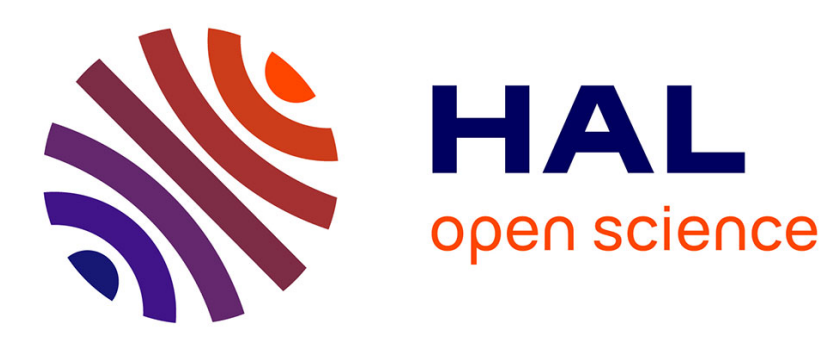

\title{
Validity of quasilinear theory: refutations and new numerical confirmation
}

\author{
Nicolas Besse, Yves Elskens, Dominique Escande, Pierre Bertrand
}

\section{To cite this version:}

Nicolas Besse, Yves Elskens, Dominique Escande, Pierre Bertrand. Validity of quasilinear theory: refutations and new numerical confirmation. 2010. hal-00527149

\section{HAL Id: hal-00527149 \\ https://hal.science/hal-00527149}

Submitted on 18 Oct 2010

HAL is a multi-disciplinary open access archive for the deposit and dissemination of scientific research documents, whether they are published or not. The documents may come from teaching and research institutions in France or abroad, or from public or private research centers.
L'archive ouverte pluridisciplinaire HAL, est destinée au dépôt et à la diffusion de documents scientifiques de niveau recherche, publiés ou non, émanant des établissements d'enseignement et de recherche français ou étrangers, des laboratoires publics ou privés. 


\title{
Validity of quasilinear theory: refutations and new numerical confirmation
}

\author{
Nicolas Besse ${ }^{1}$, Yves Elskens ${ }^{2}$, D F Escande ${ }^{2}$ and Pierre \\ Bertrand $^{1}$ \\ ${ }^{1}$ UMR 7198 CNRS-Nancy Universités, Université Henri Poincaré, \\ Bd. des Aiguillettes, B.P. 70239, FR-54506 Vandœuvre-lès-Nancy cedex \\ E-mail: nicolas.besse@iecn.u-nancy.fr \\ ${ }^{2}$ UMR 6633 CNRS-Université de Provence, Faculté de St Jérôme, case 321, \\ Av. Normandie Niemen, FR-13397 Marseille cedex 20 \\ E-mail: yves.elskens@univ-provence.fr, dominique.escande@univ-provence.fr
}

\begin{abstract}
.
Over almost three decades an unsettled controversy developed about the validity of quasilinear (QL) theory for describing the weak warm beam-plasma instability in the chaotic saturation regime. This work deals both analytically and numerically with this issue. It is shown analytically that there is no mode coupling in the saturation regime of the instability where a plateau is present in the tail of the particle distribution function. This invalidates several analytical works trying to prove or to contradict the validity of quasilinear estimates in the strongly nonlinear regime of the weak warm beamplasma instability. Self-consistent numerical simulations of the weak warm beamplasma instability within the Vlasov-wave description show that QL theory remains valid in the strong chaotic diffusion regime. However there is a non-QL regime before saturation, which confirms another analytical work and contradicts a later one, but confirms a previous numerical simulation. [18 October 2010]
\end{abstract}

PACS numbers: 05.20.Dd, 52.25.Dg, 52.25.Gj, 52.35.Fp, 52.35.Qz, 52.35.Ra, 05.45.Jn Keywords: Quasilinear theory, Vlasov equation, Fokker-Planck equation, diffusion, kinetic turbulence, bump-on-tail instability, chaotic regime, wave-particle interaction.

Submitted to: Plasma Phys. Control. Fusion 


\section{Introduction}

Wave-particle interaction is an important phenomenon in plasma physics. It stands at the root of wave heating, of numerous instabilities, and of some regimes of anomalous transport in magnetically confined plasmas. Wave-particle interaction, described by Vlasov-Maxwell equations or "simply" by one-dimensional Vlasov-Poisson equations, is already so complex that Landau damping was fully recognized only after its experimental discovery in 1964 [44], even though it was discovered on an analytical basis in 1946 [37]. As to nonlinear description, the situation is worse and has led to a controversy recalled below about the validity of the quasilinear (QL) equations for describing the saturation of the weak warm beam-plasma instability. Furthermore QL estimates are quite frequently used for modelling in different branches of plasma physics, such as laserplasma interaction or magnetized plasma turbulence. Since the QL approximation is ubiquitous, in particular in kinetic or gyrokinetic descriptions, it is important to assess its validity at least for the simplest problem of kinetic turbulence, i.e. the saturation of the weak warm beam-plasma instability. We now sketch this problem (see [26] for an intuitive introduction and chapter 7 of [21] for a more exhaustive one).

\subsection{Formulation of the problem}

We consider a two-dimensional distribution function of particles in $(x, v)$ space which is initially given for a one-dimensional spatially uniform beam-plasma system. This beam corresponds to a bump on the tail of the electron velocity distribution function. Langmuir waves are destabilized by the inversion of the electron population corresponding to the positive slope interval of the distribution. They first grow linearly, but when the electron dynamics becomes chaotic enough in their range of phase velocities, the bump is eroded and eventually a plateau in the distribution function builds up. Simultaneously, there is a transfer of momentum from the particles to the waves, generating a turbulent spectrum of Langmuir waves. This scenario was first predicted on a theoretical basis $[55,16]$ by considering the wave-particle interaction as perturbative and neglecting all mode couplings in the Vlasov equation, except for their effect on the space averaged distribution function $f$. This led to the set of QL equations coupling $f(t, v)$ and the waves field power spectrum $\psi(t, v)$, which is related (see (20)) to the ( $k$-density of the) Poynting vector of the waves electric field:

$$
\begin{aligned}
& \partial_{t} f=\partial_{v}\left(D_{\mathrm{QL}}(t, v) \partial_{v} f\right), \\
& \partial_{t} \psi=2 \gamma_{\mathrm{L}}(t, v) \psi,
\end{aligned}
$$

where

$$
\gamma_{\mathrm{L}}(t, v)=\frac{\pi}{2} \frac{\eta}{1+\eta} \frac{\omega_{\mathrm{p}}^{3}}{k^{2}} \partial_{v} f(t, v)
$$

is the Landau growth rate computed with the instantaneous velocity distribution, while

$$
D_{\mathrm{QL}}(t, v)=\pi \frac{\eta}{1+\eta} \frac{\psi(t, v)}{k^{2}}
$$


is the QL diffusion coefficient. In these formulæ, $\omega_{\mathrm{p}}$ is the plasma frequency, and $\eta=n_{\mathrm{b}} / n_{\mathrm{p}}$ denotes the ratio of the beam density $n_{\mathrm{b}}$ (the tail particle distribution function) to plasma density $n_{\mathrm{p}}$ (the bulk particle distribution function). Note that phase velocity $v$ and wavenumber $k$ are linked by $v=\omega / k$ and the Bohm-Gross dispersion relation $\mathcal{D}(k, \omega)=0$ viz.

$$
\omega^{2} / \omega_{\mathrm{p}}^{2}=1+3 k^{2} \lambda_{\mathrm{D}}^{2}
$$

with $k \lambda_{\mathrm{D}} \ll 1$ where $\lambda_{\mathrm{D}}$ is the Debye length. We focus on one-dimensional dynamics with periodic boundary conditions, so that the wave spectrum is discrete.

\subsection{Some controversial issues}

Later on, the QL scenario was confirmed experimentally [50], but with poor accuracy for $\gamma_{\mathrm{L}}$ and $D_{\mathrm{QL}}$ in the saturation regime. However the quasi-ballistic assumption underlying QL calculations is incorrect in the saturation regime [1], as is recalled now. If at time $t_{0}$ velocity diffuses with a diffusion coefficient $D_{\mathrm{QL}}$ assumed to be independent of time for the moment, a particle velocity and position are stochastic processes, for which we denote by $\langle\cdot\rangle$ the expectations with respect to the law of the random noise. In particular, $\left\langle\Delta v^{2}\right\rangle \simeq 2 D_{\mathrm{QL}}\left(t-t_{0}\right)$ with $\Delta v(t)=v(t)-v\left(t_{0}\right)$. Consequently particle positions spread as $\left\langle k^{2} \Delta x^{2}\right\rangle=\frac{2}{3} k^{2} D_{\mathrm{QL}}\left(t-t_{0}\right)^{3}$ for $\Delta x(t)=\int_{t_{0}}^{t} \Delta v(\tau) \mathrm{d} \tau$. Therefore, positions spread over a typical wavelength of the turbulent wave spectrum when $\left\langle k^{2} \Delta x^{2}\right\rangle \simeq 4 \pi^{2}$, which occurs for a time $\sim t_{0}+\tau_{\text {spread }}$ with $\tau_{\text {spread }}=\left(6 \pi^{2} k^{-2} D_{\mathrm{QL}}{ }^{-1}\right)^{1 / 3} \simeq 4 \tau_{\mathrm{D}}$, where

$$
\tau_{\mathrm{D}}=\left(k^{2} D_{\mathrm{QL}}\right)^{-1 / 3}
$$

is called the Dupree time [17], its reciprocal being the resonance-broadening frequency.

In order to describe completely the classical regime, noted regime $\mathrm{L}$ (inear) in this work, where the original QL approximation is valid, we need to introduce other time scales. Let $\Delta v_{\text {spec }}$ be the phase velocity width of the wave spectrum and $\Delta v_{\varphi}$ be the typical phase velocity mismatch between neighbouring waves. For a typical wave number $k$, we then define the wave autocorrelation time $\tau_{\mathrm{ac}}=\left(k \Delta v_{\mathrm{spec}}\right)^{-1}$, viz. the time needed for a resonant particle to resolve the finite frequency width of the wave spectrum, and the discretization time $\tau_{\text {discr }}=\left(k \Delta v_{\varphi}\right)^{-1}$, viz. the time it takes a resonant particle to resolve the separate Doppler frequencies of the modes. Particles released at time $t_{0}$ have their velocity spread $\Delta v$ that grows linearly with time for $t$ below $t_{0}+\tau_{\text {ac }}$, and $\left\langle\Delta v^{2}\right\rangle$ grows quadratically, since all waves act with an almost constant force on the particle orbit during this time interval. Therefore there is no diffusion. For time beyond $\tau_{\text {discr }}$, the dynamics feels the discreteness of the wave spectrum, and the perturbative motion, if physically relevant because $\tau_{\text {spread }} \gg \tau_{\text {discr }}$, is no longer diffusive but may be quasi-periodic. We now define the dimensionless parameters

$$
\mu=\left(\gamma_{\mathrm{L}} \tau_{\mathrm{D}}\right)^{-1}, \quad K_{\mathrm{D}}=\tau_{\mathrm{ac}} / \tau_{\mathrm{D}}, \quad \mathcal{B}=\tau_{\mathrm{D}} / \tau_{\text {discr }},
$$

where $K_{\mathrm{D}}$ is called the Kubo number. For a small enough initial amplitude of the waves and a correspondingly small enough $\Delta v_{\varphi}$, the initial QL regime (regime L) is 
characterised by $\mu \ll 1, K_{\mathrm{D}} \ll 1$ and $\mathcal{B} \ll 1$. The parameter $\mathcal{B}$ is linked to the Chirikov resonance overlap parameter $s_{\text {ov }}=2 \Delta v_{\text {trap }} / \Delta v_{\varphi}$ by the relation $\mathcal{B}=8 \pi^{-1 / 3} s_{\text {ov }}^{-4 / 3}$ where $\Delta v_{\text {trap }}$ is the typical trapping width of a wave. Therefore $\mathcal{B} \ll 1$ means $s_{\text {ov }} \gg 1$. The condition $\mu \ll 1$ cannot be satisfied during the whole saturation regime of the beamplasma instability since the plateau formation means ultimately a vanishing slope of $f(t, v)$ and a vanishing $\gamma_{\mathrm{L}}(t, v)$. Therefore there is a crossover to the strongly nonlinear regime $\mu \gg 1$. Indeed strong mode coupling is seen both numerically and experimentally (see $[15,12,38,41]$ and references therein). However, though the QL assumption ceases to hold, the central question about the validity of QL equations remains unsolved in the strong nonlinear regime, denoted SNL and characterized by $\mu \gg 1, K_{\mathrm{D}} \ll 1$ and $\mathcal{B} \ll 1$.

From a theoretical point of view, the validity of quasilinear theory was questioned by Adam et al. [1] when accounting for nonlinear wave coupling. The importance of this coupling was denied by Galeev et al. [35]. In 1983, Laval and Pesme predicted an increase of the growth rate and diffusion coefficient with respect to their QL values when $\mu$ increases [38], and claimed the inconsistency of quasilinear theory due to mode coupling [39]. They proposed a model predicting a renormalization by a factor 2.2 of the quasilinear growth rate and diffusion coefficient on the basis of a "turbulent trapping" Ansatz (derived from a clump-theory-like approach [17]) in the nonlinear regime $\mu \gg 1$ [40], an improved version of their previous claim [1]. Aimed at checking this prediction, a first experiment reached a weakly nonlinear regime where mode coupling was strong, but no renormalization was found [54]. The difficulty of dealing analytically with strongly nonlinear regimes of the Vlasov-Poisson system motivated a new mechanical approach, which was further encouraged by progress in the understanding of lowdimensional Hamiltonian chaos [21]. The experiment of Tsunoda et al. [54] further fuelled the ongoing controversy which produced about twenty analytical, numerical and experimental works over two decades. In particular, Liang and Diamond [42, 43] stated the turbulent trapping model to be inconsistent because momentum conservation is violated. They proposed a derivation of the quasilinear equations in the $\mu \gg 1$ regime by using the theory of two-point correlation functions initially proposed by BoutrosGhali and Dupree [11]. Shapiro and Sagdeev [51], investigating the four-wave coupling, asserted that quasilinear theory works if the particle distribution function and the wave spectrum are averaged over a width in velocity defined by $\left\langle\Delta v^{2}\right\rangle \simeq 2 D_{\mathrm{QL}} \tau_{\mathrm{D}}=2 \Delta v_{D}^{2}$ where

$$
\Delta v_{\mathrm{D}}=\left(D_{\mathrm{QL}} / k\right)^{1 / 3}=k / \tau_{\mathrm{D}}
$$

is called the Dupree width. References [28, 21] derived quasilinear equations in the $\mu \gg 1$ regime, by extending the technique for the non self-consistent case thanks to the slow effect of one particle on a given wave [28].

As yet no consensus has been reached [41, 21]. Experiments and numerical simulations have not yet described the strongly nonlinear regime in an accurate way but are compatible with weak renormalization effects in the intermediate (nonlinear) 
regime, denoted INL, where $\mu \gtrsim 1, K_{\mathrm{D}} \ll 1$ and $\mathcal{B} \ll 1[12,15,36,41] . \ddagger$

\subsection{Position of this work}

The numerical part of this paper presents complete self-consistent simulations in the Vlasovian framework, starting from regime L to reach regime SNL by passing through regime INL. Before seeing whether self-consistent quasilinear theory is still valid in regime SNL, let us understand why QL equations still hold in the case of non-selfconsistent dynamics with wave spectra like those in regime SNL under some assumptions on the wave spectrum that we will describe further. To understand particle diffusion, it is useful to consider first the case of diffusion in a prescribed set of $M \gg 2$ Langmuir waves with random phases and a smooth amplitude spectrum as defined by the hamiltonian

$$
\mathcal{H}_{\mathrm{nsc}}(t, x, v)=\frac{v^{2}}{2}-\sum_{m=1}^{M} A_{m} \cos \left(k_{m} x-\omega_{m} t+\varphi_{m}\right) .
$$

If the wave spectrum is broad, classical perturbation theory predicts [21, 27, 28, 29, 30] $\left\langle\Delta v^{2}\right\rangle=2 D_{\mathrm{QL}} t$ and $\left\langle k^{2} \Delta x^{2}\right\rangle=\frac{2}{3} k^{2} D_{\mathrm{QL}} t^{3}$ for $\tau_{\mathrm{ac}} \ll t \ll \tau_{\text {pert }}=\min \left(\tau_{\mathrm{D}}, \tau_{\text {discr }}\right)$ where the bracket notation denotes averages over the random phases $\varphi_{m}$. This calculation defines $D_{\mathrm{QL}}$ over a time scale where the spreading of orbits is negligible, and thus chaos is unimportant. Therefore diffusion is due to the randomness of the field, not to the dynamical chaos: this diffusion is stochastic but not chaotic. Note that $\tau_{\text {spread }} \simeq 4 \tau_{\mathrm{D}}$ bounds the time over which the dependence of the orbits over $M$ phases is small.

According to the value of the overlap parameter $s_{\text {ov }}$, several scenarios have been observed numerically for $t>\tau_{\text {pert }}[13,21]$. At small $s_{\text {ov }}$, i.e. for $\mathcal{B} \gg 1$, there is no large-scale chaos and the dynamics first feels the discreteness of the wave spectrum. For $t>\tau_{\text {discr }}$ this dynamics proves to be quasi-periodic and $\left\langle\Delta v^{2}\right\rangle$ saturates; thus the motion is no longer diffusive, which confirms that the initial QL diffusion is not chaotic over large velocity scales. At large $s_{\mathrm{ov}}$, i.e. for $\mathcal{B} \ll 1$, the dynamics feels first the chaotic spreading of the orbits. Then, for $t>\tau_{\text {spread }}$ the dynamics remains diffusive with a diffusion coefficient keeping the QL value. For intermediate values of $s_{\mathrm{ov}}$, where chaos is widespread, the diffusion coefficient $D$ becomes supra-quasilinear for large time: $D \simeq 2.3 D_{\mathrm{QL}}$ for $s_{\mathrm{ov}} \simeq 2.2[13]$. There is a quasilinear non-chaotic diffusion up to time $t=\tau_{\text {pert }}$ and a chaotic non-quasilinear one after $\tau_{\text {pert }}[4,21,28]$. The existence of this intermediate regime shows that QL estimates for the diffusion coefficient at large $s_{\mathrm{ov}}$ do

$\ddagger$ Related work [45, 46] (see also references therein and the physically insightful, well referenced introduction) by Mouhot and Villani, on Landau damping in a nonlinear context, has brought the long awaited mathematical proof of (nonlinear) Landau damping in infinite time with exponential decay, for any interaction not more singular than Coulomb or Newton, including the limit case. As a corollary, they obtain new stability results for homogeneous equilibria of the nonlinear Vlasov-Poisson equation, under analytic perturbations. In particular they show the convergence, in the weak topology in infinite time with exponential rate of convergence, of small enough analytic perturbations towards spatially homogeneous analytic equilibrium profiles which cannot be described in terms of conservation laws and initial datum alone. 
not correspond to a trivial extension of the initial QL regime. Something similar might occur for the regime SNL of the self-consistent case.

As recalled in Appendix A, the origin of chaotic diffusion can be understood as the result of locality in the velocity of the wave-particle interaction. The existence

of a diffusion over time $\tau_{\mathrm{QL}} \gg \tau_{\text {spread }}$ enables the rigorous extension of the validity of quasilinear theory up to the time when orbits hit the Kolmogorov-Arnold-Moser (KAM) boundaries of the chaotic domain, provided that all the phases $\varphi_{m}$ are drawn independently from a uniform distribution on the circle, and the velocity distribution $f$ remains "smooth" (almost constant) over the velocity range $\Delta v_{\text {spec }}$ (no trapping structure) or equivalently the wave spectrum must not be peaked in this velocity (or wavenumber) range [21]: nearby waves may have strongly inhomogeneous amplitudes, but the spectrum should be smooth when averaged over a Dupree width $\Delta v_{\mathrm{D}}$ (8) (or rather a resonance box width, which is about five times larger, see Appendix A). Indeed mode coupling (e.g. modulational instability) can cause the turbulent wave spectrum to become peakier in $k$-space (or velocity space) when it grows; and thus it can generate spatial non-uniformity of the velocity distribution function such as small trapping structures (hole vs hump). In order to show that quasilinear theory remains valid in the chaotic regime SNL to describe the nonlinear self-consistent wave-particle interaction, we will show that, when the plateau is formed in the velocity distribution function, mode coupling becomes negligible, and the dynamics lands in the non-self-consistent stage where the wave spectrum meets the assumptions on which QL estimates rest : the independence of phases and a non-peaked amplitude spectrum. To sum up, the final stage of the nonlinear self-consistent waves-particles evolution must satisfy the following three assumptions: a broad enough plateau regime is well settled (negligible modecoupling for waves whose phase velocity belongs to the plateau), the wave spectrum phases are uniformly distributed and independent, the variations of the wave spectrum amplitude are not peaked in velocity.

\subsection{Structure of this paper}

This paper is organized as follows. Section 2.1 introduces the Vlasov-wave description of the wave-particle interaction. Section 2.2 shows analytically that, if the tail particle distribution displays a plateau, the waves whose phase velocity belongs to the plateau feel a negligible mode-coupling in the limit where the plateau is broad enough. Section 2.3 invalidates previous works attempting to prove or to contradict the validity of quasilinear theory in the saturation regime.

Section 3 describes the numerical scheme to approximate the Vlasov-wave models introduced in section 2.1, and section 4 determines the simulation parameters. Finally sections 5 and 6 present self-consistent simulations within the Vlasov-wave description, where the dynamics go from regime L to regime SNL in which QL estimates remain right and accurate. 


\section{Theoretical framework : description of wave-particle self-consistent dynamics}

The difficulty to describe the nonlinear regime of the Vlasov-Poisson system of equations, and the progress in the chaotic dynamics of Hamiltonian systems with a finite number of degrees of freedom, were an incentive to tackle the description of the saturation regime with the so-called self-consistent Hamiltonian that describes the one-dimensional self-consistent evolution, in a plasma with spatial periodicity $L$, of $M$ Langmuir waves with $N$ particles (the beam) in the tail of the electron distribution function per length $L[21]$.

\subsection{Self-consistent model}

A rigorous classical mechanics calculation [21] allows to reduce the original $N^{*}$-body problem of $N^{*} \gg 1$ electrostatically coupled particles in a one-dimensional periodic system to a field-particle interaction problem incorporating $N$ resonant particles and $M$ harmonic oscillators defining the field, namely the $M$ Langmuir waves due to the collective vibrations of the bulk (non-resonant particles), assuming $N+M \ll N^{*}$.

In the absence of tail particles, the Langmuir waves are the collective motions (eigenmodes) of a plasma (without resonant particles) with a density $n_{\mathrm{p}}$ and with a plasma frequency $\omega_{\mathrm{p}}$. Wave $m$ has pulsation $\omega_{m}$ related to the wavenumber $k_{m}$ through the Bohm-Gross dispersion relation (5). The interaction of these $M$ waves with the $N$ resonant particles is described by the self-consistent Hamiltonian

$\mathcal{H}_{\mathrm{sc}}^{N, M}=\sum_{n=1}^{N} \frac{v_{n}^{2}}{2}+\sum_{m=1}^{M} \omega_{m} \frac{X_{m}^{2}+Y_{m}^{2}}{2}+\tilde{\varepsilon} \sum_{n=1}^{N} \sum_{m=1}^{M} \frac{\beta_{m}}{k_{m}}\left(Y_{m} \sin \left(k_{m} x_{n}\right)-X_{m} \cos \left(k_{m} x_{n}\right)\right)$

where $\left(x_{n}, v_{n}\right)$ are the conjugate position and velocity (in fact momentum with mass normalized to unity) of particle $n,\left(X_{m}, Y_{m}\right)$ are the conjugate generalized coordinate and momentum of the harmonic oscillator corresponding to Langmuir wave $m, \beta_{m}=$ $\left[\partial_{\omega} \mathcal{D}\left(k_{m}, \omega_{m}\right)\right]^{-1 / 2} \simeq 1 / \sqrt{2}$, and

$$
\tilde{\varepsilon}=\omega_{\mathrm{p}} \sqrt{\frac{2 \eta}{(1+\eta) N}}
$$

is the coupling coefficient where $\eta=n_{\mathrm{b}} / n_{\mathrm{p}}=N / N^{*}$. Hamiltonian $\mathcal{H}_{\mathrm{sc}}^{N, M}$ is made up of free particle terms, harmonic oscillator terms and coupling terms. Waves and particles are described on an equal footing: they inter-act. The Hamiltonian (10) is the generalization to $M>1$ waves [24, 25] of the self-consistent dynamics introduced with $M=1$ for describing the saturation of the cold beam-plasma instability by Onishchenko et al. [49] and O'Neil et al. [48]. It was recast in a Hamiltonian form by Mynick and Kaufman [47] and derived using symplectic formalism by Tennyson et al. [53]. Finally, the Hamiltonian (10) was derived directly for any $M$ from a mechanical description of the plasma as an $N$-body system [2]. This Hamiltonian (10) generates the evolution 
equations

$$
\begin{aligned}
& \dot{x}_{n}=v_{n}, \\
& \dot{v}_{n}=\tilde{\varepsilon} \operatorname{Re}\left(\sum_{m=1}^{M} \mathrm{i} \beta_{m} Z_{m} \mathrm{e}^{\mathrm{i} k_{m} x_{n}}\right), \\
& \dot{Z}_{n}=-\mathrm{i} \omega_{m} Z_{m}+\mathrm{i} \tilde{\varepsilon} \frac{\beta_{m}}{k_{m}} \sum_{n=1}^{N} \mathrm{e}^{-\mathrm{i} k_{m} x_{n}},
\end{aligned}
$$

where the dot denotes time derivative, Re denotes the real part and $Z_{m}=X_{m}+\mathrm{i} Y_{m}$. Eq. (12) makes clear the link between $Z_{m}$ and the electric field of wave $m$.

In order to keep some symmetry in the description of waves and particles, but getting rid of granularity effects due to the discrete description of particles (like spontaneous emission of waves by particles which can add difficulty from the numerical point of view [15], non commutativity of infinite time and infinite number of particles limits [33]), it is easier to work with the so-called Vlasov-wave model which is obtained as a mean-field limit (large- $N$ limit) of the dynamics defined by the Hamiltonian $\mathcal{H}_{\mathrm{sc}}^{N, M}$ $[34,22]$ in analogy to that performed by Neunzert, Dobrushin, Spohn and others for their elegant and short derivation of the Vlasov equation [52]. If we define the Radon measure (also called the empirical measure) $\sigma_{t}^{N}$ as

$$
\sigma_{t}^{N}(\mathrm{~d} x \mathrm{~d} v)=\frac{L}{N} \sum_{n=1}^{N} \delta\left(x-x_{n}(t)\right) \otimes \delta\left(v-v_{n}(t)\right) \mathrm{d} x \mathrm{~d} v,
$$

and introduce the change of variable

$$
\zeta_{m}=Z_{m} \mathrm{e}^{\mathrm{i} \omega_{m} t} N^{-1 / 2}
$$

where $\left(x_{n}(t), v_{n}(t), Z_{n}(t)\right)$ is the solution of the ordinary differential system (11)-(13) then it can be shown $[34,22]$ that the measure $\sigma_{t}^{N}$ converges weakly (for the topology induced by the dual bounded-Lipschitz distance) as $N \rightarrow \infty$ to an absolutely continuous measure $\mu_{t}:=\sigma_{t}^{\infty}$ with density $f(t, x, v)$ provided it converges at $t=0$, and that the couple $\left(f,\left\{\zeta_{m}\right\}\right)$ satisfies the following Vlasov-wave (partial differential) equations

$$
\partial_{t} f+v \partial_{x} f+\varepsilon \operatorname{Re}\left(\mathrm{i} \sum_{m=1}^{M} \beta_{m} \zeta_{m} \mathrm{e}^{\mathrm{i}\left(k_{m} x-\omega_{m} t\right)}\right) \partial_{v} f=0
$$

and

$$
\dot{\zeta}_{m}=\mathrm{i} \varepsilon \frac{\beta_{m}}{k_{m}} \frac{1}{L} \int_{\Lambda} \mathrm{e}^{-\mathrm{i}\left(k_{m} x-\omega_{m} t\right)} f(t, x, v) \mathrm{d} v \mathrm{~d} x,
$$

where $\varepsilon=\sqrt{2 \eta /(1+\eta)}$, with $\omega_{\mathrm{p}}$ normalized to unity and $\Lambda=[0, L] \times \mathbb{R}$.

System (15)-(16) preserves three constants of motion, namely the number of particles, hence the normalization of $f$ (chosen such that $\int f(t, x, v) \mathrm{d} v=\mathcal{O}(1)$ for a typical $x$ )

$$
\int_{\Lambda} f(t, x, v) \mathrm{d} v \mathrm{~d} x=L
$$


the rescaled total momentum

$$
\int_{\Lambda} v f(t, x, v) \mathrm{d} v \mathrm{~d} x+L \sum_{m=1}^{M} k_{m} \frac{\left|\zeta_{m}\right|^{2}}{2}=L \mathfrak{P}
$$

and the rescaled total energy

$$
\int_{\Lambda}\left(\frac{v^{2}}{2}-\varepsilon \operatorname{Re}\left(\sum_{m=1}^{M} \frac{\beta_{m}}{k_{m}} \zeta_{m} \mathrm{e}^{\mathrm{i}\left(k_{m} x-\omega_{m} t\right)}\right)\right) f(t, x, v) \mathrm{d} v \mathrm{~d} x+L \sum_{m=1}^{M} \omega_{m} \frac{\left|\zeta_{m}\right|^{2}}{2}=L \mathfrak{H} .
$$

Beside these "mechanical" constants of the motion, the Vlasov-wave system preserves the usual integral (Casimir) invariants of Vlasov equations like

$$
\mathcal{C}_{\Theta}[f]=L^{-1} \int_{\Lambda} \Theta(f(t, x, v)) \mathrm{d} v \mathrm{~d} x
$$

for any regular enough function $\Theta:[0,+\infty[\rightarrow \mathbb{R}$. In contrast to (17)-(19), the Casimir invariants do not exist for the singular measures $\sigma^{N}$; their conservation reflects the fact that, for any wave field $(\zeta)$, the dynamics (11)-(12) defines an area-preserving flow in $(x, v)$ space.

In the dense wave spectrum limit, the power spectrum $\psi$ of the waves, as in (2), appears through $\psi\left(v_{m}\right) \Delta v_{m}=k_{m}\left|\zeta_{m}\right|^{2} / 2$, where $\Delta v_{m}$ is the difference in phase velocity between wave $m$ and its first neighbour. Thus

$$
\sum_{\left\{m \mid u_{0} \leq v_{m}<u_{1}\right\}} k_{m}\left|\zeta_{m}\right|^{2} / 2 \simeq \int_{u_{0}}^{u_{1}} \psi(v) \mathrm{d} v
$$

for any $u_{0}, u_{1}$. We then define the waves total momentum $\mathfrak{P}_{\mathrm{w}}:=\sum_{m=1}^{M} k_{m}\left|\zeta_{m}\right|^{2} / 2 \simeq$ $\int_{\mathbb{R}} \psi(v) \mathrm{d} v$ and waves energy $\mathfrak{H}_{\mathrm{w}}:=\sum_{m=1}^{M} \omega_{m}\left|\zeta_{m}\right|^{2} / 2 \simeq \int_{\mathbb{R}} v \psi(v) \mathrm{d} v$.

If the dense spectrum limit is approximated by QL equations (1)-(2), these equations preserve the analogues of (17)-(19), with vanishing coupling energy, and satisfy an H-theorem at the expense of Casimir invariants [21].

\subsection{Dynamics when the distribution is a plateau}

We now consider [31] the extreme regime SNL where the dynamics defined by (15)-(16) starts at time $t=0$ with (i) a spectrum of Langmuir waves where all nearby waves are in resonance overlap and (ii) a particle velocity distribution function which is a single broad water bag with a height $f_{0}$ over a velocity range including the overlap domain. Both boundaries of the water bag are KAM tori related to the wave field. We first analyse a simplistic description of this dynamics, which will be useful to derive a more accurate one hereafter: (i) The initial condition corresponds to an almost spatially uniform plateau which is kept invariant by the dynamics; (ii) therefore there is no source term for the waves in (16), which keep constant complex amplitudes; (iii) hence the particle dynamics is the one defined by a prescribed spectrum of waves, which preserves the initial plateau. Clumps of particles may experience a strong turbulent trapping, but the distribution function is unaffected by this granular effect. 
Actually KAM tori, bounding the chaotic domain defined by a prescribed spectrum of waves, experience a sloshing motion due to the waves. This brings a small spatial modulation to the particle density which provides a source term for the Langmuir waves in (16). However, if the plateau is broad, the evolution of the wave spectrum is slow, which brings only a small change to the previous simplistic picture. This slow evolution suggests introducing an adiabatic description of the true dynamics. $\S$

To be specific, we consider the case where the waves phase velocities range over an interval $\left[u_{0}, u_{1}\right]$, the nearest KAM tori have velocities $v_{\mathrm{a}}$ and $v_{\mathrm{b}}$ and the water bag is bounded by KAM tori with velocities $\left[v_{0}, v_{1}\right]$, with $v_{0} \lesssim v_{\mathrm{a}} \lesssim u_{0}<u_{1} \lesssim v_{\mathrm{b}} \lesssim v_{1}$ (with typically $s_{\mathrm{ov}} \ll\left|u_{1}-u_{0}\right| / \Delta v_{\varphi}$ ). Therefore the plateau width at any position is about $\Delta v_{\text {plat }}=v_{1}-v_{0}$, which is essentially equal to the chaotic domain width $\Delta v_{\mathrm{KAM}}=v_{\mathrm{b}}-v_{\mathrm{a}}$ and to the wave spectrum width $\Delta v_{\mathrm{spec}}=u_{1}-u_{0}$. Moreover, assume that the wave numbers are all of comparable order of magnitude, typically $k$. Particles with velocity $v$ experience the oscillations of a wave $m$ at relative frequencies $\Omega_{m}=\omega_{m}-k_{m} v$, and for a particle or a wave with velocity $v$ the nearest KAM velocity defines $\Omega_{\min }=k \min \left(v_{\mathrm{b}}-v, v-v_{\mathrm{a}}\right)$; we denote by $\Delta v_{\text {edge }}=\min \left(v_{1}-u_{1}, u_{0}-v_{0}\right)$ the relative velocity of the typical edges of the plateau with respect to the extremal wave velocities. The resonance overlap in the wave spectrum is characterized by the small parameter $1 / s_{\text {ov }} \sim \delta \Omega /\left(k \varepsilon \zeta_{*}\right)^{1 / 2} \ll 1$, where $\delta \Omega \simeq k \Delta v_{\varphi}$ is the Doppler frequency detuning of two nearby waves and $\zeta_{*}$ is the typical modulus of a wave amplitude.

We now formalize this adiabatic description. Let $\zeta_{m}(t)$ be the value of $\zeta_{m}$ at time $t$ in the self-consistent dynamics. Consider the non self-consistent dynamics $\mathrm{D}\left(t_{0}\right)$ defined by the self-consistent spectrum of Langmuir waves frozen at time $t_{0}$, as defined by (15) where the $\zeta_{m}$ 's are substituted with the $\zeta_{m}\left(t_{0}\right)$ 's. The chaotic domain $\mathrm{C}\left(t_{0}\right)$ in singleparticle (Boltzmann or $\mu$ ) phase space $(x, v)$ defined by this frozen wave spectrum is bounded above and below in $v$ by two KAM tori, respectively $\mathrm{T}_{\mathrm{a}}\left(t_{0}\right)$ and $\mathrm{T}_{\mathrm{b}}\left(t_{0}\right)$. The initial particle distribution function $f(x, v, 0)$ is assumed to be uniform on $\mathrm{C}(0)$; let $f_{0}$ be this uniform value. During the adiabatic evolution corresponding to the true selfconsistent dynamics, $f(x, v, t)$ stays uniform on $\mathrm{C}(t)$ and keeps the value $f_{0}$. We are left with the calculation of the modulation of the width of a single water bag with height $f_{0}$. The modulation of this width is given by that of KAM tori $\mathrm{T}_{\mathrm{a}}\left(t_{0}\right)$ and $\mathrm{T}_{\mathrm{b}}\left(t_{0}\right)$, which may be computed by perturbation theory in the typical amplitude $\zeta_{*}$ of the Langmuir waves.

Since mode-mode coupling is a four-wave process, the first nonvanishing contribution to (16) is of order $\zeta_{*}^{3}$; we estimate it in Appendix B. For waves with phase velocities further than $\mathrm{O}\left(\Delta v_{\text {edge }}\right)$ from the edges of the plateau this

$\S$ These KAM tori are associated with a prescribed spectrum of waves, for which each particle evolves under a 1.5 degrees of freedom hamiltonian (9). For the self-consistent evolution of waves and particles in the $N+M \gg 1$ degrees of freedom dynamics of (10), phase space is $2(N+M)$-dimensional and near-integrable behaviour is described by Nekhoroshev's theorem. The KAM tori no longer bound the motion on large scales, but Arnold diffusion may occur on long time scales. Note that even the "familiar" Landau damping (in its nonlinear guise) is akin to a weak KAM behaviour in the infinite dimensional phase space of the Vlasov-Poisson partial differential equation [45, 46]. 
dominant contribution to $\dot{\zeta}_{m}(t)$ scales like a sum of $\varepsilon^{4} \beta^{4}|\zeta|^{3} k /\left[\left(v_{1}-v_{0}\right) \Omega_{\text {min }}^{4}\right]$ and $\varepsilon^{4} \beta^{4}|\zeta|^{3} /\left(8 v_{1}\left(v_{1}-v_{0}\right) \Omega_{\min }^{2} \delta \Omega\right)$. Therefore, in the limit of a broad plateau $\left(\Delta v_{\text {plat }} \rightarrow \infty\right)$ with a fixed discretization $\delta \Omega, \dot{\zeta}_{m}$ will vanish when we consider waves in a central range. If we consider waves near the extremal phase velocity in a moderately wider plateau, the edges must still be $\Omega_{\min } / k$ away from these waves, which scales at worst like $\left(\varepsilon \beta \zeta_{*}\right)^{2 / 3}$, which still leaves terms not worse than $\zeta_{*}^{5 / 3} / \delta \Omega$ and $\zeta_{*}^{1 / 3}$. In the seemingly more dangerous limit $\delta \Omega \rightarrow 0$, the wave evolutions will not depart from adiabaticity before the time scale $\delta \Omega^{-1} \sim \tau_{\text {discr }}$, which tends to infinity. This justifies a posteriori our previous adiabatic approximation for all waves.

As a result the plateau dynamics (further than $\Omega_{\min } / k$ from its boundaries) is almost the same as in a prescribed field of Langmuir waves. Therefore the chaotic motion of particles is almost unchanged due to the nonlinear coupling of Langmuir waves.

\subsection{Inconsistency of several models}

We have just shown that self-consistency vanishes in the plateau regime of the bumpon-tail instability if the plateau is broad enough, because the particle transport only rearranges particles without changing $f$ itself within the plateau, depriving waves from this source. This means that the diffusion coefficient $D(v)$ of particles with momentum $v$ is that found for the dynamics of particles in a prescribed spectrum of Langmuir waves. Let $D_{\mathrm{QL}}(v)$ be the quasilinear value of this coefficient. In the resonance overlap regime $D / D_{\mathrm{QL}}$ may cover a large range of values $[13,21,20]$. In particular $D \simeq D_{\mathrm{QL}}$ is obtained for random phases of the waves and strong resonance overlap [13, 21, 20, 23]. We stress that strong resonance overlap alone is not enough to provide $D \simeq D_{\mathrm{QL}}$.

Let $\gamma_{\mathrm{L}}$ be the maximum Landau growth rate of Langmuir waves for the instantaneous value of $f$. As $\tau_{\text {spread }} \simeq 4\left(k^{2} D\right)^{-1 / 3}$ is the time after which the ballistic approximation fails for particles diffusing with the maximum instantaneous $D$ due to a spectrum of Langmuir waves with typical wavenumber $k$, the plateau regime corresponds to $\gamma_{\mathrm{L}} \tau_{\text {spread }}=0$. Since $D / D_{\mathrm{QL}}$ may cover a large range of values in this regime, $\gamma_{\mathrm{L}} \tau_{\text {spread }} \ll 1$ does not imply per se any renormalization or non-renormalization of $D / D_{\mathrm{QL}}$, nor of $\gamma / \gamma_{\mathrm{L}}$ by wave-particle momentum conservation. This contradicts previous works using $\gamma_{\mathrm{L}} \tau_{\text {spread }} \ll 1$ to try and prove the validity of quasilinear theory [42, 43, 28, 21] and the "turbulent trapping" Ansatz aiming at the contrary [40]. The value of $D / D_{\mathrm{QL}}$ in the plateau regime of the bump-on-tail instability depends on the kind of wave spectrum the beam-plasma system reaches during the regime INL of the instability, and not only on condition $\gamma_{\mathrm{L}} \tau_{\text {spread }} \ll 1$, as assumed by these works. The following numerical simulation using the Vlasov-wave model attempts to uncover this spectrum. 


\section{Numerical approximation of the Vlasov-wave equation}

Note that the system (15)-(16) admits a unique global regular classical solution, since it is easy to show that the force field is regular for any finite $M$. Therefore the use of high-order numerical scheme to approximate the system (15)-(16) is relevant.

In this section we describe the scheme for the numerical approximation of the above introduced Vlasov-wave model (15)-(16). Here we use a semi-Lagrangian scheme with an $(x, v)$-area-preserving integrator in time obtained by decomposing the dynamics in integrable Hamiltonian steps. A natural and simple choice is the Strang time-splitting strategy also known as the centred leapfrog symplectic integrator. Roughly speaking, this intensively used scheme (see $[14,32,7,8,9,10]$ and references therein) consists in splitting the full transport operator into two easily integrable transport operators one in the physical space, the second one in the velocity or momentum space - and solving them successively in a right order to get high-order approximation in time of the complete transport operator. Moreover $B$-spline interpolation of high order is used to reconstruct the distribution function on the $(x, v)$-space mesh and interpolate its values at the origins of the characteristic curves set. Such a Eulerian algorithm, usually named Vlasov code, has the advantages to provide an excellent resolution all over the $(x, v)$ space including very low density regions, since convergence and a priori high-order accuracy properties of these schemes are well controlled and understood. In addition, conservation of the constants of motion can be ensured with a good accuracy by semi-Lagrangian schemes. Recently, mathematical proofs of convergence and a priori high-order error estimates (very high-order accuracy) of these schemes were obtained in a series of papers $[7,8,9,3]$.

Let $\mathcal{M}_{h}$ be a discretization of the $\mu$-space $(x, v)$, with $N_{x} \times N_{v}$ mesh points with steps $\Delta x$ and $\Delta v$, and $f_{h}^{n}$ an approximation of $f$ at time $t^{n}$ on $\mathcal{M}_{h}$. The general algorithm to compute $f_{h}^{n+1}$ for $t^{n+1}=t^{n}+\Delta t$ consists in three steps.

1) Half time advection in physical space. This step consists in solving the equation

$$
\partial_{t} f+v \partial_{x} f=0, \quad t \in\left[t^{n}, t^{n+1 / 2}\right], \quad \text { with } f\left(t^{n}\right)=f_{h}^{n} .
$$

To solve (21) we integrate its associated characteristic curves equation

$$
\frac{\mathrm{d} X}{\mathrm{~d} t}(t)=V(t)
$$

on the time interval $\left[t^{n}, t^{n+1 / 2}\right]$ with $t^{n+1 / 2}=t^{n}+\Delta t / 2$. We then get

$$
X^{n}-X^{n+1 / 2}=\int_{t^{n+1 / 2}}^{t^{n}} V(t) \mathrm{d} t=\int_{t^{n+1 / 2}}^{t^{n}} V\left(t^{n}\right) \mathrm{d} t+\mathcal{O}\left(\Delta t^{2}\right) \simeq-V^{n} \Delta t / 2,
$$

where $\left(X^{n+1 / 2}, V^{n}\right) \in \mathcal{M}_{h}$ and $X^{n}$ is the origin of the characteristic curve we look for. Therefore the new distribution function $f_{h}^{\star}$ is such that

$$
f_{h}^{\star}(x, v):=\tilde{f}_{h}^{n+1 / 2}\left(X^{n+1 / 2}, V^{n}\right)=f_{h}^{n}\left(X^{n}, V^{n}\right), \quad \forall(x, v) \in \mathcal{M}_{h} .
$$

As $\left(X^{n}, V^{n}\right)$ is generally not in $\mathcal{M}_{h}$, it is interpolated using a $B$-spline. 
2) Time advection in velocity space. This step consists in solving the equation

$$
\partial_{t} f+F\left(t, x,\left\{\zeta_{m}\right\}\right) \partial_{v} f=0, \quad t \in\left[t^{n}, t^{n+1}\right], \quad \text { with } f\left(t^{n}\right)=f_{h}^{\star},
$$

where $F$ is the factor of $\partial_{v} f$ in (15). To solve (22) we integrate its characteristics equation (12), viz.

$$
\frac{\mathrm{d} V}{\mathrm{~d} t}(t)=F\left(t, X(t),\left\{\zeta_{m}(t)\right\}\right)
$$

on the time interval $\left[t^{n}, t^{n+1}\right]$. We then get

$$
\begin{aligned}
V^{n+1}-V^{n} & =\int_{t^{n}}^{t^{n+1}} F\left(t, X(t),\left\{\zeta_{m}(t)\right\}\right) \mathrm{d} t \\
& =\int_{t^{n}}^{t^{n+1}} F\left(t^{n+1 / 2}, X\left(t^{n+1 / 2}\right),\left\{\zeta_{m}\left(t^{n+1 / 2}\right)\right\}\right) \mathrm{d} t+\mathcal{O}\left(\Delta t^{3}\right) \\
& \simeq F\left(t^{n+1 / 2}, X^{n+1 / 2},\left\{\zeta_{m}^{n+1 / 2}\right\}\right) \Delta t,
\end{aligned}
$$

where $\left(X^{n+1 / 2}, V^{n+1}\right) \in \mathcal{M}_{h}$ and $V^{n}$ is the origin of the characteristic curve we look for. Therefore the new distribution function $f_{h}^{\star \star}$ is such that

$$
f_{h}^{\star \star}(x, v):=\hat{f}_{h}^{n+1 / 2}\left(X^{n+1 / 2}, V^{n+1}\right)=f_{h}^{\star}\left(X^{n+1 / 2}, V^{n}\right), \quad \forall(x, v) \in \mathcal{M}_{h} .
$$

There remains to compute a "good" approximation of $\left\{\zeta_{m}^{n+1 / 2}\right\}$, consistent with this step. To this purpose we integrate the wave equation (16) on the time interval $\left[t^{n}, t^{n+1}\right]$ and, using mid-point quadrature rule $\|$, we obtain for all $m \in[1, M]$ :

$$
\begin{aligned}
\zeta_{m}^{n+1 / 2}-\zeta_{m}^{n-1 / 2} & :=\zeta_{m}\left(t^{n+1 / 2}\right)-\zeta_{m}\left(t^{n-1 / 2}\right) \\
& =\mathrm{i} \varepsilon \frac{\beta_{m}}{k_{m}} \frac{1}{L} \int_{t^{n-1 / 2}}^{t^{n+1 / 2}} \int_{\Lambda} \mathrm{e}^{-\mathrm{i}\left(k_{m} x-\omega_{m} t\right)} f(t, x, v) \mathrm{d} v \mathrm{~d} x \mathrm{~d} t \\
& \simeq \mathrm{i} \varepsilon \frac{\beta_{m}}{k_{m}} \Delta t \Delta v \frac{\Delta x}{L} \mathrm{e}^{\mathrm{i} \omega_{m} t^{n}} \sum_{q=1}^{N_{x}} \sum_{p=1}^{N_{v}} f\left(t^{n}, x_{q}, v_{p}\right) \mathrm{e}^{-\mathrm{i} k_{m} x_{q}}+\mathcal{O}\left(\Delta t^{3}\right) \\
& \simeq \mathrm{i} \varepsilon \frac{\beta_{m}}{k_{m}} \Delta t \Delta v \frac{\Delta x}{L} \mathrm{e}^{\mathrm{i} \omega_{m} t^{n}} \sum_{q=1}^{N_{x}} \sum_{p=1}^{N_{v}} f_{h}^{n}\left(x_{q}, v_{p}\right) \mathrm{e}^{-\mathrm{i} k_{m} x_{q}} \\
& \simeq \mathrm{i} \varepsilon \frac{\beta_{m}}{k_{m}} \Delta t e^{\mathrm{i} \omega_{m} t^{n}} \frac{\Delta x}{L} \sum_{q=1}^{N_{x}} \rho_{h}^{n}\left(x_{q}\right) \mathrm{e}^{-\mathrm{i} k_{m} x_{q}} \\
& \simeq \mathrm{i} \varepsilon \frac{\beta_{m}}{k_{m}} \Delta t \mathrm{e}^{\mathrm{i} \omega_{m} t^{n}} \mathcal{F}_{h}\left(\rho_{h}^{n}, k_{m}\right),
\end{aligned}
$$

where $\rho_{h}^{n}(\cdot)=\sum_{p=1}^{N_{v}} f_{h}^{n}\left(\cdot, v_{p}\right) \Delta v$ and $\mathcal{F}_{h}(\cdot, k)$ denotes the discrete Fourier transform at wave number $k$.

3) Half time advection in physical space. This step (formally identical to step 1) solves the equation

$$
\partial_{t} f+v \partial_{x} f=0 \quad t \in\left[t^{n+1 / 2}, t^{n+1}\right], \quad \text { with } f\left(t^{n+1 / 2}\right)=f_{h}^{\star \star},
$$

\|It is accurate to second order and a good approximation to a more expensive three-substeps fully symplectic integrator which would also require splitting (23) in three corresponding substeps. 
by convecting $f$ along the characteristic, using

$$
X^{n+1}-X^{n+1 / 2}=\int_{t^{n+1 / 2}}^{t^{n+1}} V(t) \mathrm{d} t=\int_{t^{n+1 / 2}}^{t^{n+1}} V\left(t^{n+1}\right) \mathrm{d} t+\mathcal{O}\left(\Delta t^{2}\right) \simeq V^{n+1} \Delta t / 2,
$$

where $\left(X^{n+1}, V^{n+1}\right) \in \mathcal{M}_{h}$ and $X^{n+1 / 2}$ is the origin of the characteristic curve we look for. Therefore the new distribution function is such that

$$
f_{h}^{n+1}(x, v)=f_{h}^{n+1}\left(X^{n+1}, V^{n+1}\right)=f_{h}^{\star \star}\left(X^{n+1 / 2}, V^{n+1}\right), \quad \forall(x, v) \in \mathcal{M}_{h} .
$$

Putting together steps 1 and 3 shows that the time-discretized dynamics works in the classical leap-frog way where velocity is advanced at times $t^{n}$, while the position is advanced at times $t^{n+1 / 2}$. The algorithm is reversible within the accuracy of the spline interpolation (which prevents step 3 from being exactly the adjoint of step 1).

\section{Self-consistent simulations parameters}

In the next sections we present self-consistent simulations of the Vlasov-wave system (15)-(16). In order to construct relevant test cases to assess the validity of quasilinear theory we must estimate some physical parameters. Moreover, since the plateau formation in the strong chaotic regime could take a very long time, we need to optimize the values of the physical and numerical parameters; hence a priori estimates for these parameters are required. They are based on momentum conservation since we can clearly separate the two contributions of the waves and particles. In contrast, the use of energy conservation is not appropriate because it involves a third term, associated with the wave-particle coupling, which is more difficult to estimate a priori.

Since $k_{m} \lambda_{\mathrm{D}} \ll 1$, using the Bohm-Gross relation we deduce that $\omega_{m} \simeq \omega_{\mathrm{p}}=1$. Therefore the phase velocity range of interest will be $v_{\varphi} \in\left[u_{0}, u_{1}\right]=\left[k_{\max }^{-1}, k_{\min }^{-1}\right]$, with a width $\Delta v_{\text {spec }}=u_{1}-u_{0}$. From the $(x, v)$-area conservation and the normalization of the distribution function, the height of the particle velocity distribution function plateau in the long-time asymptotic regime is approximately $\left(u_{1}-u_{0}\right)^{-1}$ (to first approximation, neglecting the particles which might be outside the wave velocity range as they are weakly perturbed by the waves). We assume

$$
k_{m}=\frac{2 \pi}{L}\left(\nu_{0}+m\right), \quad \text { with } m \in[1, M],
$$

so that $v_{\varphi, m}=\omega_{\mathrm{p}} / k_{m}=1 / k_{m}$ which implies

$$
\begin{aligned}
& u_{0} \quad=\frac{\nu_{0}+1}{M-1} \Delta v_{\mathrm{spec}} \simeq \frac{\nu_{0}}{M} \Delta v_{\mathrm{spec}} \\
& u_{1} \quad=\frac{L}{2 \pi\left(\nu_{0}+1\right)} \simeq\left(1+\frac{\nu_{0}}{M}\right) \Delta v_{\mathrm{spec}} \\
& L \quad=2 \pi\left(\nu_{0}+M\right) u_{0} \simeq 2 \pi\left(1+\frac{\nu_{0}}{M}\right) \nu_{0} \Delta v_{\mathrm{spec}}, \\
& \frac{2 \pi M}{L}=\left(1+\frac{\nu_{0}}{M}\right)^{-1} u_{0}^{-1} \simeq\left(\left(1+\frac{\nu_{0}}{M}\right) \frac{\nu_{0}}{M} \Delta v_{\mathrm{spec}}\right)^{-1}
\end{aligned}
$$


where we assumed $\nu_{0} \gg 1$ and $M \gg 1$. Using momentum conservation (18), assuming that the initial (resp. final) distribution function $f_{0}$ (resp. $f_{\infty}$ ) is homogeneous in space, and neglecting $\psi(0, v)$ we obtain

$$
\begin{aligned}
\mathfrak{P}_{\mathrm{w}}^{\infty}-\mathfrak{P}_{\mathrm{w}}^{0} & =\int_{u_{0}}^{u_{1}} v\left(f_{0}-f_{\infty}\right) \mathrm{d} v=\int_{u_{0}}^{u_{1}} \psi(\infty, v) \mathrm{d} v \\
& =\sum_{m=1}^{M} \psi\left(\infty, v_{m}\right) \Delta v_{m}=\sum_{m=1}^{M} k_{m} \frac{\left|\zeta_{m}(\infty)\right|^{2}}{2} \\
& =\int_{k_{\min }}^{k_{\max }} \Psi(\infty, k) \mathrm{d} k=\sum_{m=1}^{M} \Psi\left(\infty, k_{m}\right) \Delta k_{m}
\end{aligned}
$$

where we used (20) and $\Psi(t, k)=\psi(t, v)|\mathrm{d} v / \mathrm{d} k|$ to express the power spectrum of waves with respect to wavenumber. Equation (23) can also be obtained by integrating the conservation law

$$
\partial_{t} \nu(t, v)=0
$$

with

$$
\nu(t, v)=f(t, v)-\partial_{v} \psi(t, v)
$$

(which follows [21] from QL equations (1)-(4)) with the initial condition $\partial_{v} \psi(0, v)=0$ and the boundary condition $\psi\left(t, u_{0}\right)=\psi\left(t, u_{1}\right)=0$.

\subsection{Estimates of physical parameters}

We first estimate a mean value (denoted by $\langle a\rangle=M^{-1} \sum_{m} a_{m}$ for any variable $a$ ) of the wave power spectrum at the final time where the plateau regime is well settled. Since $\Delta k_{m}=k_{m+1}-k_{m}=2 \pi / L$, we have $\mathfrak{P}_{\mathrm{w}}^{\infty}-\mathfrak{P}_{\mathrm{w}}^{0} \simeq\left(1+\frac{\nu_{0}}{M}\right)^{-1} \nu_{0}^{-1} \Delta v_{\text {spec }}^{-1} \sum_{m=1}^{M} \Psi\left(\infty, k_{m}\right)$ and thus

$$
\langle\Psi(\infty)\rangle \simeq\left(1+\frac{\nu_{0}}{M}\right) \frac{\nu_{0}}{M} \Delta v_{\mathrm{spec}}\left(\mathfrak{P}_{\mathrm{w}}^{\infty}-\mathfrak{P}_{\mathrm{w}}^{0}\right) .
$$

Since $D_{\mathrm{QL}}\left(t, v_{m}\right) \simeq \pi \eta \beta_{m}^{2}\left|\zeta_{m}\right|^{2}\left(k_{m} \Delta v_{m}\right)^{-1}=2 \pi \eta \beta_{m}^{2} k_{m}^{-2} \psi\left(t, v_{m}\right) \simeq \pi \eta \Psi\left(t, k_{m}\right)$, using (25) we obtain for the QL diffusion coefficient

$$
\left\langle D_{\mathrm{QL}}(\infty)\right\rangle \simeq \pi \eta\left(1+\frac{\nu_{0}}{M}\right) \frac{\nu_{0}}{M} \Delta v_{\mathrm{spec}}\left(\mathfrak{P}_{\mathrm{w}}^{\infty}-\mathfrak{P}_{\mathrm{w}}^{0}\right)
$$

which determines the characteristic time $\tau_{\mathrm{D}}(6)$.

Then we estimate the Kubo-like number. As $K_{\mathrm{D}, m}^{-1}$ estimates the number of velocity diffusion widths through the wave spectrum at saturation, we shall test the condition $K_{\mathrm{D}, m}^{-1} \gg 1$. Using (26) obtains

$$
K_{\mathrm{D}}^{-1} \simeq\left\langle K_{\mathrm{D}, m}^{-1}\right\rangle=\Delta v_{\mathrm{spec}}\left\langle\left(k_{m} / D_{\mathrm{QL}, m}\right)^{1 / 3}\right\rangle \simeq \Delta v_{\mathrm{spec}}\left\langle k_{m}^{1 / 3}\right\rangle\left\langle D_{\mathrm{QL}, m}\right\rangle^{-1 / 3},
$$

where, for $\alpha \neq-1$,

$$
\begin{aligned}
\left\langle k_{m}^{\alpha}\right\rangle & \simeq \frac{L}{2 \pi M} \int_{k_{\min }}^{k_{\max }} k^{\alpha} \mathrm{d} k=\frac{L}{2 \pi M} \frac{k_{\max }^{\alpha+1}-k_{\min }^{\alpha+1}}{\alpha+1} \\
& \simeq \frac{L}{2(1+\alpha) \pi M \Delta v_{\mathrm{spec}}}\left(\left(\frac{\nu_{0}}{M}\right)^{-(\alpha+1)}-\left(1+\frac{\nu_{0}}{M}\right)^{-(\alpha+1)}\right) .
\end{aligned}
$$


We also estimate the parameter $\mathcal{B}^{-1}$. From $\Delta v_{\varphi, m} \simeq 2 \pi /\left(L k_{m}^{2}\right)$ (as $k_{m}$ varies linearly with $m$ ) we obtain $\mathcal{B}_{m}^{-1}=\Delta v_{\mathrm{D}, m} /\left(\Delta v_{\varphi, m}\right)=D_{\mathrm{QL}, m}^{1 / 3} k_{m}^{5 / 3} L /(2 \pi)$ and

$$
\mathcal{B}^{-1} \simeq\left\langle\mathcal{B}_{m}^{-1}\right\rangle=\frac{L}{2 \pi}\left\langle k_{m}^{5 / 3} D_{\mathrm{QL}, m}^{1 / 3}\right\rangle \simeq \frac{L}{2 \pi}\left\langle D_{\mathrm{QL}, m}\right\rangle^{1 / 3}\left\langle k_{m}^{5 / 3}\right\rangle .
$$

The parameter $\mathcal{B}_{m}$ (relevant to the strong overlap regime of many waves) is related to the more familiar Chirikov resonance overlap parameter $s_{\text {ov }, m}=(512 / \pi)^{1 / 4} \mathcal{B}_{m}^{-3 / 4} \simeq 3.6 \mathcal{B}_{m}^{-3 / 4}$ (more appropriate for few waves overlapping moderately). Again,

$$
s_{\mathrm{ov}}=\left\langle s_{\mathrm{ov} m}\right\rangle \simeq 8 \sqrt{\pi} L^{-3 / 4}\left\langle D_{\mathrm{QL}, m}\right\rangle^{-1 / 4}\left\langle k_{m}^{-5 / 4}\right\rangle .
$$

Finally note that $\mu \ll 1$ at the beginning of the instability and $\mu \gg 1$ in the saturation phase. To ensure that $\mu \ll 1$ at the beginning, it is equivalent to guarantee that we have an initial linear regime where wave $m$ grows exponentially with the Landau growth rate

$$
\gamma_{\mathrm{L}, m}=\gamma_{\mathrm{L}}\left(0, v_{m}\right)=\frac{\pi}{2} \frac{\eta}{1+\eta} v_{m}^{2} \partial_{v} f_{0}\left(v_{m}\right) .
$$

In order to ensure that $\mu \gg 1$ at the end of the simulation we verify that the plateau regime settled in, with a final distribution function $f$ almost constant in velocity. Although these estimates are crude $(\langle a b\rangle \sim\langle a\rangle\langle b\rangle)$, they capture the relevant scaling powers for the subsequent discussion.

\subsection{Estimates of numerical parameters}

We now estimate the numerical parameters of the simulations. Our mesh $\mathcal{M}_{h}$ is a regular cartesian grid spanning $[0, L] \times\left[v_{\min }, v_{\max }\right]$. Boundary conditions are periodic in space $x$, while the range $\left[v_{\min }, v_{\max }\right]$ must contain both the whole spectrum of wave phase velocities $\left[u_{0}, u_{1}\right]$ and the support of the particle distribution function during the whole simulation. Thus $v_{\min }<u_{0}<u_{1}<v_{\max }$ in such a way that $f\left(t, x, v_{\min }\right)=f\left(t, x, v_{\max }\right)=$ 0 for all $(t, x)$. In terms of section 2.2 we thus need $v_{\min }<v_{0}<v_{1}<v_{\max }$. We might estimate $v_{\text {min }} \leq u_{0}-n_{\mathrm{KAM}} s_{\mathrm{ov}} \Delta v_{\varphi}$ and $v_{\max } \geq u_{1}+n_{\mathrm{KAM}} s_{\mathrm{ov}} \Delta v_{\varphi}$ for some real positive $n_{\mathrm{KAM}}>2$.

Let us define $\Delta x$ (resp. $\Delta v$ ) as the space discretization step (resp. the velocity discretization step) for the mesh $\mathcal{M}_{h}$. We set $\Delta x$ as the ratio of the shortest length $2 \pi / k_{\max }$ we need to resolve to $n_{\delta x}$ with $n_{\delta x} \geq 1$ a positive real number. Similarly we set $\Delta v$ as the ratio of a typical phase velocity difference $\Delta v_{\varphi}=\left\langle\Delta v_{\varphi, m}\right\rangle \simeq \Delta v_{\text {spec }} / M$ to $n_{\Delta v_{\varphi}}$ with $n_{\Delta v_{\varphi}} \geq 1$ a positive real number. So we obtain

$$
\Delta x=\frac{2 \pi}{n_{\delta x} k_{\max }} \simeq \frac{2 \pi}{n_{\delta x}} \frac{\nu_{0}}{M} \Delta v_{\mathrm{spec}}, \quad N_{x}=\frac{L}{\Delta x} \simeq n_{\delta x}\left(\nu_{0}+M\right),
$$

and

$$
\Delta v=\frac{\Delta v_{\varphi}}{n_{\Delta v_{\varphi}}} \simeq \frac{\Delta v_{\text {spec }}}{M n_{\Delta v_{\varphi}}}, \quad N_{v}=\frac{\Delta v_{\text {spec }}}{\Delta v} \simeq n_{\Delta v_{\varphi}} M
$$

Next we estimate the time step $\Delta t$. There are two ways for estimating this parameter and we will see that they give equivalent results. From purely numerical 
considerations we can estimate $\Delta t$ by setting the Courant-Friedrichs-Lewy (CFL)-like condition $\Delta x \sim v_{\max } \Delta t$. By taking $v_{\max }=u_{1}$ we obtain

$$
\Delta t=u_{1}^{-1} \Delta x \simeq \frac{2 \pi}{n_{\delta x}} \frac{\nu_{0}}{M}\left(1+\frac{\nu_{0}}{M}\right)^{-1} .
$$

We must stress that semi-Lagrangian schemes are unconditionally stable and therefore no CFL condition is required to ensure the stability of the scheme. Nevertheless, in order to guarantee accurate numerical results, in practice we have to ensure that the numerical and physical velocity of waves are of the same order, which leads to the CFL-like condition (27).

From physical considerations the time step $\Delta t$ must be chosen such that phase $\Phi_{m}=k_{m} x-\omega_{m} t$ of wave $m$ does not vary too much over time $\Delta t$. In practice this condition is required so that the discretized force field which appears in the Vlasov-wave equations (15)-(16) is sufficiently well sampled. Therefore we must have $\max \Delta \Phi_{m}=2 \pi / n_{\Phi}$ with $n_{\Phi} \geq 1$ a positive real number, with

$$
\begin{aligned}
\max \left|\Delta \Phi_{m}\right| & =\Delta t \max \left|k_{m} \frac{\Delta x}{\Delta t}-\omega_{m}\right|=\Delta t \max \left|k_{m} v-\omega_{m}\right| \\
& =\Delta t\left|k_{\max } v_{\max }-1\right|=\Delta t\left|k_{\max } u_{1}-1\right| \simeq \Delta t\left(\frac{\nu_{0}}{M}\right)^{-1} .
\end{aligned}
$$

As a consequence we get

$$
\Delta t \simeq \frac{2 \pi}{n_{\Phi}} \frac{\nu_{0}}{M}
$$

The time step estimates (27) and (28) are equivalent provided that $\nu_{0} / M \ll 1$, which will be the case. Finally we can estimate the final time $T_{\text {end }}$ as

$$
T_{\text {end }} \propto \max \left\{\left\langle\gamma_{\mathrm{L}}(0)\right\rangle^{-1}, T_{\text {dif }}\right\},
$$

where

$$
T_{\text {dif }}=\frac{\left\langle\Delta v_{\text {spec }}^{2}\right\rangle}{2\left\langle D_{\mathrm{QL}}(\infty)\right\rangle} \simeq \Delta v_{\mathrm{spec}}\left(2 \pi \eta\left(1+\frac{\nu_{0}}{M}\right) \frac{\nu_{0}}{M}\left(\mathfrak{P}_{\mathrm{w}}^{\infty}-\mathfrak{P}_{\mathrm{w}}^{0}\right)\right)^{-1}
$$

while we take

$$
\left\langle\gamma_{\mathrm{L}}(0)\right\rangle=\frac{\pi}{2} \frac{\eta}{1+\eta}\left(u_{1}-u_{0}\right)^{-1} \int_{u_{0}}^{u_{1}} v^{2} \partial_{v} f_{0}(v) \mathrm{d} v \simeq \frac{\pi}{3} \frac{\eta}{1+\eta} \frac{u_{1}^{3}-u_{0}^{3}}{\left(u_{1}-u_{0}\right)^{3}},
$$

for an initial triangular distribution function, and $\left\langle\gamma_{\mathrm{L}}(0)\right\rangle$ as a prescribed constant for an initial hyperbolic distribution function (see the next sections). The number of time iterations is set to $N_{T}=T_{\text {end }} / \Delta t$.

\subsection{Asymptotic estimates for all the parameters}

Finally we find the asymptotic expression for the parameters defined in sections 4.1 and 4.2 when we consider $\nu_{0} / M \ll 1$ and $u_{1}=1$ which implies $\Delta v_{\text {spec }}=\left(1+\frac{\nu_{0}}{M}\right)^{-1} \simeq 1$. We obtain (up to factors close to unity, such as $\pi^{1 / 3}$ )

$$
\langle\Psi(\infty)\rangle \simeq\left(\frac{\nu_{0}}{M}\right)\left(\mathfrak{P}_{\mathrm{w}}^{\infty}-\mathfrak{P}_{\mathrm{w}}^{0}\right)
$$




$$
\begin{aligned}
\left\langle D_{\mathrm{QL}}(\infty)\right\rangle & \simeq \pi \eta\langle\Psi(\infty)\rangle \simeq \pi \eta\left(\frac{\nu_{0}}{M}\right)\left(\mathfrak{P}_{\mathrm{w}}^{\infty}-\mathfrak{P}_{\mathrm{w}}^{0}\right) \\
K_{\mathrm{D}}^{-1} & \simeq 1 / \Delta v_{\mathrm{D}} \simeq \eta^{-1 / 3}\left(\frac{\nu_{0}}{M}\right)^{-2 / 3}\left(\mathfrak{P}_{\mathrm{w}}^{\infty}-\mathfrak{P}_{\mathrm{w}}^{0}\right)^{-1 / 3}, \\
\mathcal{B}^{-1} & \simeq \eta^{1 / 3}\left(\frac{\nu_{0}}{M}\right)^{-4 / 3} \nu_{0}\left(\mathfrak{P}_{\mathrm{w}}^{\infty}-\mathfrak{P}_{\mathrm{w}}^{0}\right)^{1 / 3}, \\
N_{x} & \simeq\left(\frac{\nu_{0}}{M}\right)^{-1} \nu_{0} n_{\delta x}=M n_{\delta x}, \\
N_{v} & \simeq\left(\frac{\nu_{0}}{M}\right)^{-1} \nu_{0} n_{\Delta v_{\varphi}}=M n_{\Delta v_{\varphi}}, \\
\Delta t & \simeq \frac{2 \pi}{n_{\Phi}}\left(\frac{\nu_{0}}{M}\right), \\
T_{\text {end }} & \simeq \max \left\{\left\langle\gamma_{\mathrm{L}}(0)\right\rangle^{-1}, T_{\mathrm{dif}}\right\} \simeq \max \left\{\eta^{-1},\left\langle D_{\mathrm{QL}}(\infty)\right\rangle^{-1}\right\} .
\end{aligned}
$$

We first note that, given the wavenumbers $k_{m}$ and the dispersion relation, the ratio $\tau_{\text {ac }} / \tau_{\text {discr }}=\Delta v_{\varphi} / \Delta v_{\text {spec }}=1 / M$ is independent of the waves and particles evolutions, and we need a large ratio to allow observations of intermediate scales like $\tau_{\mathrm{D}}$ or $\Delta v_{\mathrm{D}}$. Constraint $\eta \ll 1$ is also desired to ensure that $\mu \ll 1$ initially, and that both $\gamma_{\mathrm{L}} / \omega_{\mathrm{p}}$ and $D_{\mathrm{QL}}$ are small when $\partial_{v} f$ and $\psi$ are of the order of unity.

From these asymptotic values we observe that conditions $\eta \ll 1$ and $\nu_{0} / M \ll 1$ work towards ensuring a small value for the Kubo number $K_{\mathrm{D}}$ and the Dupree width $\Delta v_{\mathrm{D}}$ (which means several resonance boxes in the wave velocity range, see Appendix A). On the other hand, conditions $\eta \gg 1, \nu_{0} \gg 1$ and $\nu_{0} / M \ll 1$ favour a strong resonance overlap parameter so that $\mathcal{B}^{-1} \gg 1$.

Therefore we choose $\eta, \nu_{0}$ and $M$, under the constraints $\eta \ll 1$ and $\nu_{0} / M \ll 1$, such that $K_{\mathrm{D}}$ and $\Delta v_{\mathrm{D}}$ are sufficiently small while $\nu_{0} / M \lesssim \eta^{1 / 4}$ is small enough and $\nu_{0} \gg 1$ is large enough to balance the small value of $\eta$ in the estimate for $\mathcal{B}$ and so ensure a small value for this latter parameter.

\section{Hyperbolic case}

Following Doxas and Cary [15] we choose an initial distribution function $f_{0}$ such that the Landau growth rate is uniform in velocity. This choice is interesting for several reasons. It enables: (i) a simple validation of the numerical description of the linear regime; (ii) all waves (with various phase velocities) to enter more or less together into the nonlinear regime by choosing initial amplitudes provided by the saturation amplitude predicted by QL theory, downscaled by a (small) factor $\epsilon_{\zeta^{0}}$ independent from the velocity; (iii) continuity with the work [15]. Since

$$
\gamma_{\mathrm{L}}(0, v)=\frac{\pi}{2} \frac{\eta}{1+\eta} v^{2} \partial_{v} f_{0}(v)
$$

we obtain for all $v \in\left[u_{0}, u_{1}\right]$,

$$
f_{0}(v)=C_{1}+C_{2}\left(1-\frac{u_{0}}{v}\right)
$$


where

$$
C_{2}=\frac{2 \gamma_{\mathrm{L}}(1+\eta)}{\eta \pi u_{0}}, \quad \text { and } \quad C_{1}=\frac{1+C_{2}\left[u_{0} \ln \left(\frac{u_{1}}{u_{0}}\right)-u_{1}+u_{0}\right]}{u_{1}-u_{0}} .
$$

From momentum conservation we get

$$
\mathfrak{P}_{\mathrm{w}}^{\infty}-\mathfrak{P}_{\mathrm{w}}^{0}=\left(u_{1}-u_{0}\right)\left(\frac{u_{0}+u_{1}}{2}\left(C_{1}+C_{2}\right)-C_{2} u_{0}\right)-\frac{u_{0}+u_{1}}{2} .
$$

We initialize the wave amplitudes by setting

$$
\left|\zeta_{m}\right|=\epsilon_{\zeta^{0}} \sqrt{2 \psi_{\infty}\left(v_{m}\right) \frac{\Delta v_{m}}{k_{m}}}
$$

where, for all $v \in\left[u_{0}, u_{1}\right]$,

$\psi_{\infty}(v)=\int_{u_{0}}^{v}\left(f_{\infty}(\xi)-f_{0}(\xi)\right) \mathrm{d} \xi=\left(\frac{1}{u_{1}-u_{0}}-C_{1}-C_{2}\right)\left(v-u_{0}\right)+C_{2} u_{0} \ln \left(\frac{v}{u_{0}}\right)$

with $\epsilon_{\zeta^{0}}$ a sufficiently small positive real number such that an initial linear regime with an exponential growth of waves could exist. Equation (30) is obtained by integrating conservation law (24) with initial condition $\partial_{v} \psi(0, v)=0$ and boundary condition $\psi\left(t, u_{0}\right)=0$.

Therefore we choose an initial wave spectrum $\left\{\zeta_{m}\right\}=\left\{\left(\operatorname{Re} \zeta_{m}, \operatorname{Im} \zeta_{m}\right)\right\}=$ $\left\{\left(\left|\zeta_{m}\right| \cos \varphi_{m},\left|\zeta_{m}\right| \sin \varphi_{m}\right)\right\}$, such that wave amplitudes $\left|\zeta_{m}\right|$ are given by (29) and phases $\varphi_{m}$ are given by independent uniformly distributed real-valued random variables on the circle (i.e. with $1 /(2 \pi)$ for probability density). The physical parameters of the simulations are $\omega_{\mathrm{p}}=1, \nu_{0}=75, M=450, \gamma_{\mathrm{L}}(0)=10^{-3}, \eta=2.55 \times 10^{-3}, T_{\text {end }}=10^{4}$ $\left(\simeq 2 \max \left\{\left\langle\gamma_{\mathrm{L}}(0)\right\rangle^{-1}, T_{\text {dif }}\right\}\right), L_{x}=152 \pi, v_{\min }=0$ and $v_{\max }=8 / 7$. The numerical parameters of the simulations are $N_{x}=2112\left(n_{\delta x} \simeq 4\right), N_{v}=768\left(n_{\Delta v_{\varphi}} \simeq 1\right)$, $\Delta t=0.25\left(n_{\Phi} \simeq 4\right)$ and $\epsilon_{\zeta^{0}}=\exp (-8)$. Using the coarse estimates of section 4.2 we get the estimates for the plateau regime $K_{\mathrm{D}}^{-1} \simeq 30, s_{\mathrm{ov}} \simeq 50$ (or $\mathcal{B}^{-1} \simeq 36$ ) and $\Delta v_{\mathrm{D}} \simeq 3 . \times 10^{-2}$. Finally we have $u_{0}=0.145, u_{1}=1, k_{\min }=1, k_{\max } \simeq 6.91$, and $\Delta v_{\text {spec }}^{-1} \simeq 1.17$.

In contrast with [15] we do not impose a dense wave spectrum, with $s_{\text {ov }} \gg 1$, at initial time : $s_{\mathrm{ov}, m}(0) \simeq s_{\mathrm{ov}}\left(T_{\mathrm{end}}\right) \sqrt{\epsilon_{\zeta^{0}}} \simeq 50 \exp (-4) \simeq 1$. Besides, our initial wave amplitudes are not tuned to imply a $v$-independent $D_{\mathrm{QL}}$, but rather to fit the wave power spectrum in the plateau regime, as predicted by QL equations. The reason for this difference is that we do not focus specifically on regime INL to monitor $\gamma / \gamma_{\mathrm{L}}$ but rather on regime SNL reached in the plateau, where we want to compute $D / D_{\mathrm{QL}}$ for $\mu \rightarrow \infty$. However, we use the INL behaviour to benchmark our simulation with respect to $[15]$.

Table 1 sums up the parameter values used here (noted B.E.E.B.) and in [15] (noted D.C.) for the hyperbolic test case. The first three lines recall fixed parameters. The subsequent lines compare physical quantities $\gamma, \mu, s_{\mathrm{ov}}, K_{\mathrm{D}}$ at specific times in regime INL, at which the current wave growth rate $\gamma$ differs most from the Landau growth rate (two points $S_{1}$ and $S_{2}$ are considered for B.E.E.B. depending on the definition of average rates). 


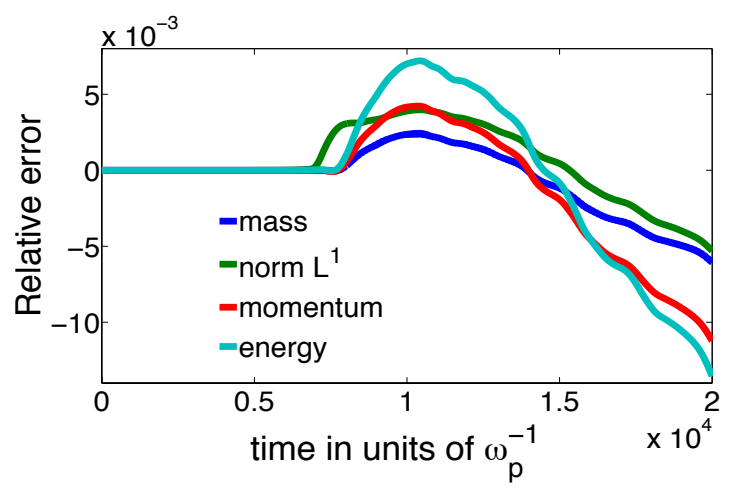

Figure 1. Relative error on conservation laws.

\subsection{Validation of the linear and nonlinear regimes}

This section is devoted to the code validation in the linear and nonlinear regimes. All figures refer to a single, typical realization of the dynamics.

We observe in figure 1 that mass is conserved with a relative error less than $0.25 \%$ up to time $1.6 \times 10^{4} \omega_{\mathrm{p}}^{-1}$. The conservation of the $L^{1}$-norm, which measures the importance of negative values in the distribution function, is very good since up to time $1.9 \times 10^{4} \omega_{\mathrm{p}}^{-1}$ it is preserved with a relative error less than $0.4 \%$. The conservation laws of momentum and energy are well respected, since momentum is conserved with a relative error less than $0.42 \%$ up to time $1.58 \times 10^{4} \omega_{\mathrm{p}}^{-1}$, while energy is conserved with a relative error less than $0.7 \%$ up to time $1.73 \times 10^{4} \omega_{\mathrm{p}}^{-1}$. Figures 2 show the shares of the total momentum and energy between wave, particle and coupling terms. Wave-particle coupling energy remains negligible, which supports the strong randomness of the microscopic dynamics in the nonlinear regimes. In figure 2-(a) we observe that momentum transfer from particles to waves is about $1 / 6$ of the total momentum.

Next we benchmark our simulation by checking the initial regime L. Since every wave grows exponentially in the linear stage with the same growth rate by construction, we can compare directly this theoretical growth rate with the growth rate of the waves total energy $\mathfrak{H}_{\mathrm{w}}$. Figure 3 shows that the theoretical growth rate of the waves coincides perfectly with the numerical one until time $t=2000 \omega_{\mathrm{p}}^{-1}$, which means that the system actually starts with an initial linear stage characterized by $\mu \ll 1$ with a duration of $2000 \omega_{\mathrm{p}}^{-1}=2 / \gamma_{\mathrm{L}}(0)$. This observation of the waves total energy, and of its growth rate $\gamma(t):=\left(2 \mathfrak{H}_{\mathrm{w}}\right)^{-1} \frac{\mathrm{d}}{\mathrm{d} t} \mathfrak{H}_{\mathrm{w}}$, will be confirmed below, by the analysis of the growth of all waves individually in figure 5.

From time $t=2000 \omega_{\mathrm{p}}^{-1}$ to $t=7000 \omega_{\mathrm{p}}^{-1}$ we observe an intermediate regime where the wave growth rates depart from Landau's linear approximation (see figures 3, 4-(a) and $5)$ : as noted by [15], mode coupling is present, and this intermediate stage corresponds to the transition regime INL, where parameter $\mu$ increases from a small value to a value of the order of unity or larger, and $s_{\text {ov }}$ increases from a moderate value to a large one. Because the particle velocity distribution function does not flatten at the same rate at 


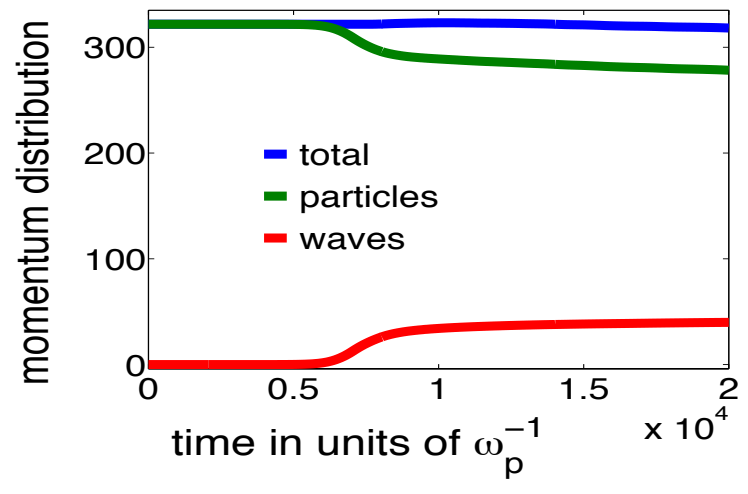

(a) Momentum sharing

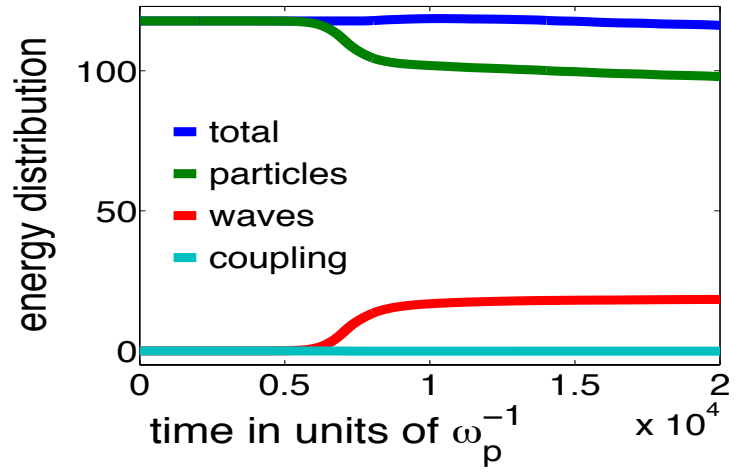

(b) Energy sharing

Figure 2. Distribution of momentum and energy.

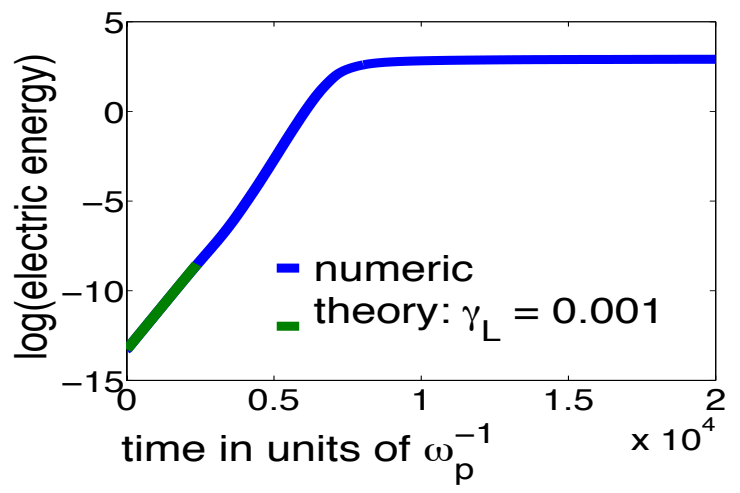

Figure 3. Logarithm of waves energy $\mathfrak{H}_{\mathrm{w}}$. 


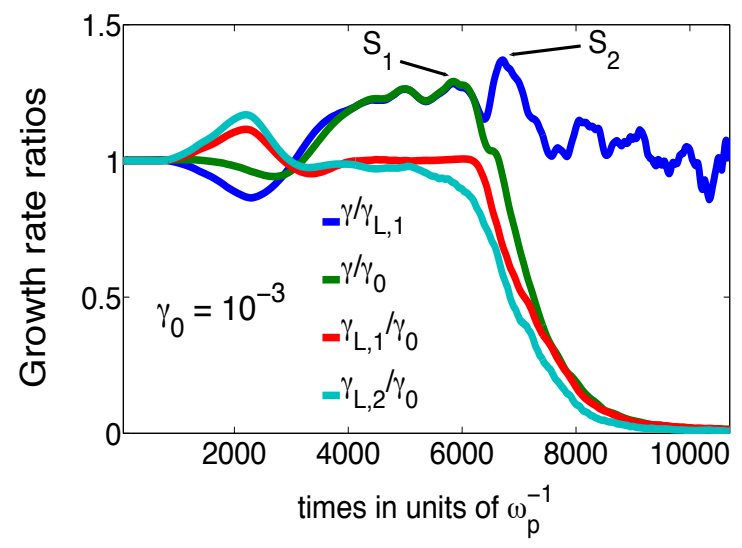

(a) Growth rate ratios

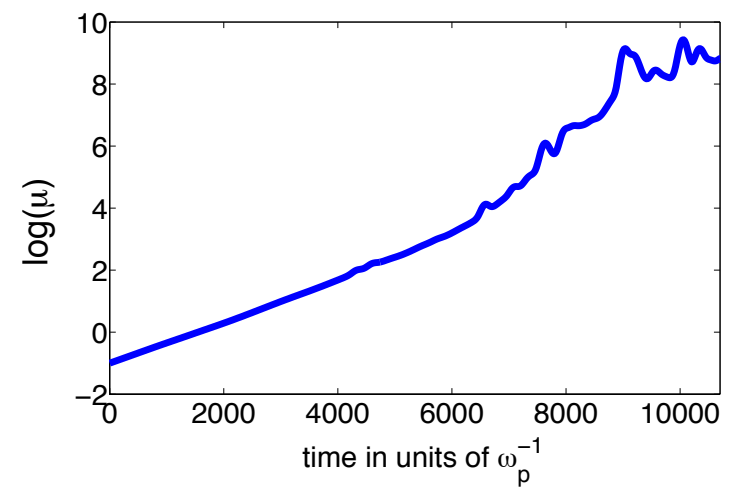

(b) $\log (\mu)$

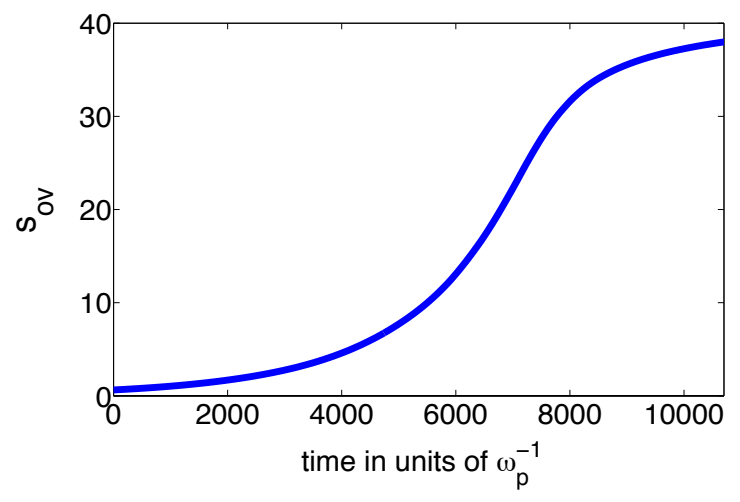

(c) $s_{\mathrm{ov}}$

Figure 4. Time evolution of $\gamma / \gamma_{\mathrm{L}}, \gamma / \gamma_{\mathrm{L}}(0), \gamma_{\mathrm{L}} / \gamma_{\mathrm{L}}(0), \log (\mu)$ and $s_{\mathrm{ov}}$. Here the energy growth rate is $\gamma(t)=\left(2 E_{\mathrm{w}}(t)\right)^{-1} \mathrm{~d} E_{\mathrm{w}}(t) / \mathrm{d} t$, where $E_{\mathrm{w}}(t)=$ $\sum_{m=10}^{M}\left|\zeta\left(t, v_{m}\right)\right|^{2}$ (with $v_{10} \simeq 0.9$ ). Two average Landau growth rates are plotted, viz. $\quad \gamma_{\mathrm{L}, 1}(t)=\left(\sum_{m=10}^{M} \Delta v_{m}\right)^{-1} \sum_{m=10}^{M} \gamma_{\mathrm{L}}\left(t, v_{m}\right) \Delta v_{m}$ and $\gamma_{\mathrm{L}, 2}(t)=$ $\left(\sum_{m=10}^{M}\left|\zeta\left(t, v_{m}\right)\right|^{2}\right)^{-1} \sum_{m=10}^{M} \gamma_{\mathrm{L}}\left(t, v_{m}\right)\left|\zeta\left(t, v_{m}\right)\right|^{2}$. 


\begin{tabular}{|c|c|c|c|}
\hline Test case & B.E.E.B. $S_{1}$ & B.E.E.B. $S_{2}$ & D.C. \\
\hline$M$ & 450 & 450 & 584 \\
\hline$\gamma_{0}$ & $10^{-3}$ & $10^{-3}$ & $6.25 \times 10^{-5}$ \\
\hline$\eta$ & $2.55 \times 10^{-3}$ & $2.55 \times 10^{-3}$ & $4.1 \times 10^{-6}$ \\
\hline $\max \gamma / \gamma_{\mathrm{L}, 1}$ & 1.29 & 1.36 & 1.32 \\
\hline$\mu$ & 22 & 56 & 39 \\
\hline$s_{\mathrm{OV}}$ & 12 & 20 & 14 \\
\hline$K_{\mathrm{D}}^{-1}$ & 1950 & 660 & 94 \\
\hline
\end{tabular}

Table 1. Comparaison of physical parameters at time where $\gamma / \gamma_{\mathrm{L}}$ is maximum between our test case (B.E.E.B.) and test case of [15] (D.C.).

all velocities, the Landau growth rate depends on $v$, and we monitor two average rates, viz. a crude average $\gamma_{\mathrm{L}, 1}(t)=\left(\sum_{m=10}^{M} \Delta v_{m}\right)^{-1} \sum_{m=10}^{M} \gamma_{\mathrm{L}}\left(t, v_{m}\right) \Delta v_{m}$ and an intensitybased average $\gamma_{\mathrm{L}, 2}(t)=\left(\sum_{m=10}^{M}\left|\zeta\left(t, v_{m}\right)\right|^{2}\right)^{-1} \sum_{m=10}^{M} \gamma_{\mathrm{L}}\left(t, v_{m}\right)\left|\zeta\left(t, v_{m}\right)\right|^{2}$, discarding the contribution of waves too close to the "plateau" edge (which we saw in section 2.2 to possibly behave differently from the bulk of the wave spectrum, and which are affected here by the steepening of $f$, see figure 6 ). Both average rates behave similarly, and we shall not dwell on their differences as our simulations were tailored to assess a possible renormalization of the diffusion coefficient in the plateau regime. During the beginning of this regime the perturbative calculation of [38] makes likely the occurrence of an enhanced growth rate. What is striking is the growth rate enhancement by a factor greater than 1.2 over the interval $t \in\left[4000 \omega_{\mathrm{p}}^{-1}, 7000 \omega_{\mathrm{p}}^{-1}\right]$, with a maximum factor 1.36 at time $t=6731 \omega_{\mathrm{p}}^{-1}\left(\mu \simeq 56, s_{\mathrm{ov}} \simeq 20\right)$, confirming the saturation value emerging in [15] (see table 1). This enhancement contradicts the analytical argument for the validity of quasilinear theory in [51], since our numerical calculation involves the averages over a Dupree width. Actually, assessing [51] does not even require such an average, as the wave intensities are a smooth function of velocity for this run in this time range. This enhanced growth rate by 1.36 may be related to the enhanced particle transport (by up to $2.3 \simeq 1.36^{3}$, see the scaling $\tau_{\mathrm{D}} \sim D^{-1 / 3}$ ) in a given wave field (9) with smooth intensity and random phases $[13,20]$.

We next observe a nonlinear saturation stage from time $t=7000 \omega_{\mathrm{p}}^{-1}$ to time $t=2 \times 10^{4} \omega_{\mathrm{p}}^{-1}$, where the plateau is set up by time $t=8400 \omega_{\mathrm{p}}^{-1}$, in figures 3,5 , and 6 .

In figures 4 , we observe that for relatively large values of $\mu$, typically $\mu \simeq 56$, the ratio $\gamma / \gamma_{\mathrm{L}}$ is around 1.36 (see table 1 ). In $[42,43]$ the authors obtain an analytical formula (equation (38) in [43]) for such a renormalization factor $\gamma_{\text {ren }}$ which scales like $\gamma_{\text {ren }}=1+A \gamma_{\mathrm{L}} / \omega_{\mathrm{p}}$ in the regime where $\mu \gg 1$, with a factor $A$ of the order of unity. Of course this asymptotic expression of $\gamma_{\text {ren }}$ holds in the plateau regime since in this

I The small decrease of $\gamma$ for $2000<\omega_{\mathrm{p}} t<3000$ (with $\gamma / \gamma_{0} \sim 0.95, \mu \simeq 2.1$ and $s_{\text {ov }} \simeq 2.2$ ) remains tolerable in our benchmarking, as [15] also obtains $\gamma / \gamma_{0} \sim 0.98$ with $\mu \simeq 2.2$ and $s_{\text {ov }} \simeq 14$. 
case $\gamma_{\mathrm{L}} \simeq 0$. Nevertheless equation (38) in [43] is not compatible with the result of figures 4 since in the regime where $\mu \gg 1$, there exists an intermediate stage (regime INL) where we observe an enhancement of the growth rate with a factor around 1.36. This enhancement is significantly smaller than the turbulent trapping estimate which may be as large as 2 . Yet a more detailed inspection in figure 5 of the growth rate $\gamma(t, v)=\frac{1}{2} \psi^{-1} \mathrm{~d} \psi / \mathrm{d} t=|\zeta|^{-1} \mathrm{~d}|\zeta| / \mathrm{d} t$ shows that the ratio $1.2 \ldots 1.36$ merely reflects an average behaviour, and that actually the wave intensities burst quite violently but also quite independently from each other during this time range.

Finally, figure 4-(a) shows that after a period where the enhancement factor increases to its maximum value 1.36 , there exists a second stage where this factor decreases, which means that the enhancement process breaks down after a certain time in the INL regime. This result contradicts the hypothesis of [43], according to which the enhanced growth rate or the enhanced diffusivity could be the result of an artificial effect caused by imposing periodic boundary conditions in numerical simulations. Therefore periodicity does not seem here to impact artificially the enhancement of nonlinear effects (furthermore, if it had such an impact, the argument would also apply in the plateau regime - which it clearly does not, see below).

Note that when the plateau is settled, say for times $t>8000 \omega_{\mathrm{p}}^{-1}$, both $\gamma$ and $\gamma_{\mathrm{L}}$ are small, so that their ratio becomes numerically less meaningful.

\subsection{Transition from stochastic to chaotic diffusion}

After discussing the global aspects of the Vlasov-wave system evolution, we turn to more detailed behaviour, resolved in velocity $v$.

From figures 6-(b) and 6-(c) we observe that the space-averaged distribution function evolves from the hyperbolic shape to the plateau one between time $t \simeq 4800 \omega_{\mathrm{p}}^{-1}$ and time $t \simeq 8400 \omega_{\mathrm{p}}^{-1}$; figure 6-(c) shows that the plateau appears near the center of the velocity distribution and erodes the higher values of $f$ causing the increase in the wave growth rate for faster phase velocities. Simultaneously the growth rate of every wave falls from a strictly positive value to a vanishing one (see figure 5). This means that the plateau regime is reached by time $t \simeq 8400 \omega_{\mathrm{p}}^{-1}$, as confirmed by figure 7 .

In figures 8 and 10 we analyse the velocity profiles of wave spectrum intensity (30) and quasilinear diffusion coefficient. The smooth curves are the theoretical predictions (4) obtained by integrating the conservation law (24) with initial condition $\partial_{v} \psi(0, v)=0$ and boundary condition $\psi\left(t, u_{0}\right)=0$. We compare this prediction with the result of a single realization of initial data (figures 9 and 11), and with the average spectrum for a statistical ensemble comprising $R=210$ realizations of the same Vlasov-wave test case where random initial phases are drawn independently from a uniform distribution on the circle (figures 8 and 10). In the sequel the bracket notation $\langle\cdot\rangle_{R}$ means that averaging over a statistical ensemble of $R$ realizations has been performed. In figures 8(b) and 10-(b) the "avg" index means that we carried out a floating average of five wave 


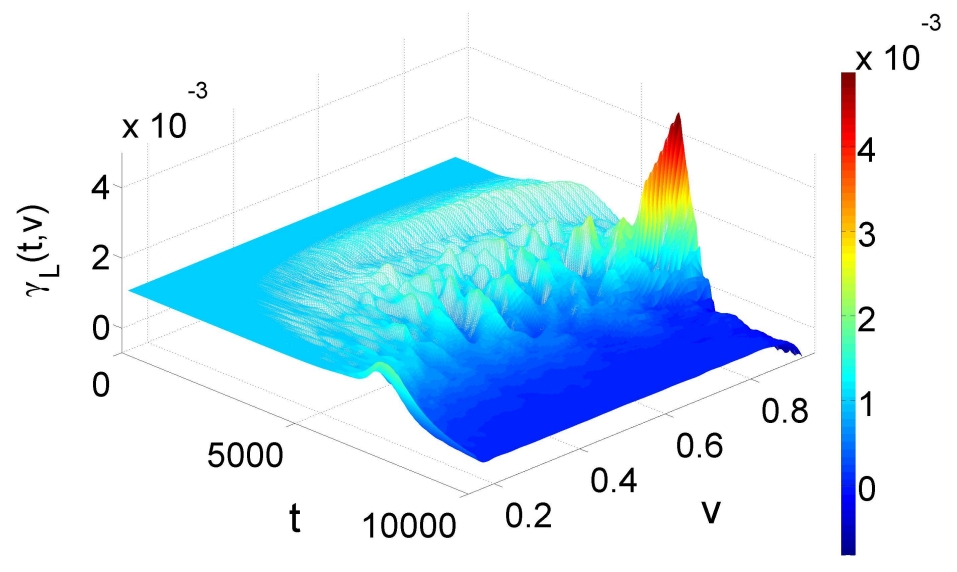

(a) $\gamma_{\mathrm{L}}(t, v)$

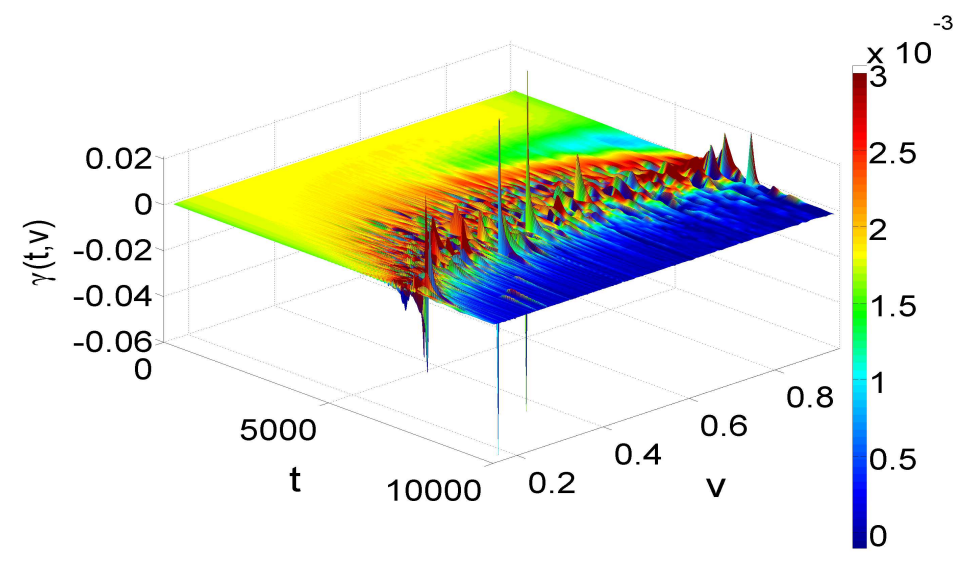

(b) $\gamma(t, v)$

Figure 5. Time evolution of growth rates. (a) $\gamma_{\mathrm{L}}$ given by (3) from the current $f(t, v)$; (b) individual wave growth rate measured from wave intensity evolution.

half-widths, viz. we set (with $L_{\text {avg }}=5$ )

$$
D_{\mathrm{QL}, \mathrm{avg}}\left(v_{m}\right)=\sum_{\ell=-L_{\mathrm{avg}}}^{L_{\mathrm{avg}}} \frac{D_{\mathrm{QL}}\left(v_{m}\right) \Delta v_{\varphi, m+\ell}}{v_{\varphi, m+L_{\mathrm{avg}}+1}-v_{\varphi, m-L_{\mathrm{avg}}}} .
$$

From figures 8 and 10 in the plateau regime, we observe quite a good agreement between the QL prediction of the wave spectrum (resp. diffusion coefficient) and the one obtained by self-consistent simulations of the Vlasov-wave model.

In order to verify that we are in regime SNL where chaotic diffusion applies, we need to look at the value of the Kubo parameter $K_{\mathrm{D}}$, of the Chirikov resonance overlap parameter $s_{\text {ov }}\left(\right.$ or $\left.\mathcal{B}^{-1}\right)$ and of parameter $\mu$. From figures 12 we notice that at the middle of the phase velocity range of the wave spectrum, i.e. for $v_{\mathrm{c}}=\left(u_{0}+u_{1}\right) / 2 \simeq 0.57$, and at time $T=10^{4} \omega_{\mathrm{p}}^{-1}$ in the plateau regime, we have $\left\langle s_{\mathrm{ov}}\right\rangle_{R}\left(T, v_{\mathrm{c}}\right) \simeq 22,\left\langle K_{\mathrm{D}}^{-1}\right\rangle_{R}\left(T, v_{\mathrm{c}}\right) \simeq 21$, and $\langle\mu\rangle_{R}\left(T, v_{\mathrm{c}}\right) \simeq 4900$. These values confirm that the system is in regime SNL. 


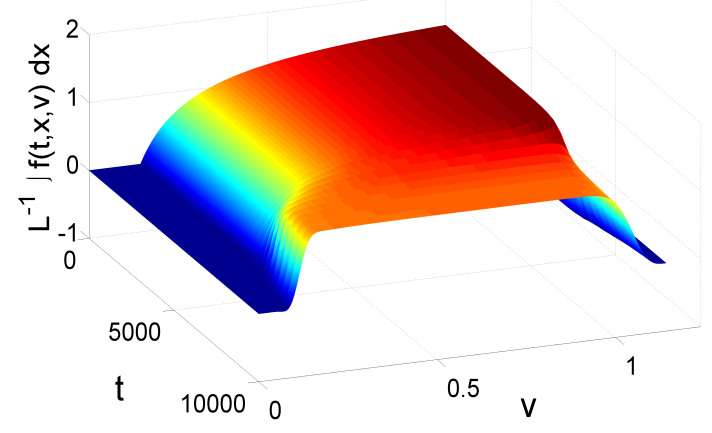

(a) $\frac{1}{L} \int f(t, x, v) \mathrm{d} x$

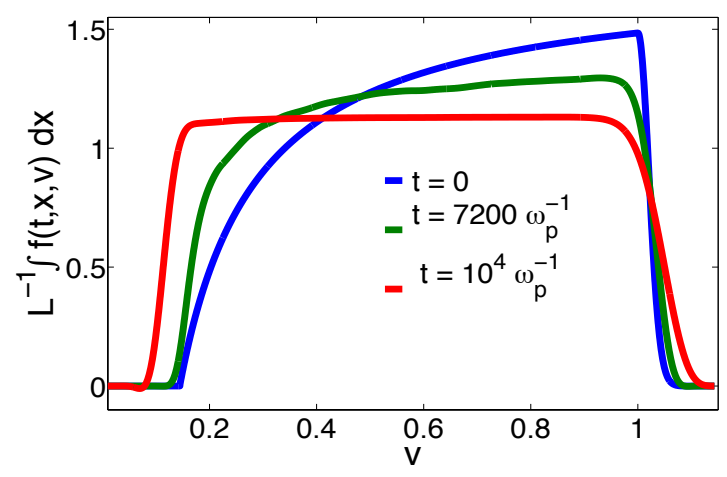

(b) $\frac{1}{L} \int f(t, x, v) \mathrm{d} x$

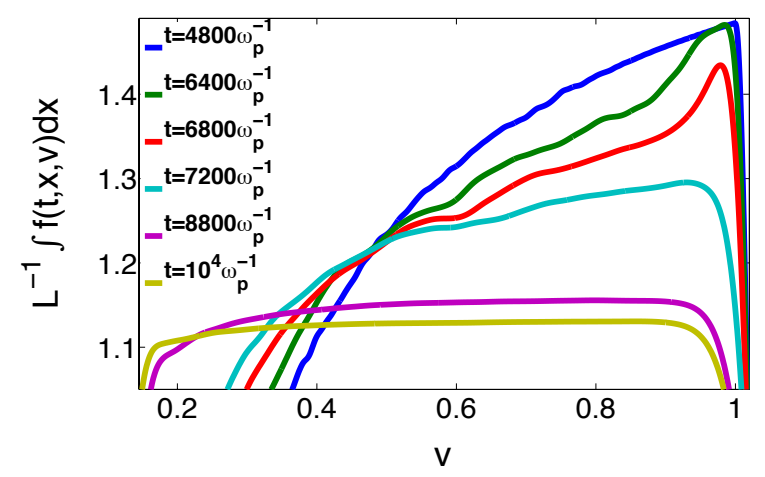

(c) $\frac{1}{L} \int f(t, x, v) \mathrm{d} x$

Figure 6. $x$-averaged velocity distribution function.

As expected from section 2.3, once the plateau regime has been reached (see figures 5 and 6) the time dependence of the amplitude spectrum is weak (see figure 7). Therefore we may neglect this dependence and consider that the self-consistent dynamics can be approximated by the non-self-consistent one, generated by (9). Indeed when the distribution function has reached the plateau shape both in velocity and in position, the Fourier coefficients in (16) are almost zero (because $f$ is constant), bringing time variation of the waves to rest: the Vlasov equation lands on the non-self-consistent 


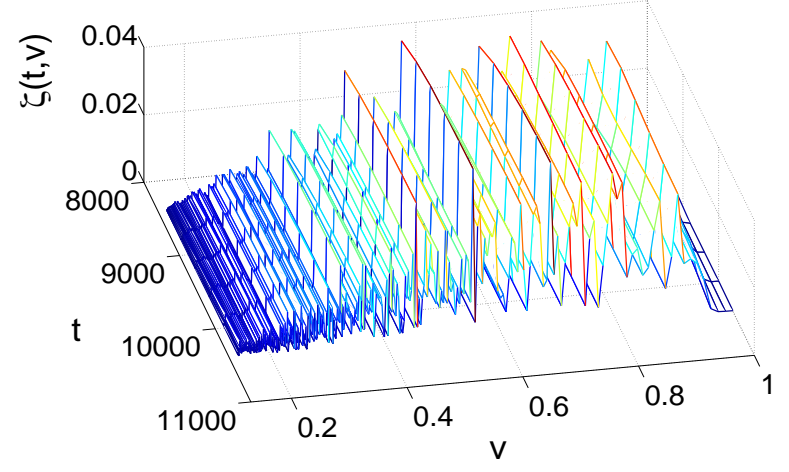

Figure 7. Time evolution of wave intensity spectrum $|\zeta(t, v)|$, from time $t=8400 \omega_{\mathrm{p}}^{-1}$ to time $t=10400 \omega_{\mathrm{p}}^{-1}$.

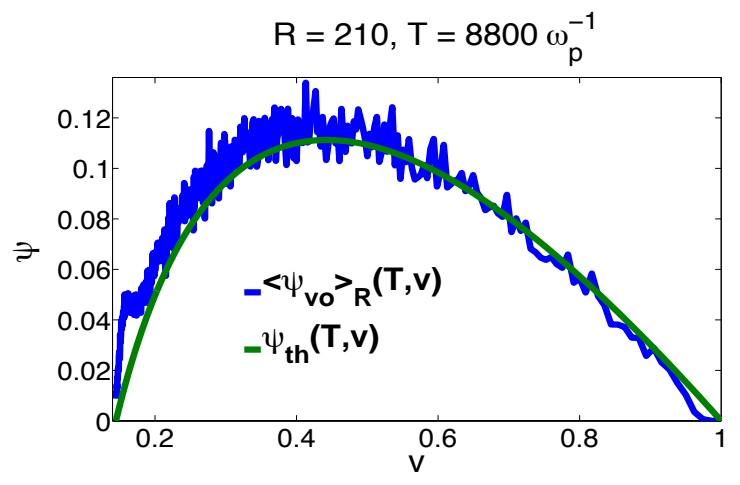

(a) $\langle\psi\rangle_{R}(T, v)$ vs. $\psi_{\text {th }}(T, v)$

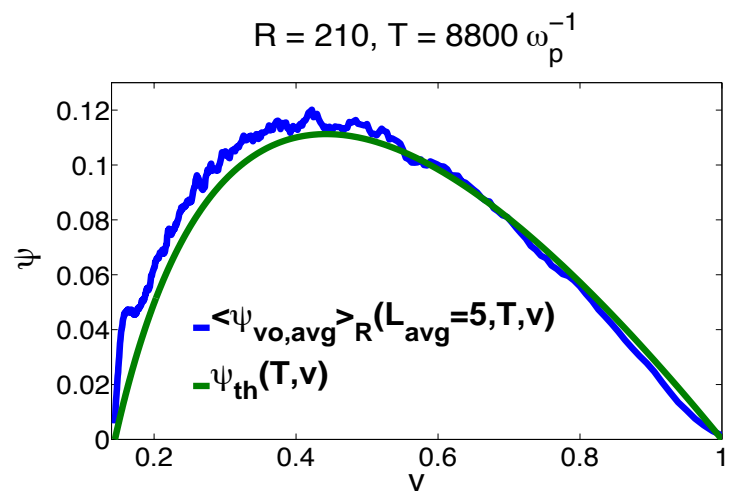

(b) $\left\langle\psi_{\text {avg }}\right\rangle_{R}(T, v)$ vs. $\psi_{\text {th }}(T, v)$

Figure 8. Ensemble averaged wave intensity spectrum. 


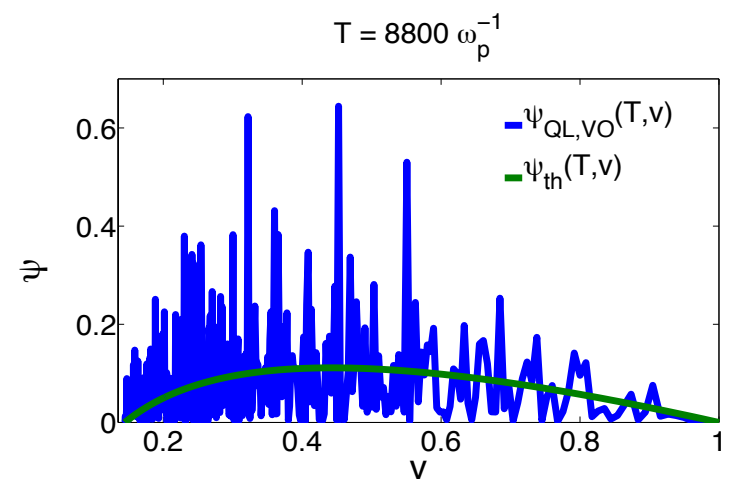

(a) $\psi(T, v)$ vs. $\psi_{\text {th }}(T, v)$

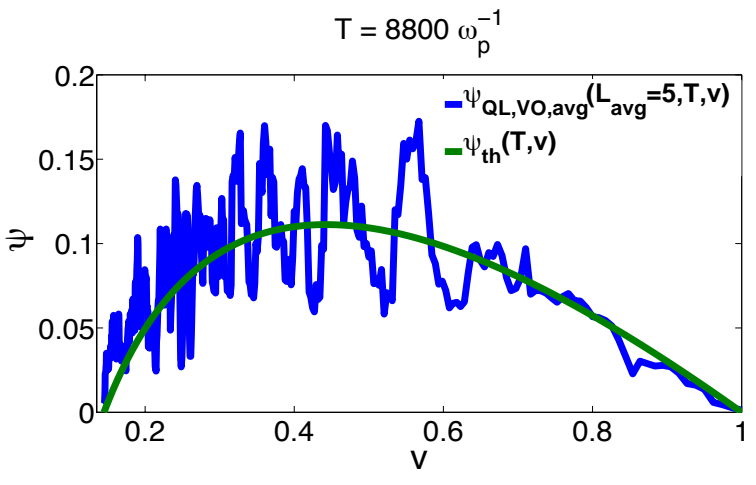

(b) $\psi_{\text {avg }}(T, v)$ vs. $\psi_{\text {th }}(T, v)$

Figure 9. Typical wave intensity spectrum for a single realization.

configuration. The works on Hamiltonian chaotic dynamics (9), recalled in the introduction and more precisely in Appendix A, show that QL estimates hold if the wave spectrum satisfies two sufficient assumptions.

The first one requires the wave amplitude spectrum not to have holes larger than the order of a width $\Delta v_{\mathrm{D}}$. From figures 8 (resp. figures 10), we observe that the typical amplitudes of the waves (resp. diffusion coefficient) are smooth enough with respect to their velocity (or wavenumber).

Even if nearby waves seem to have strongly inhomogeneous amplitude values (resp. diffusion coefficients) with respect to velocity in figure 9-(a) (resp. figure 11-(a)), once averaged over a velocity width corresponding to a floating average (31) with $L_{\text {avg }}=5$, wave spectrum amplitudes (resp. diffusion coefficients) appear smooth with respect to velocity in figure 9-(b) (resp. figure 11-(b)). Our floating average is very conservative: it should really be performed with the $v$-dependent range $L_{\text {avg, } \mathrm{D}}(t, v)=\Delta v_{\mathrm{D}} / \Delta v_{\varphi}=$ $\tau_{\text {discr }} / \tau_{\mathrm{D}}=\mathcal{B}^{-1}$, which is of the order of 22 near the middle of the wave spectrum (see figures 12). Therefore the first requirement, namely the absence of holes larger than the order of a width $\Delta v_{\mathrm{D}}$, is satisfied.

The second requirement on the wave spectrum is randomness of the final phase. Figure 13 shows the phases at $t=10^{4} \omega_{\mathrm{p}}^{-1}$ for a single, typical run, with random initial 


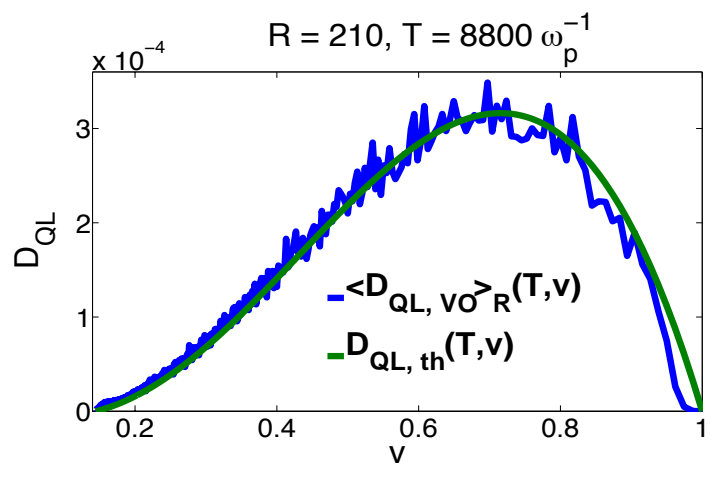

(a) $\left\langle D_{\mathrm{QL}}\right\rangle_{R}(T, v)$ vs. $D_{\mathrm{QL}, \mathrm{th}}(T, v)$

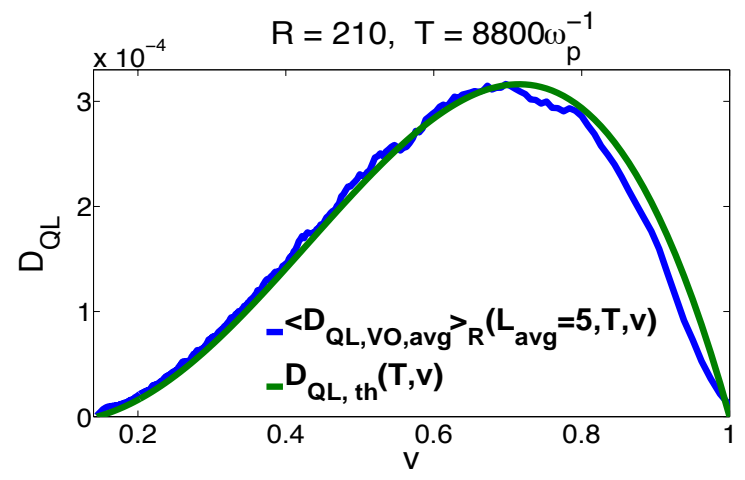

(b) $\left\langle D_{\mathrm{QL}, \text { avg }}\right\rangle_{R}(T, v)$ vs. $D_{\mathrm{QL}, \mathrm{th}}(T, v)$

Figure 10. Ensemble averaged quasilinear diffusion coefficient.

phases uniformly distributed on the circle: the wave evolution does not generate strong phase correlations between waves, and waves with neighbouring velocities have quite independent phases. In figures 14 we observe that the final phases appear to be drawn independently from a uniform distribution on the circle as was the case at initial time. Therefore the numerical wave spectrum fulfils the conditions leading to QL estimates.

Finally, we assess the validity of the QL diffusive model for particle motion in the plateau regime, by observing the spreading of $N=20$ test particles for each of the $R=210$ realizations of the wave complex envelopes $\zeta_{m}\left(t_{0}\right)$ at $t_{0}=8800 \omega_{\mathrm{p}}^{-1}$. These particle motions are obtained by direct integration of their equations of motion (11)-(12) with fixed $\zeta_{m}$, using a reversible symplectic second order code. The resulting statistics are displayed in terms of the even moments of the "test" particles released at $t_{0}$ with velocity $v_{\mathrm{c}}=\left(u_{0}+u_{1}\right) / 2$ at independent (uniformly distributed) random positions in the wave spectrum; an estimate for the numerical accuracy of the test particle motion is provided by the standard deviations of the moments.

In figures 15 these moments are compared with those of the solution to the Fokker-Planck equation (1) for velocity diffusion, using the velocity-dependent diffusion constant $D_{\mathrm{QL}}$ displayed in figure 10-(a), with initial data a Dirac distribution at velocity $v_{\mathrm{c}}$. To allow for a direct observation of the velocity distribution departure from 


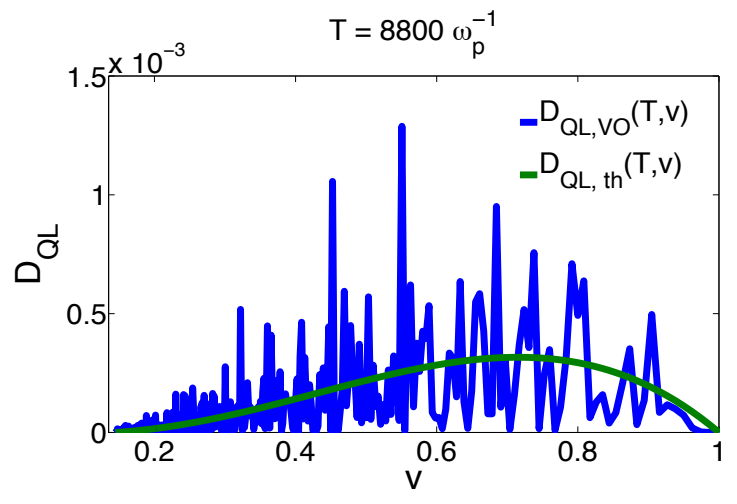

(a) $D_{\mathrm{QL}}(T, v)$ vs. $D_{\mathrm{QL}, \mathrm{th}}(T, v)$

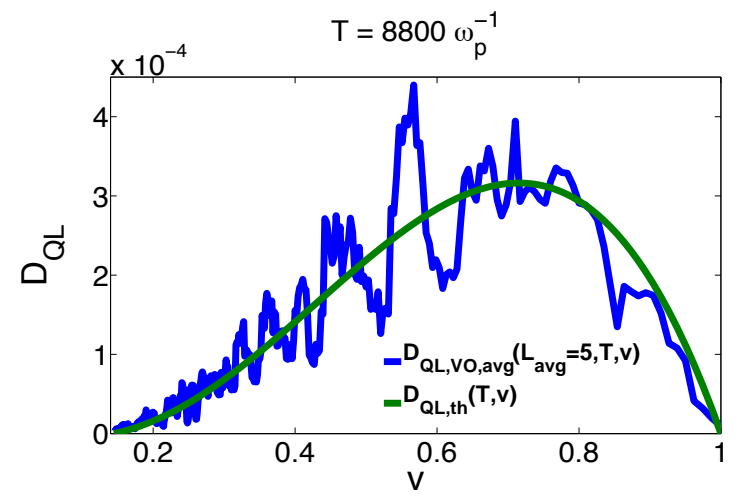

(b) $D_{\mathrm{QL}, \text { avg }}(T, v)$ vs. $D_{\mathrm{QL}, \mathrm{th}}(T, v)$

Figure 11. Typical quasilinear diffusion coefficient for a single realization.

gaussianity, we plot the moment roots $\left\langle|\Delta v(t)|^{2 k} /(1 \cdot 3 \ldots(2 k-1))\right\rangle^{1 / k}$, which would coincide with the second moment if the distribution were gaussian [20]. In these figures, time is normalized with the discretization time $\tau_{\text {discr }}$ associated with velocity $v_{\mathrm{c}}$, and $\Delta v(t)$ is normalized so that, for pure diffusion over an infinite domain with constant $D_{\mathrm{QL}}$, all plots would condense to a single straight line with unit slope.

We may consider the agreement with quasilinear predictions as satisfactory, given that there is no adjustable parameter in the modelling and that the spreading width (over which the diffusion approximation is expected to hold possibly) is not very small in comparison to the total wave spectrum width $\Delta v_{\text {spec }}$ : the drawback of the large ratio $\Delta v_{\mathrm{D}} / \Delta v_{\varphi}=\tau_{\text {discr }} / \tau_{\mathrm{D}}=\mathcal{B}^{-1}$ (for the given ratio $\Delta v_{\text {spec }} / \Delta v_{\varphi}$ of the wave spectrum) is that diffusing particles may reach the plateau boundary soon, ceasing then to contribute to the growth of the moments, and undergo there (influenced by KAM-like boundaries) a motion which may depart from the diffusive QL approximation further than near the centre of the plateau. There is thus no compelling indication for rejecting the simple quasilinear model (1), with coefficient $D_{\mathrm{QL}}(v)$, when describing particle transport in the plateau regime of the full Vlasov-waves system. 


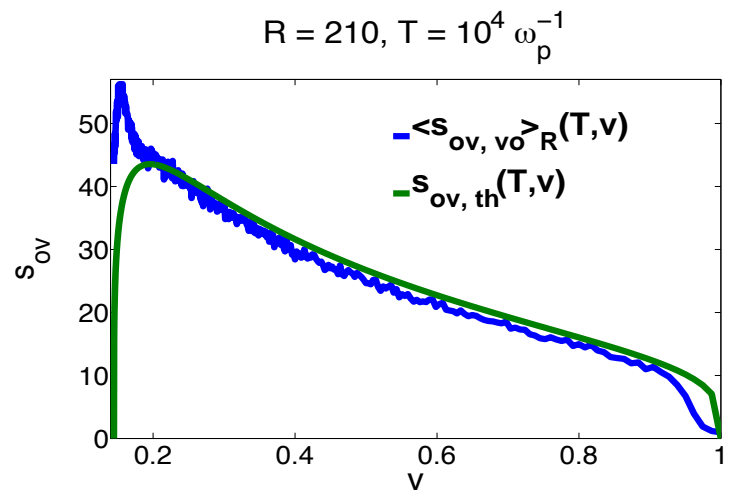

(a) $\left\langle s_{\mathrm{ov}}\right\rangle_{R}(T, v)$ vs. $s_{\mathrm{ov}, \mathrm{th}}(T, v)$

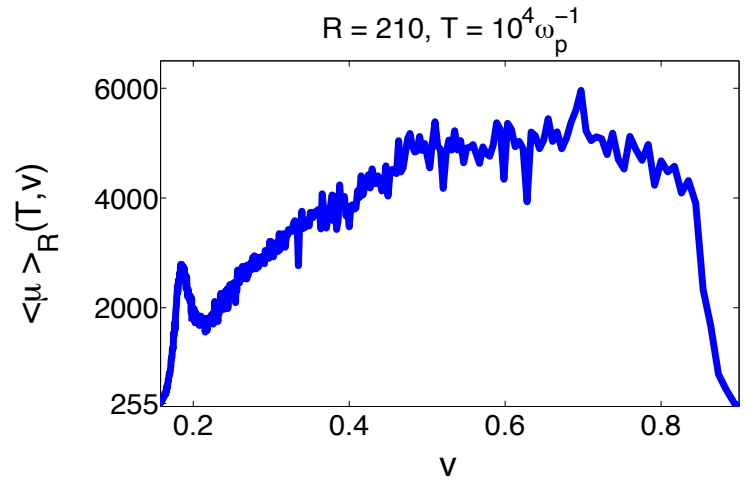

(b) $\langle\mu\rangle_{R}(T, v)$

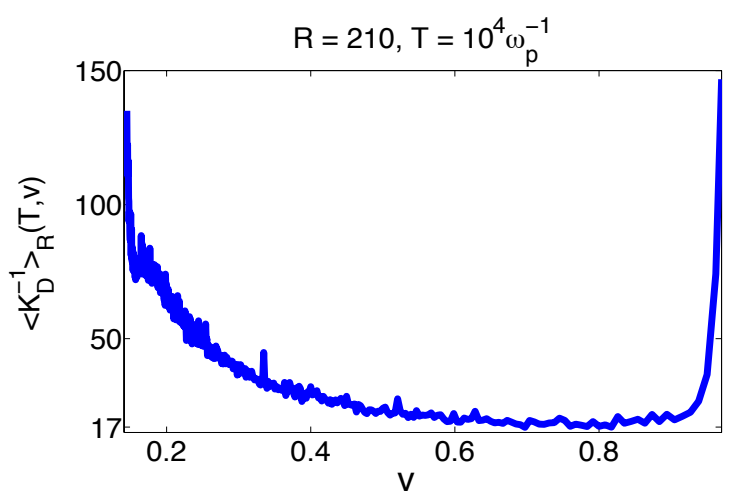

(c) $\left\langle K_{\mathrm{D}}^{-1}\right\rangle_{R}(T, v)$

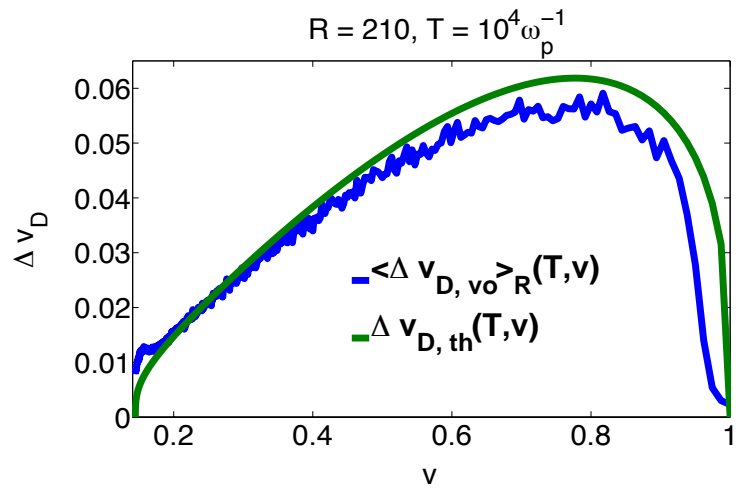

(d) $\left\langle\Delta v_{\mathrm{D}}\right\rangle_{R}(T, v)$ vs. $\Delta v_{\mathrm{D}, \text { th }}(T, v)$

Figure 12. Physical parameters. 


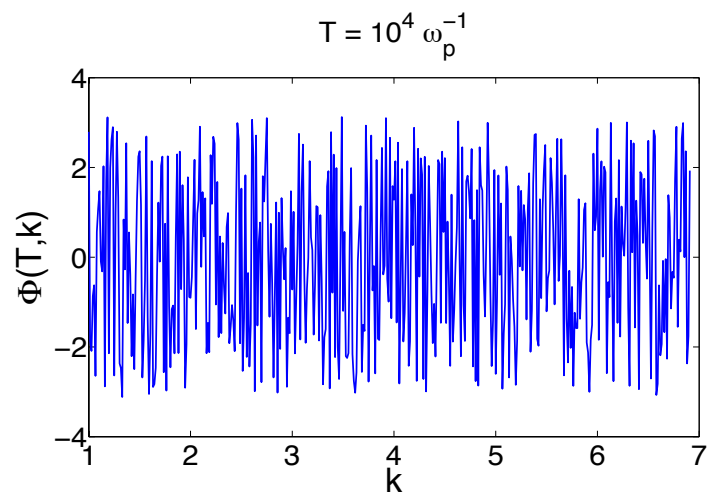

Figure 13. Typical final phase of the wave spectrum for a single realization.

\section{Triangular case}

In this section we consider a triangular initial distribution function $f_{0}$, which has the advantage of yielding more easily a large number of spreading widths $\Delta v_{\mathrm{D}}$ over the plateau width $\Delta v_{\text {spec }}$ at the expense of a larger computation time though. We performed fewer runs $(R=13)$ and discuss them here to stress the genericity of the behaviours observed above in the saturation regime.

The triangle vertices are $\left(u_{0}, 0\right),\left(u_{1}, 2 / \Delta v_{\text {spec }}\right)$ and $\left(u_{1}, 0\right)$, defining the initial distribution $f_{0}(v)=2\left(v-u_{0}\right) / \Delta v_{\text {spec }}^{2}$ for all $v \in\left[u_{0}, u_{1}\right]$ and 0 elsewhere. Momentum conservation yields

$$
\mathfrak{P}_{\mathrm{w}}^{\infty}-\mathfrak{P}_{\mathrm{w}}^{0}=\frac{\Delta v_{\mathrm{spec}}}{6}
$$

We choose an initial wave spectrum $\left(\left\{\left|\zeta_{m}\right|\right\},\left\{\varphi_{m}\right\}\right)$ such that the wave amplitude $\left|\zeta_{m}\right|$ is given by (29) with

$$
\psi_{\infty}(v)=\int_{u_{0}}^{v}\left(f_{\infty}(\xi)-f_{0}(\xi)\right) \mathrm{d} \xi=\frac{\left(u_{1}-v\right)\left(v-u_{0}\right)}{\left(u_{1}-u_{0}\right)^{2}}
$$

and phases $\varphi_{m}$ are given by independent uniformly distributed random variables on the real circle. The physical parameters of the simulations are $\omega_{\mathrm{p}}=1, \nu_{0}=200, M=1200$, $\eta=10^{-5}, T_{\text {end }}=9 \times 10^{5}\left(\simeq 2 \max \left\{\left\langle\gamma_{\mathrm{L}}(0)\right\rangle^{-1}, T_{\text {dif }}\right\}\right), L_{x}=402 \pi, v_{\min }=0.09$ and $v_{\max }=1.1$. The numerical parameters of the simulations are $N_{x}=5632\left(n_{\delta x} \simeq 4\right)$, $N_{v}=1408\left(n_{\Delta v_{\varphi}} \simeq 1\right), \Delta t=0.25\left(n_{\Phi} \simeq 4\right)$ and $\epsilon_{\zeta^{0}}=\exp (-4.5)$. The coarse estimates of section 4.2 yield for the plateau $K_{D}^{-1} \simeq 144, s_{\text {ov }} \simeq 32\left(\right.$ or $\left.\mathcal{B}^{-1} \simeq 20\right)$ and $\Delta v_{\mathrm{D}} \simeq 6.1 \times 10^{-3}$. Finally we have $u_{0}=0.144, u_{1}=1, k_{\min }=1, k_{\max }=6.97$, and $1 /\left(u_{1}-u_{0}\right)=1.17$.

\subsection{Validation of the linear and nonlinear regimes}

We first validate the code in the linear and nonlinear regimes. Figures 16 show that the mass and the $L^{1}$-norm (undistinguishable in the figure) are conserved with a relative error less than $0.05 \%$ up to time $9 \times 10^{5} \omega_{\mathrm{p}}^{-1}$. Total momentum and energy are well 


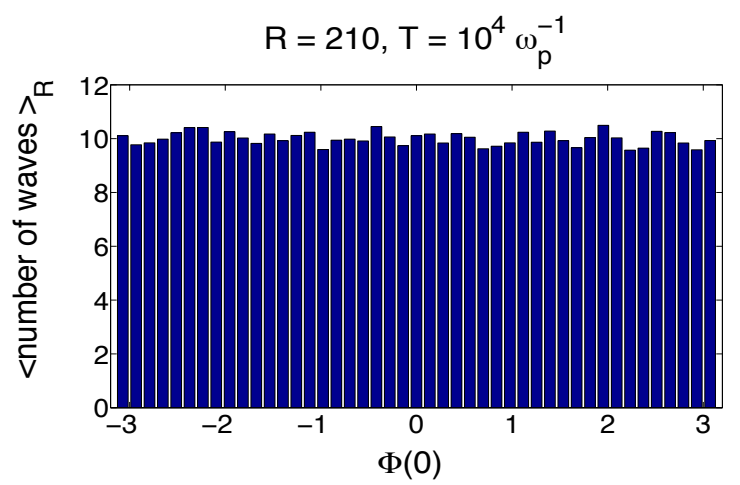

(a) Initial phase

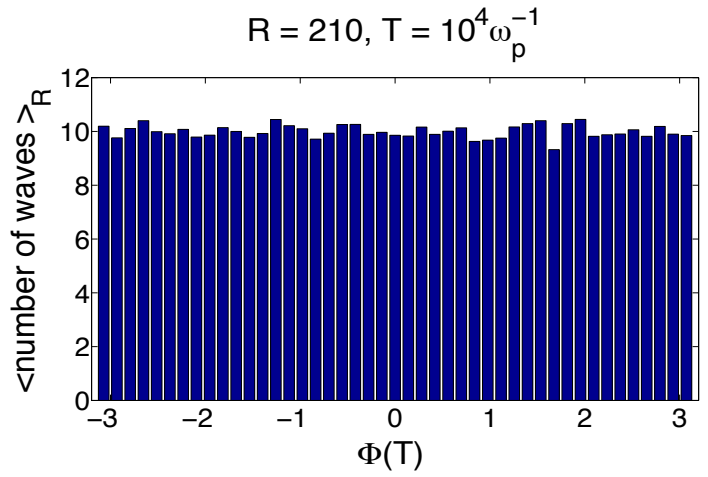

(b) Final phase

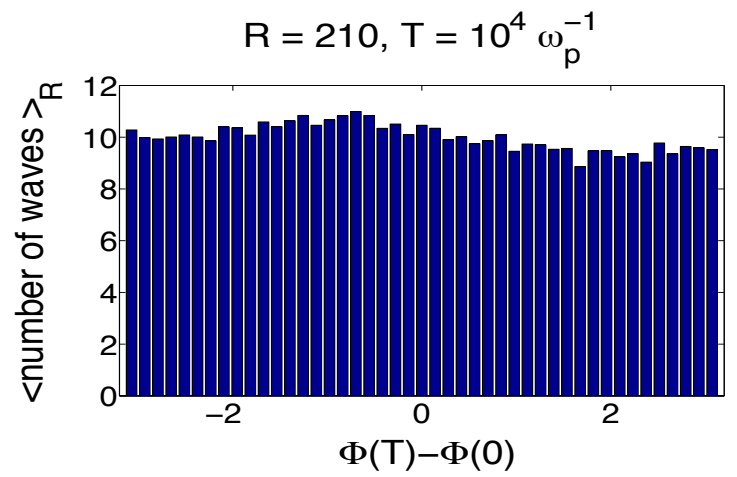

(c) Difference of initial and final phase

Figure 14. Distribution of the phase of the wave spectrum.

preserved, respectively with a relative error less than $0.08 \%$ and $0.06 \%$ up to the final time $9 \times 10^{5} \omega_{\mathrm{p}}^{-1}$. Figures 17 show the distribution of the total momentum and energy between wave, particle and coupling terms. In figure 17-(a) we observe that momentum transfer from particles to waves is about $1 / 5$ of the total momentum (which is of the order of QL estimate (32)) while figure 17-(b) indicates an energy transfer of about the quarter of the total energy. Wave-particle coupling energy remains negligible, which supports again the strong randomness picture of the microscopic dynamics in the 


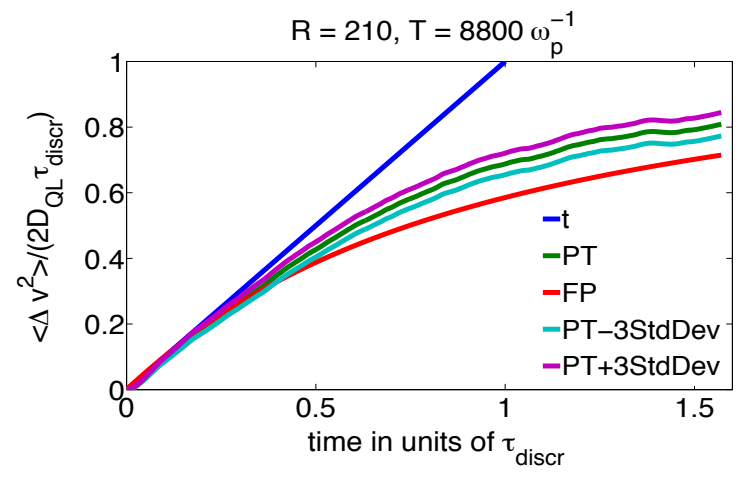

(a) $\mathfrak{M}_{2}$

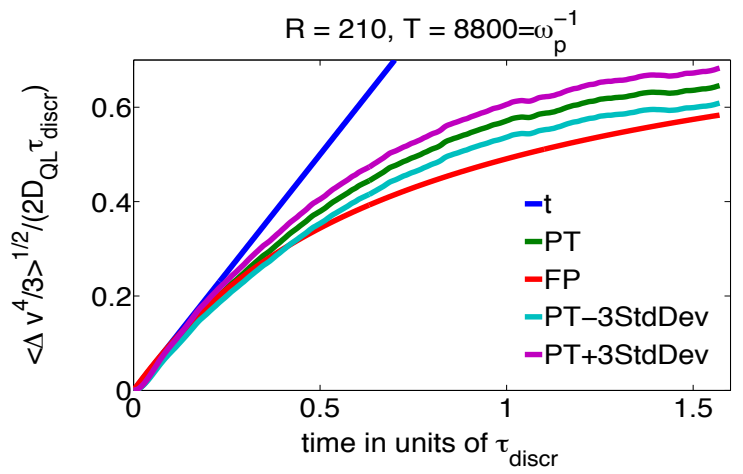

(b) $\mathfrak{M}_{4}$

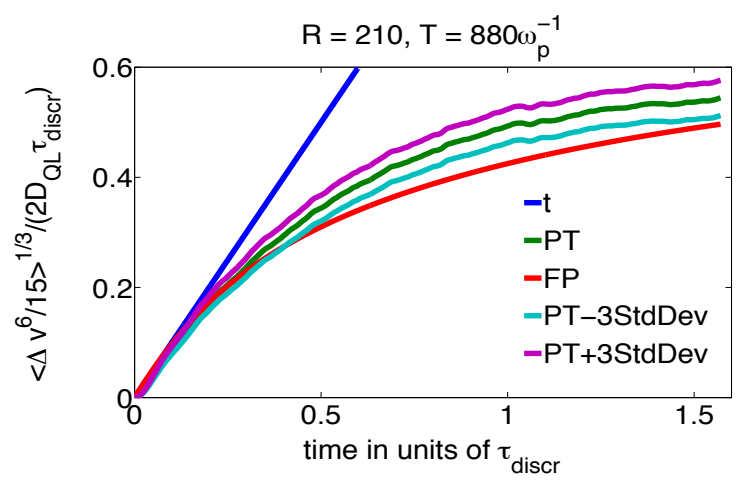

(c) $\mathfrak{M}_{6}$

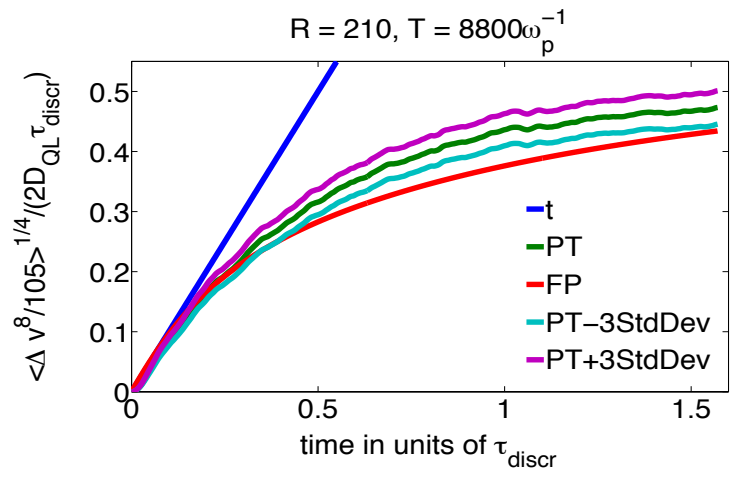

(d) $\mathfrak{M}_{8}$

Figure 15. Even moments of the particle velocity deviation. (FP) : Fokker-Planck equation. (PT) : motion of 20 test particles in $R=210$ realizations of the wave complex amplitudes $\zeta_{m}(T)$. 


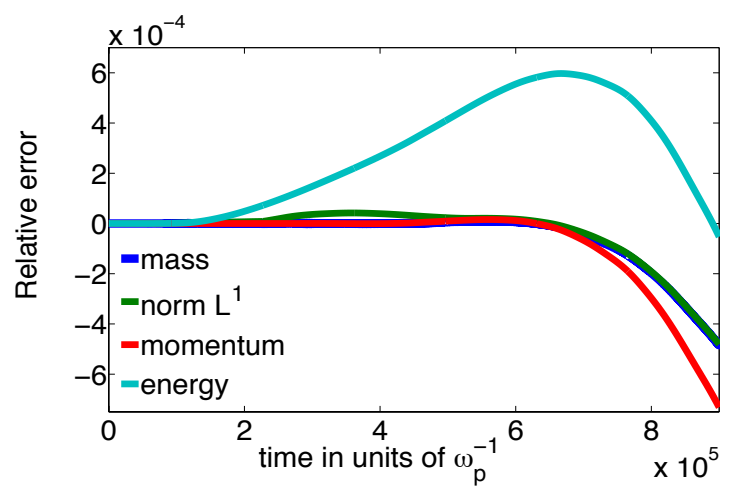

Figure 16. Relative error on conservation laws. The error on mass is almost equal to the $L^{1}$ error.

nonlinear regimes.

Since the initial distribution function is affine in velocity, the Landau growth rate of the waves varies quadratically with the velocity. From figure 18-(a), we observe a first regime from time $t=0$ to $t=1250 \omega_{\mathrm{p}}^{-1}$ where the logarithm of the waves total energy appears to vary linearly with time. More significantly, as the growth rates depend on velocity, figure 18-(c) indicates a linear stage, where every wave $m$ grows exponentially with its prescribed rate, up to time $t=1250 \omega_{\mathrm{p}}^{-1}$.

We next observe a second regime for time $t>1250 \omega_{\mathrm{p}}^{-1}$, where the faster waves no longer grow at the initial Landau rate, which indicates mode coupling processes (INL regime), followed by a nonlinear saturation regime from time $t=1.5 \times 10^{5} \omega_{\mathrm{p}}^{-1}$ to time $t=9 \times 10^{5} \omega_{\mathrm{p}}^{-1}$ at which the plateau regime is settled (see figures 18-(b) and 19).

\subsection{Transition from stochastic to chaotic diffusion}

In figures 19-(c) and (d) we observe that the space-averaged distribution function evolves from the triangular shape to the plateau one between times $t=5.4 \times 10^{4} \omega_{\mathrm{p}}^{-1}$ and $t=9 \times 10^{5} \omega_{\mathrm{p}}^{-1}$. Simultaneously, during the same time period, the growth rates of the waves fall from a strictly positive value to a null one (see figures 19-(a) and (b)). In contrast to the hyperbolic case where the saturation of all waves and the vanishing of their growth rate $\gamma$ occurred around the same time, here we observe the propagation of a front going from high velocity values to smaller ones. This front displays a hyperboliclike profile in time (see figure 19-(a)), due to the $v^{2}$ variation of $\gamma_{\mathrm{L}}$, making slower waves grow more slowly. The plateau regime is well settled at time $t=9 \times 10^{5} \omega_{\mathrm{p}}^{-1}$.

For this trianglar initial profile, the self-consistent wave spectrum is obtained by averaging over 13 realizations of the same Vlasov-wave test case where random initial phases are drawn independently from a uniform distribution on the circle. From figures 20 and 22, in the plateau regime we observe a rather good agreement of the QL prediction for the wave intensity spectrum (33) and for the diffusion coefficient with the ones obtained by self-consistent simulations of the Vlasov-wave model. These results 


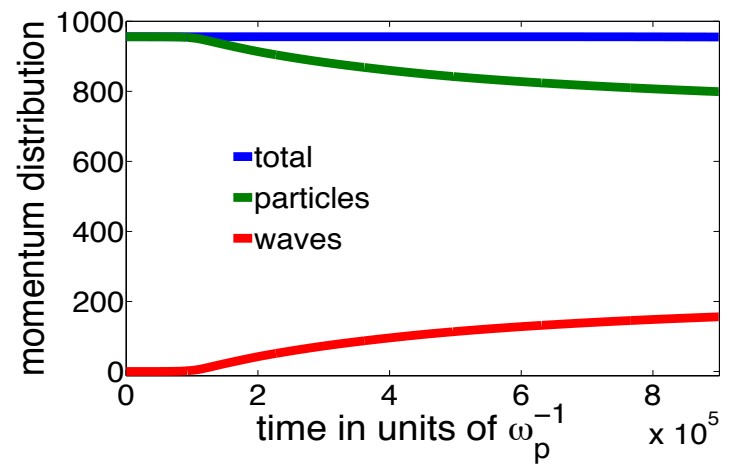

(a) Momentum sharing

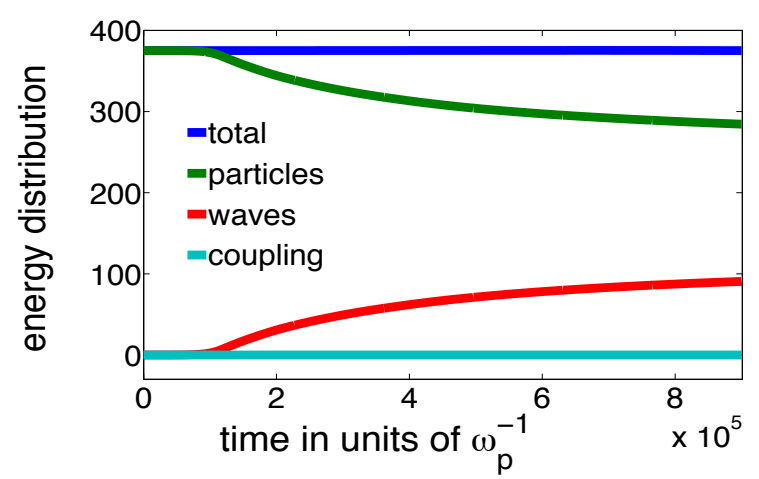

(b) Energy sharing

Figure 17. Distribution of momentum and energy.

yield again some support in favour of the validity of the quasilinear theory. The relative lack of accuracy of results presented in figures 20-(a) and 22-(a) is due to our rough statistics with only 13 realizations. However figures 20-(b) and 22-(b) give an idea of what figures 20-(a) and 22-(a) would approach if we would consider a larger statistical ensemble.

In order to assess whether regime SNL, where strong chaotic diffusion holds, has been reached at the end of the simulation, we measure the parameters $K_{\mathrm{D}}, s_{\text {ov }}$ (or $\mathcal{B}^{-1}$ ) and $\mu$. From figures 24 we notice that at the middle of the phase velocity range of the wave spectrum, i.e. for $v_{\mathrm{c}}=\left(u_{0}+u_{1}\right) / 2 \simeq 0.57$, and at time $T=9 \times 10^{5} \omega_{\mathrm{p}}^{-1}$ in the plateau regime, we have $\left\langle s_{\text {ov }}\right\rangle_{R}\left(T, v_{\mathrm{c}}\right) \simeq 15,\left\langle K_{\mathrm{D}}^{-1}\right\rangle_{R}\left(T, v_{\mathrm{c}}\right) \simeq 112,\langle\mu\rangle_{R}\left(T, v_{\mathrm{c}}\right) \simeq 1.16 \times 10^{5}$ and $\left\langle\Delta v_{\mathrm{D}}\right\rangle_{R}\left(T, v_{\mathrm{c}}\right) \simeq 10^{-2}$ which corresponds to a range of wave velocities about 100 times as wide as the velocity diffusion width. These parameter values confirm that the system is in regime SNL of strong chaotic diffusion.

Since the plateau regime is well settled at time $t=9 \times 10^{5} \omega_{\mathrm{p}}^{-1}$ (see figures 19), the amplitude spectrum variations are sufficiently small and thus negligible (see figure 25). We may again consider that the self-consistent dynamics can be approximated by the non-self-consistent one. In order that QL estimates should hold in regime SNL, the wave spectrum has to be smooth enough over the Dupree width, and the phases should 


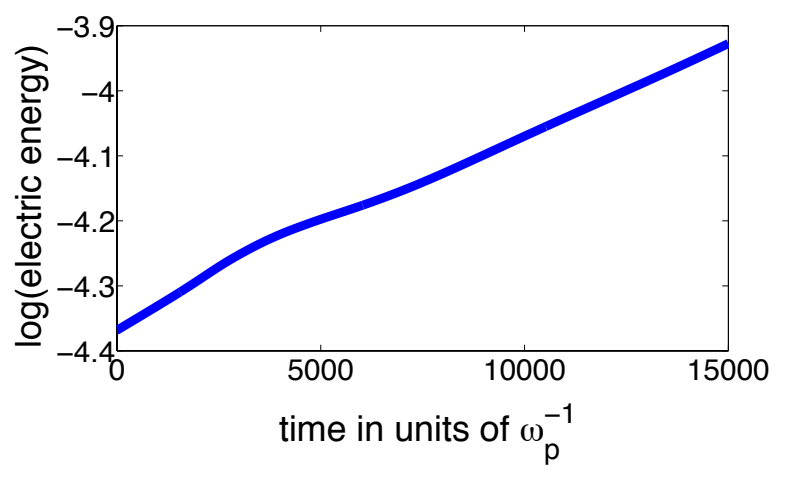

(a) Electric energy

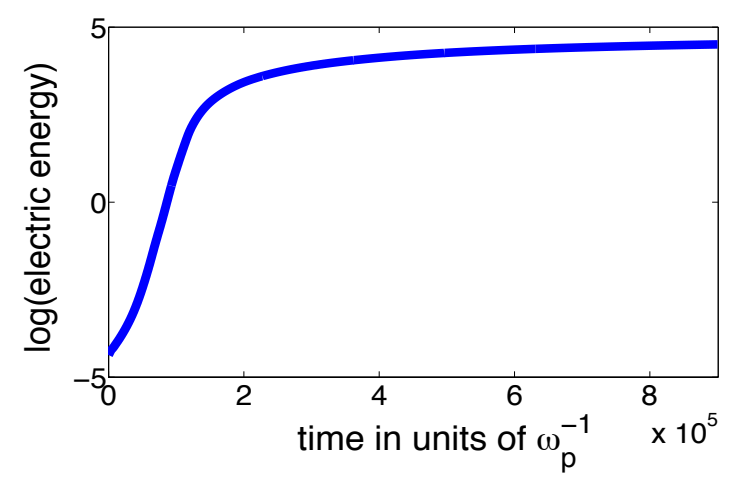

(b) Electric energy

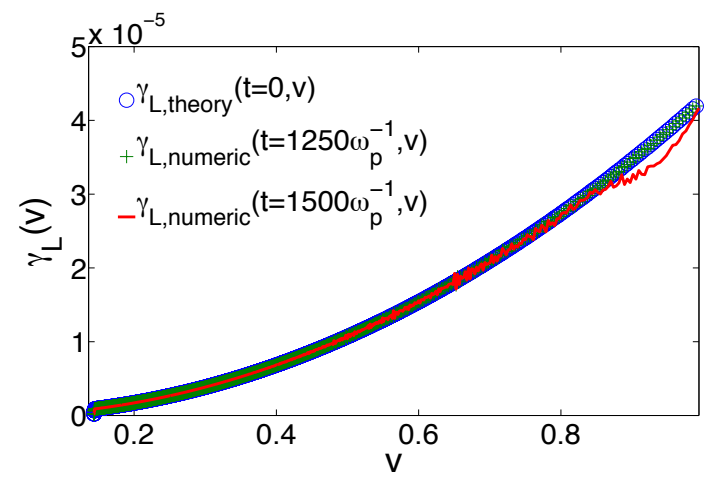

(c) Growth rates

Figure 18. Growth of waves in linear and INL regimes.

be random. In figure 21-(a) (resp. figure 23-(a)) we observe for a single realization that the velocity dependence of the wave spectrum intensity (resp. diffusion coefficient) is very noisy, but when averaged over a velocity width corresponding to a floating average (31) with $L_{\text {avg }}=5$, wave spectrum intensities (resp. diffusion coefficients) describe a smooth function with respect to velocity, see figure 21-(b) (resp. figure 23-(b)). This is a conservative test, since at the middle of the phase velocity range of the wave spectrum, the ratio $L_{\mathrm{avg}, \mathrm{D}}(t, v)=\Delta v_{\mathrm{D}} / \Delta v_{\varphi}=\mathcal{B}^{-1}$ is about 12 (see figures 24). Figures 20 and 22 


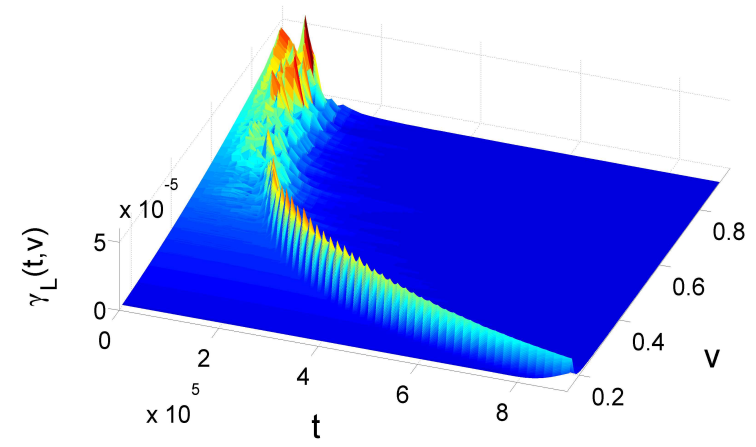

(a) $\gamma_{\mathrm{L}}(t, v)$

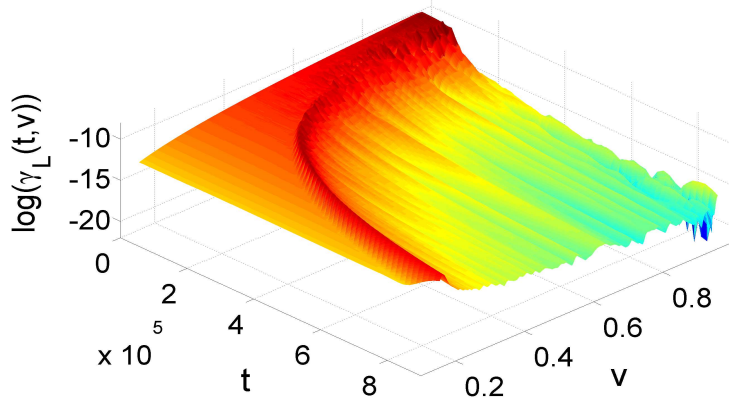

(b) $\log \left(\gamma_{\mathrm{L}}(t, v)\right)$

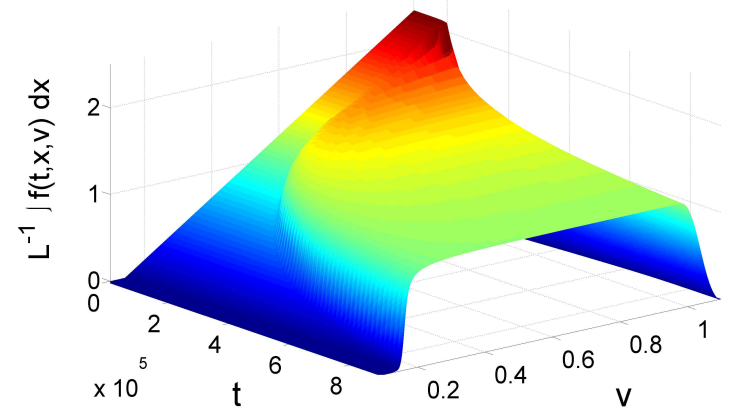

(c) $\frac{1}{L} \int f(t, x, v) \mathrm{d} x$

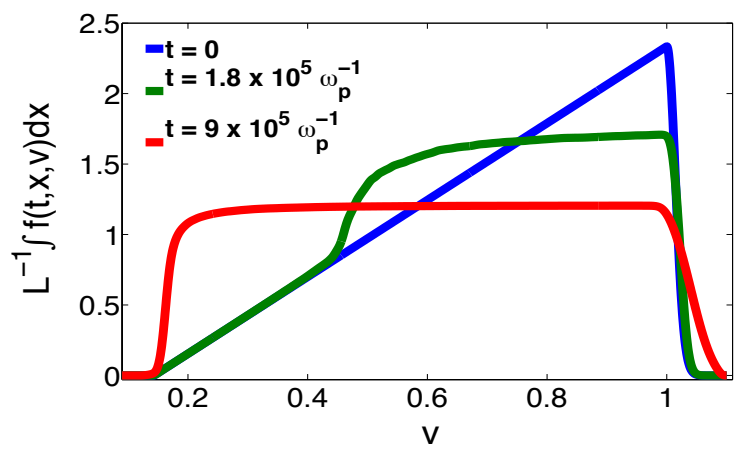

(d) $\frac{1}{L} \int f(t, x, v) \mathrm{d} x$

Figure 19. Time evolution of the Landau growth rates and space-averaged distribution function. 


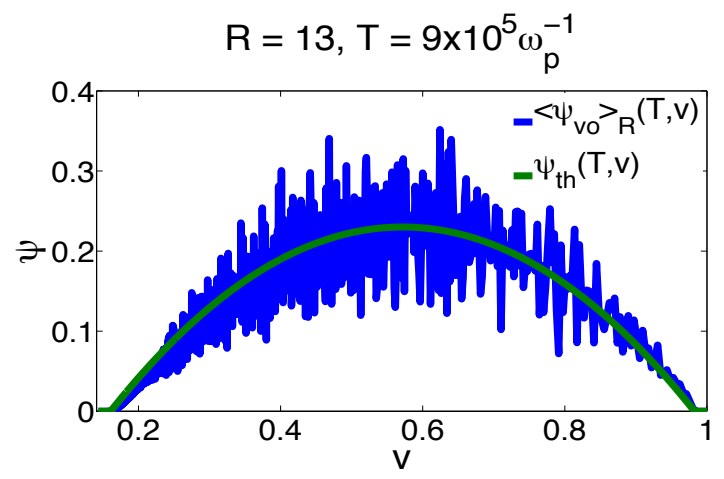

(a) $\langle\psi\rangle_{R}(T, v)$ vs. $\psi_{\text {th }}(T, v)$

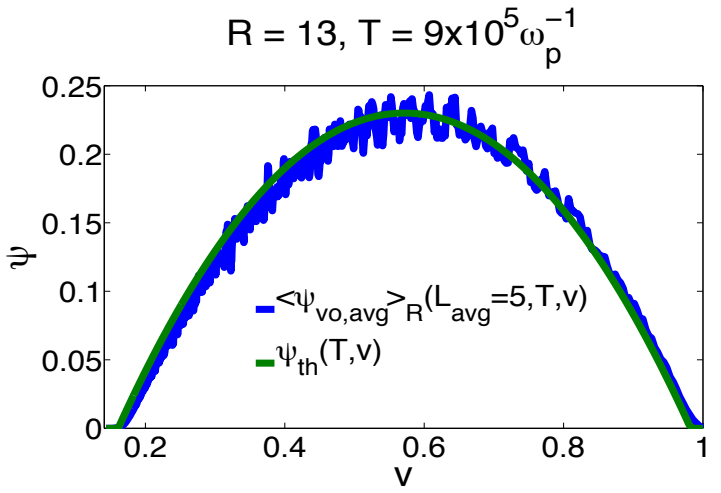

(b) $\left\langle\psi_{\text {avg }}\right\rangle_{R}(T, v)$ vs. $\psi_{\text {th }}(T, v)$

Figure 20. Ensemble averaged wave power spectrum.

show that the average amplitudes of the waves and corresponding diffusion coefficients are quite smooth with respect to their velocity. The spectrum generated by our selfconsistent simulations thus fulfils the first hypothesis about the absence of holes larger than the order of a width $\Delta v_{\mathrm{D}}$.

From figures 27 we observe that the final phases are drawn independently from a uniform distribution on the circle as was the case at initial time, which satisfies the second requirement. Even for a single, typical run, with random initial phases uniformly distributed on the circle, figure 26 shows roughly that the final phases of waves with nearby velocities are uncorrelated. Therefore the wave spectrum fulfils the assumptions which lead to QL estimates [21].

Again we test the validity of the QL diffusion model with the direct integration of the particle equations of motion. We follow $N=100$ test particles in $R=13$ realizations of the wave data $\left(\zeta_{m}\right)$ at $T=9 \times 10^{5} \omega_{\mathrm{p}}^{-1}$ and compare even moments of their velocity distribution with the corresponding moments of the solution to the FokkerPlanck equation with QL diffusion coefficient. Figures 28 show that the agreement is very good up to times exceeding the discretisation time $\tau_{\text {discr }}$. This is much longer than for the hyperbolic initial particle velocity distribution, clearly thanks to the wave spectrum here allowing for more waves to act incoherently on the particles. The Fokker- 


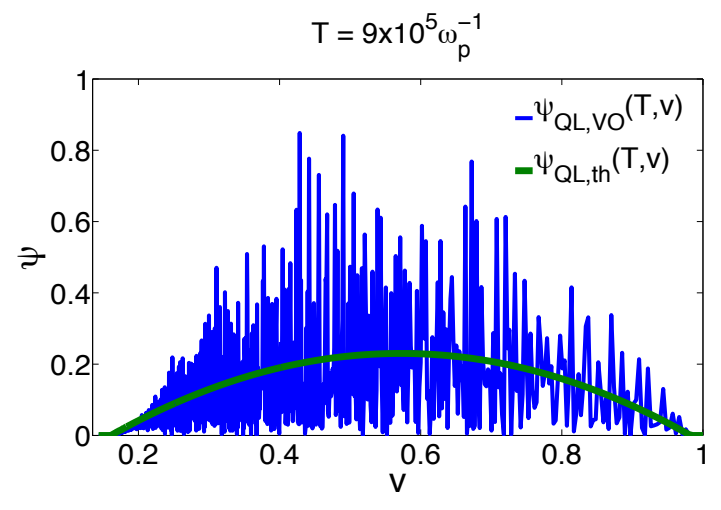

(a) $\psi(T, v)$ vs. $\psi_{\text {th }}(T, v)$

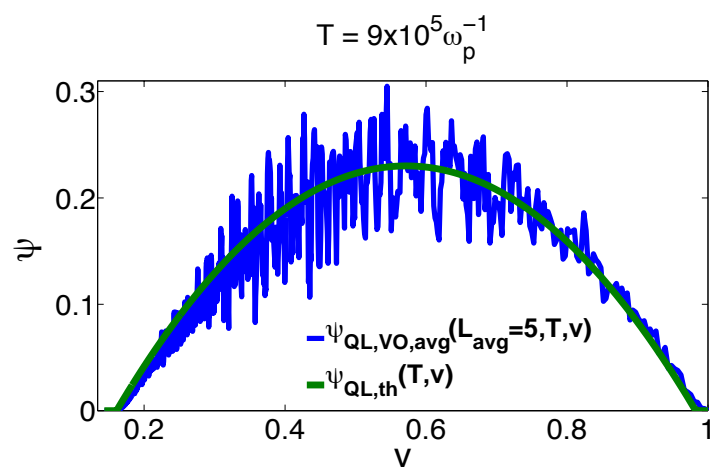

(b) $\psi_{\text {avg }}(T, v)$ vs. $\psi_{\text {th }}(T, v)$

Figure 21. Typical wave power spectrum for a single realization.

Planck equation (1) definitely accounts for the motion of particles in the plateau wave spectrum.

\section{Conclusion}

The validity of quasilinear theory for describing the weak warm beam-plasma instability in the chaotic saturation regime was considered in this work both analytically and numerically. It was shown intuitively and analytically that there is no mode coupling in the saturation regime of the instability, where a plateau is present in the tail of the particle distribution function. This contradicts previous analytical works attempting to prove the validity of quasilinear theory in the strongly nonlinear regime of the weak warm beam-plasma instability $[42,43,28,21]$ and the "turbulent trapping" Ansatz aiming at the contrary [40].

Then this work described a series of self-consistent simulations of the weak warm beam-plasma instability within the Vlasov-wave description. They confirmed the occurrence of an enhanced growth rate in the intermediate nonlinear regime already shown in [15], and consistent with the perturbative calculation of [38]. This enhancement contradicts the analytical arguments for validity of quasilinear theory in [51]. However 


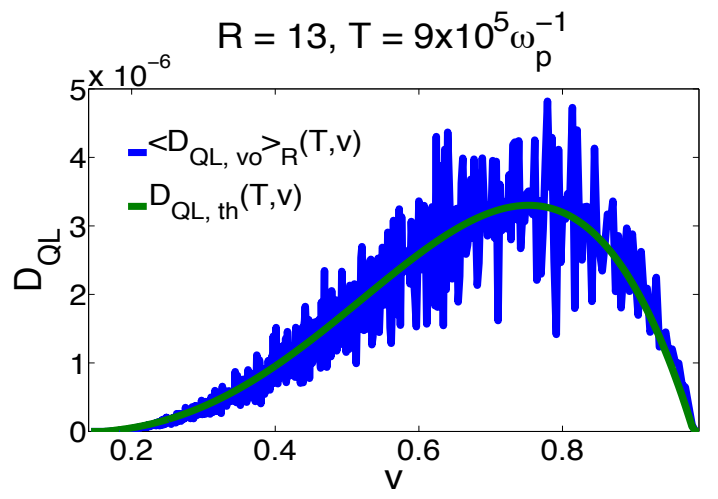

(a) $\left\langle D_{\mathrm{QL}}\right\rangle_{R}(T, v)$ vs. $D_{\mathrm{QL}, \mathrm{th}}(T, v)$

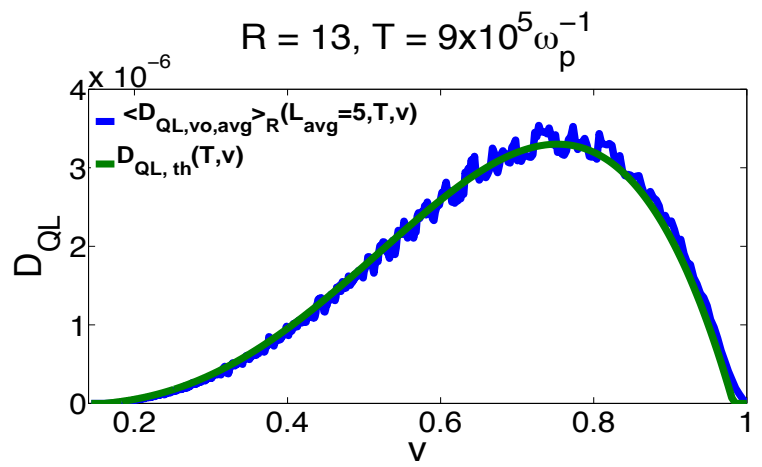

(b) $\left\langle D_{\mathrm{QL}, \text { avg }}\right\rangle_{R}(T, v)$ vs. $D_{\mathrm{QL}, \mathrm{th}}(T, v)$

Figure 22. Ensemble averaged quasilinear diffusion coefficient.

the wave growth rate was shown to relax toward its quasilinear value when going into regime SNL. They also showed that QL theory remains valid in the strong chaotic diffusion regime (regime SNL). In particular, the QL diffusive approximation for particle motion in the saturated wave spectrum was shown to hold over time scales up to the order of the beam spreading through the wave spectrum. This work also confirmed the relevance of the diffusive model for particle motion in regime SNL by comparing the statistics of direct integration of the equations of motion with the statistics of the diffusion model. Thus both the mode growth rates in regime INL and the particle diffusion process in the plateau regime SNL remain rather close to the QL predictions.

In conclusion, the main argument for a possible renormalization of the quasilinear growth rate and diffusion coefficient in the strongly nonlinear regime of the weak warm beam-plasma instability was discarded on an analytical and intuitive basis, and a thorough numerical simulation was consistent with the quasilinear prediction in this regime. Together with the refutation of other analytical works, this brings an important milestone to the quasilinear controversy. However a rigorous and intuitive description of the whole saturation is still awaited. 


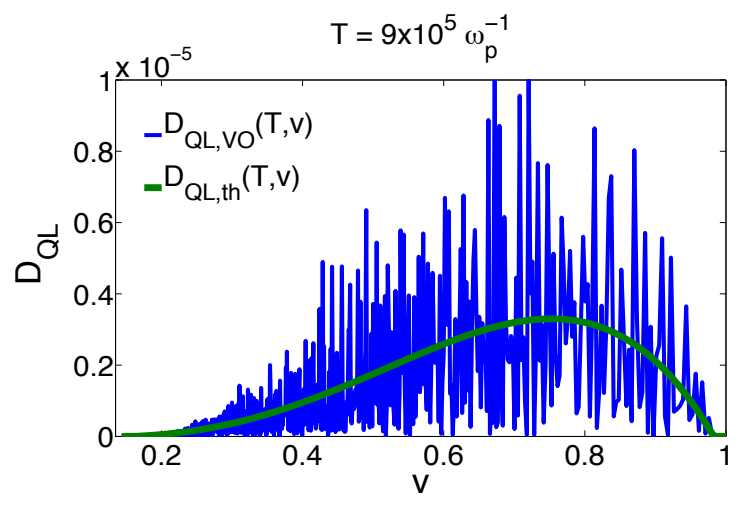

(a) $D_{\mathrm{QL}}(T, v)$ vs. $D_{\mathrm{QL}, \mathrm{th}}(T, v)$

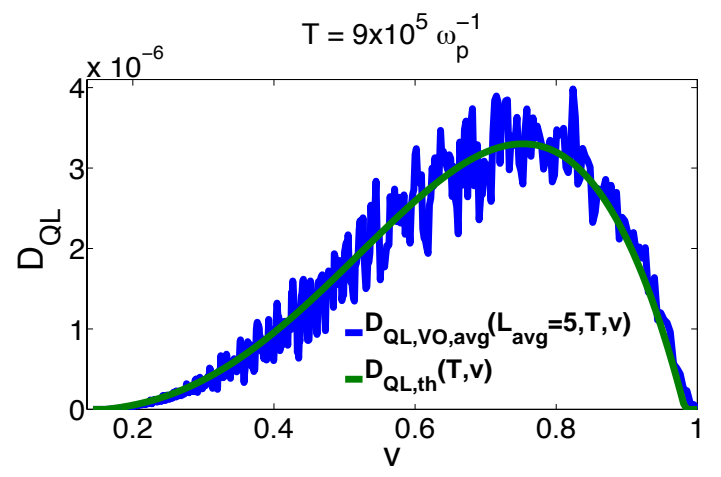

(b) $D_{\mathrm{QL}, \text { avg }}(T, v)$ vs. $D_{\mathrm{QL}, \mathrm{th}}(T, v)$

Figure 23. Typical quasilinear diffusion coefficient for a single realization.

\section{Acknowledgement}

This work was granted access to the HPC ressources of IDRIS (IBM-Power6 computer) under allocation i-2010-056002 made by GENCI (Grand Equipement National de Calcul Intensif). It is a pleasure to acknowledge D. Bénisti's comments, which were an incentive for revisiting the analytical part of the paper. YE is grateful to N. Dubuit for technical tips.

\section{Appendix A. Time scales for diffusion and velocity resonance boxes}

This appendix makes more precise the discussion in section 1 about diffusive transport in the dynamics defined by Hamiltonian (9). Physically, the larger the velocity of a particle in a wave frame, the smaller the influence of the wave on the particle. Even in the chaotic regime, strongly enough non-resonant waves may be treated through perturbations theory $[4,5,6,21]$. Put briefly, the interaction is local in velocity.

This idea of locality was already present in the resonance broadening concept introduced by Dupree [17]. A more complete analysis [21, 4, 5, 6] shows that for strong resonance overlap $\left(s_{\text {ov }} \gg 1\right.$ or $\mathcal{B} \ll 1$ ), only waves with a phase velocity within the 


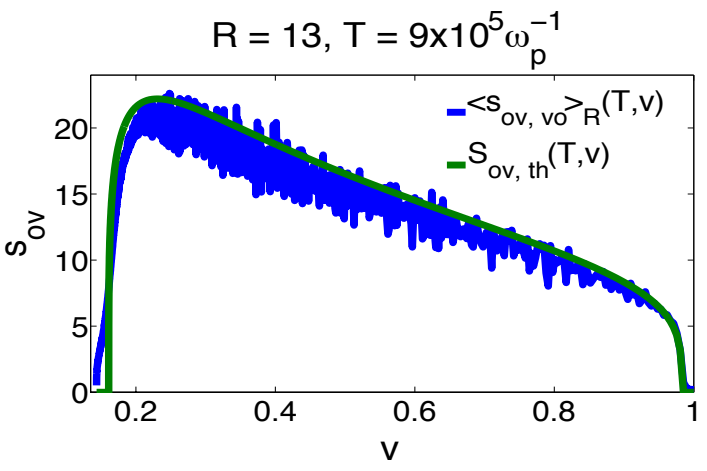

(a) $\left\langle s_{\text {ov }}\right\rangle_{R}(T, v)$ vs. $s_{\text {ov,th }}(T, v)$

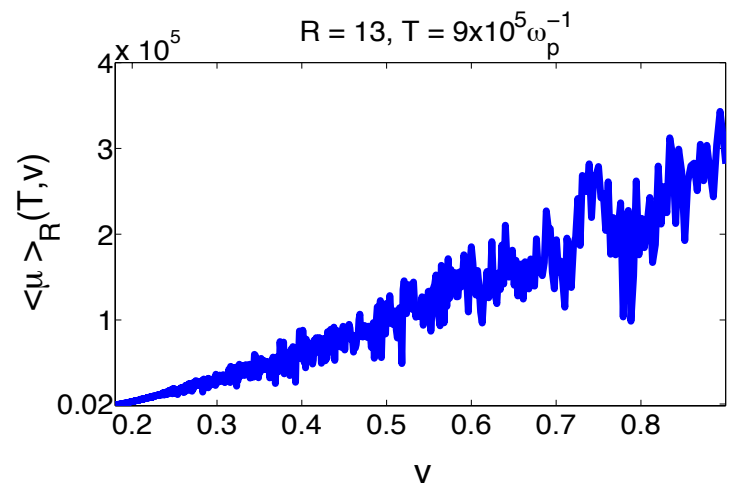

(b) $\langle\mu\rangle_{R}(T, v)$

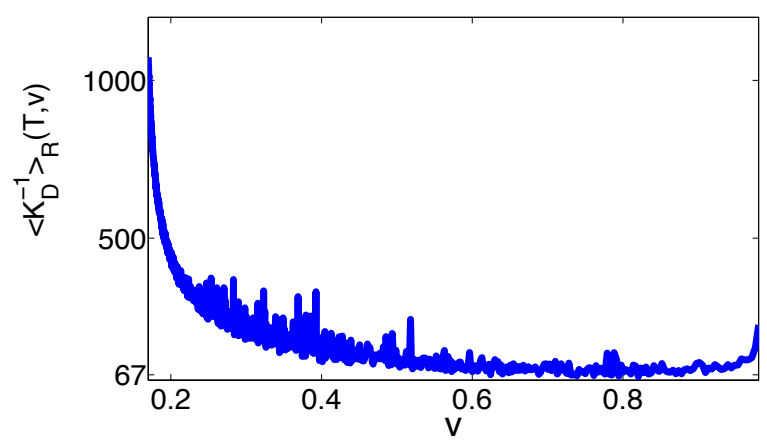

(c) $\left\langle K_{\mathrm{D}}^{-1}\right\rangle_{R}(T, v)$

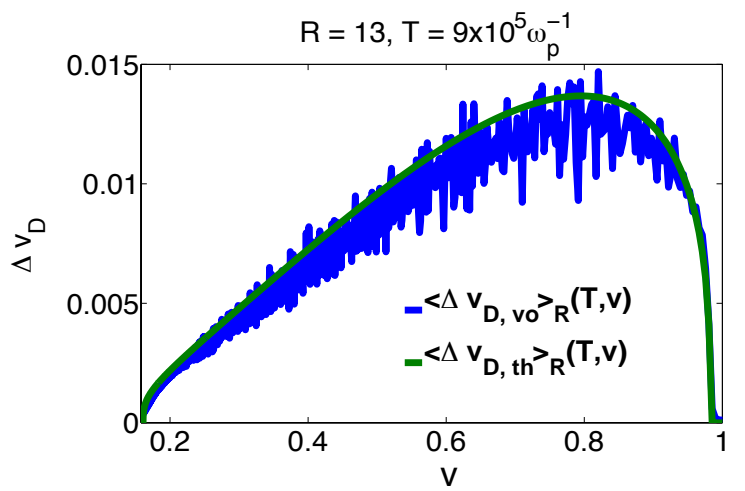

(d) $\left\langle\Delta v_{\mathrm{D}}\right\rangle_{R}(T, v)$ vs. $\Delta v_{\mathrm{D}, \text { th }}(T, v)$

Figure 24. Physical parameters. 


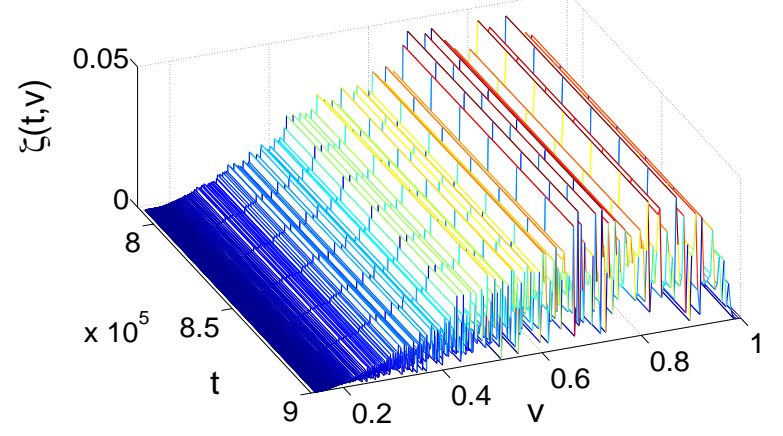

Figure 25. Time evolution of wave intensity spectrum $|\zeta(t, v)|$, from time $t=$ $8 \times 10^{5} \omega_{p}^{-1}$ to time $t=9 \times 10^{5} \omega_{p}^{-1}$.

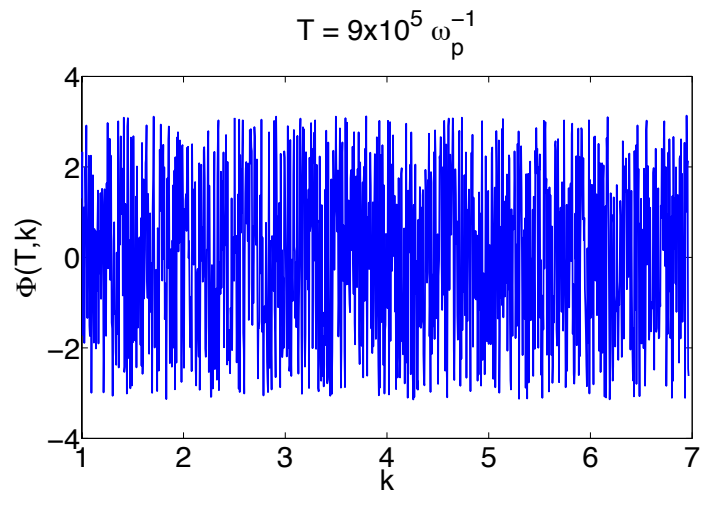

Figure 26. Typical final phase of the wave spectrum for a single realization.

range (called resonance box) centered on the particle velocity of width $2 \Delta v_{\text {box }}$ with $\Delta v_{\text {box }} \simeq 4.6 \Delta v_{\mathrm{D}}$ contribute to chaotic transport of the particle. Note that parameter $\mathcal{B}$ can be rewritten as the ratio $\mathcal{B}=4.6 \Delta v_{\varphi} / \Delta v_{\text {box }}$, so that $4.6 \mathcal{B}^{-1}$ can be viewed as the typical number of waves in a resonance box. We can also introduce the parameter $K_{\text {box }}=\Delta v_{\text {box }} / \Delta v_{\text {spec }}$ which is equivalent to the Kubo number $K_{\mathrm{D}}$ and is associated with the competition between stochastic and deterministic time-scales in stochastic processes. Finally we can introduce the time $\tau_{\text {box }}=\left(2 \Delta v_{\text {box }}\right)^{2} /\left(2 D_{\mathrm{QL}}\right) \simeq 11 \tau_{\text {spread }} \simeq 40 \tau_{\mathrm{D}}$ which is the typical time it takes for a resonant particle to wander through a resonance box.

Since large scale chaos makes the orbit unconfined in velocity, it visits a sequence of resonance boxes of width $2 \Delta v_{\text {box }}$, where the wave random phases are independent. Hence, the velocity undergoes a series of independent increments, which lead to a diffusion by a central limit effect. Therefore if we note $N_{\text {box }}=\Delta v_{\text {spec }} /\left(2 \Delta v_{\text {box }}\right) \simeq$ $\left(9 K_{\mathrm{D}}\right)^{-1}$, we see that the regime of strong chaotic diffusion, regime SNL, is in fact characterized by the conditions $\mu \gg 1$ (slow wave evolution), $\mathcal{B} \ll 1$ (strong chaos by strong resonance overlap) and $N_{\text {box }} \gtrsim 3$ (wide diffusion range). It is also important to notice that a random initial position alone does not impose a Gaussian statistics to the 


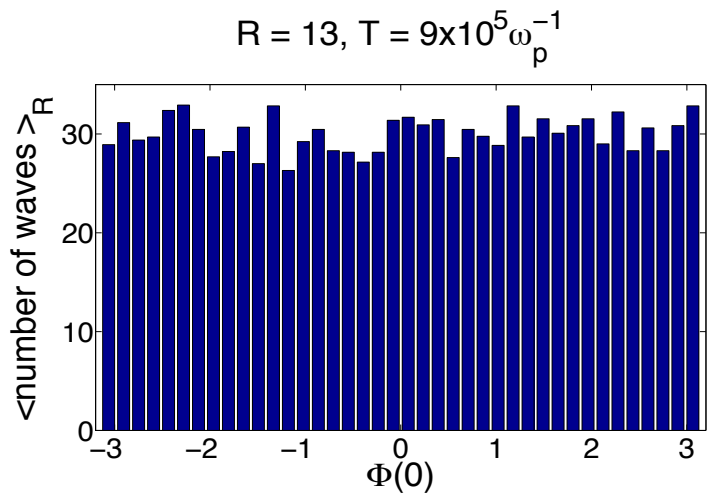

(a) Initial phase

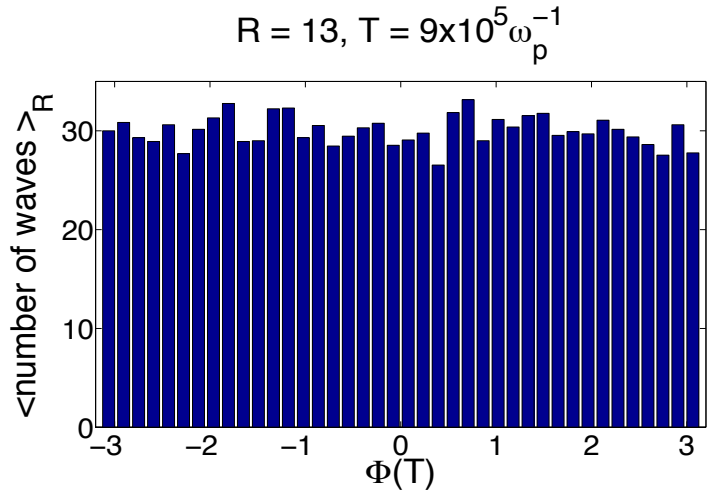

(b) Final phase

Figure 27. Distribution of the phases of the wave spectrum.

velocity: wave phase randomness is essential $[4,5,6,21]$.

The wave phase randomness of course underlies the original QL theory, as it essentially implies that, for short times, the particle is subject to an uncorrelated force field, physically a white noise. Considering this picture as a perturbation to ballistic motion of the particle leads first to the traditional requirement $\left\langle k^{2} \Delta x^{2}\right\rangle \lesssim 4 \pi^{2}$ of the original QL estimates, which applies only for $t \lesssim \tau_{\text {spread }}$ and forces the orbit to have a weak dependence on all $M \gg 2$ phases simultaneously. However, if the orbit merely has a weak dependence on any $N_{\varphi}=2$ phases, all other phases been fixed (which is less stringent than the previous condition of type $N_{\varphi}=M$ ), formal integration of the equation of motion reveals that $\left\langle\Delta v^{2}\right\rangle$ take its $\mathrm{QL}$ value over times beyond $\tau_{\text {spread }}$ $[4,5,6,21]$, as is recalled now.

Actually, linear theory enables one to compute rigorously the small variation of the orbit when any two phases are varied [27, 28, 29, 21]. Indeed, since the particle initial position is the same for all realizations of the wave phases, the initial mismatch between two realizations of the orbit is initially small and can also be computed by linear theory. This calculation shows that $D=D_{\mathrm{QL}}$ for $t \lesssim \tau_{\mathrm{QL}}=\tau_{\text {spread }}|\ln \mathcal{B}|$ for strong resonance overlap (and in particular for a continuous spectrum $\mathcal{B} \rightarrow 0$ ). In this regime, $\tau_{\mathrm{QL}} \gg \tau_{\text {spread }}$, which means that the QL estimate for the diffusion coefficient 


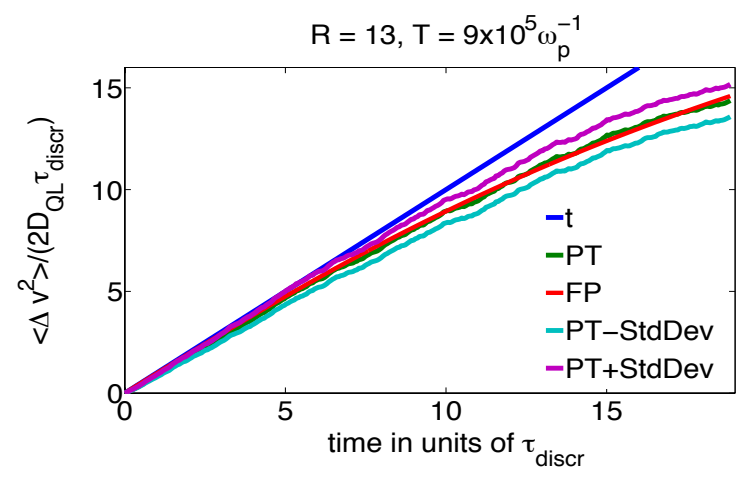

(a) $\mathfrak{M}_{2}$

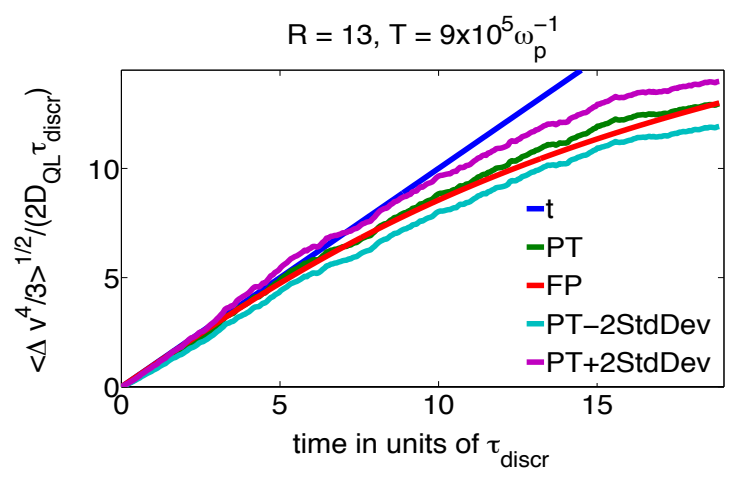

(b) $\mathfrak{M}_{4}$

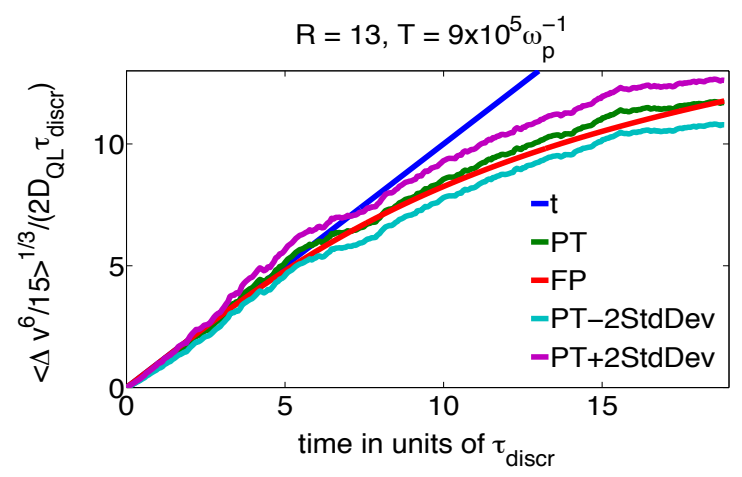

(c) $\mathfrak{M}_{6}$

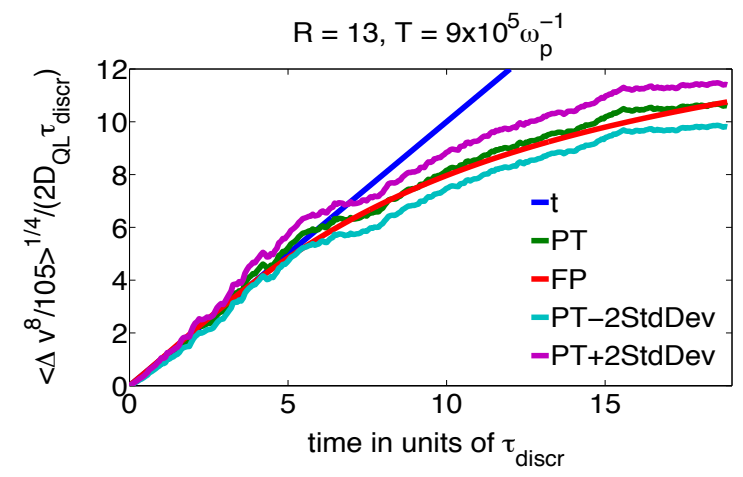

(d) $\mathfrak{M}_{8}$

Figure 28. Even moments of the particle velocity deviation. (FP) : Fokker-Planck equation. (PT) : motion of 100 test particles in $R=13$ realizations of the wave amplitudes. 
is correct for times much larger than $\tau_{\text {spread }}$. Moreover as $\tau_{\mathrm{QL}} \gg \tau_{\text {box }} \gtrsim \tau_{\text {spread }}$ for $\mathcal{B} \ll 1$, there is an overlap between the initial non-chaotic QL regime and the final chaotic diffusion one. This confirms that a small value of $\mathcal{B}$ (strong resonance overlap) is required for the transition from regime $\mathrm{L}$ to regime SNL.

The time scale over which QL estimates hold can still be stretched significantly using a different viewpoint. Indeed, if the waves are random, their superposition is essentially an approximation to white noise for times $t>\tau_{\mathrm{ac}} \sim\left(k \Delta v_{\mathrm{spec}}\right)^{-1}$. However, this white noise is approximated using a discrete wave spectrum, which allows for echo at a period $2 \pi \tau_{\text {discr }}=2 \pi /\left(k \Delta v_{\varphi}\right)$. Therefore, one can also prove that for a time span $0<t \lesssim \tau_{\text {discr }}$ the particle velocity essentially undergoes a brownian diffusion. For longer times, the strong overlap limit $\mathcal{B} \rightarrow 0$ implies that the particle motion "forgets" its initial sampling of the wave spectrum, which enables one to prove the validity of $\mathrm{QL}$ estimates for large $t / \tau_{\text {discr }}$ through an ergodic theorem using martingale properties of the dynamics $[19,20,23]$.

\section{Appendix B. Mode-mode coupling in the wide plateau regime}

In this appendix we calculate the modulation of the particle velocity near the plateau edge and the resulting feedback on waves, considered in section 2.2. This problem can be treated perturbatively, as the edge particles move quite regularly, with velocity $v(t)>v_{\mathrm{b}}$ or $v(t)<v_{\mathrm{a}}$, and are coupled to waves with phase velocity $v_{\varphi} \in\left[u_{0}, u_{1}\right] \subset$ $\left[v_{\mathrm{a}}, v_{\mathrm{b}}\right]$. Therefore the particles stay away from resonances ; typically one finds this $\Delta v_{\text {edge }} \approx v_{\mathrm{b}}-u_{1} \sim\left(\varepsilon \beta \zeta_{*}\right)^{c}$ with $c=\frac{1}{2}$ for a single wave model and $c=\frac{2}{3}$ for a resonance box scaling [5].

The water bag distribution, with height $f_{0}$, is fully described by its two boundaries, where by assumptions the particles move regularly with velocities near $v_{0}$ and $v_{1}$. The $M$ waves modulate these boundaries, so that at time $t$ their equations read $v=v_{-}(t, x)=v_{0}+\delta v_{-}(t, x)$ and $v=v_{+}(t, x)=v_{1}+\delta v_{+}(t, x)$. Here $x$ is a mere label on the position axis, and $f_{0}=1 /\left(v_{1}-v_{0}\right)$. The wave deformation $\theta_{m}=\zeta_{m}(t)-\zeta_{m}(0)$, which must be small over the time scale of interest, is generated by

$$
\begin{aligned}
\dot{\theta}_{m}(t) & =\varepsilon \mathrm{i} \frac{\beta_{m}}{k_{m} L} \int_{0}^{L} \int_{v_{0}+\delta v_{-}(t, x)}^{v_{1}+\delta v_{+}(t, x)} \mathrm{e}^{-\mathrm{i} k_{m} x+\mathrm{i} \omega_{m} t} f_{0} \mathrm{~d} v \mathrm{~d} x \\
& =\varepsilon \mathrm{i} \frac{\beta_{m}}{k_{m} L} \int_{0}^{L} \frac{v_{1}+\delta v_{+}(t, x)-v_{0}-\delta v_{-}(t, x)}{v_{1}-v_{0}} \mathrm{e}^{-\mathrm{i} k_{m} x+\mathrm{i} \omega_{m} t} \mathrm{~d} x \\
& =\varepsilon \mathrm{i} \frac{\beta_{m}}{k_{m} L} \int_{0}^{L} \frac{\delta v_{+}(t, x)-\delta v_{-}(t, x)}{v_{1}-v_{0}} \mathrm{e}^{-\mathrm{i} k_{m} x+\mathrm{i} \omega_{m} t} \mathrm{~d} x
\end{aligned}
$$

which is $\mathrm{O}\left(\varepsilon \beta_{m} k_{m}^{-1} L^{-1} \int_{0}^{L}\left(\left|\delta v_{+}\right|+\left|\delta v_{-}\right|\right) \mathrm{d} x / \Delta v_{\text {plat }}\right)$. The dominant contribution to (B.1) in an $\varepsilon$ expansion is thus determined by the lowest order approximation to the $k_{m}$ Fourier mode of the modulations $\delta v_{ \pm}(t, x)$, which we now estimate.

Since particles on the boundaries follow characteristics of the Vlasov equation, we

now describe the faster boundary in terms of particle positions $x_{0}$ at time $t_{0}$. We 
compute the correction $s(t)=x(t)-x_{0}-v_{1} t, w(t)=v(t)-v_{1}$ by integrating the characteristic equations in the form

$$
\begin{aligned}
\dot{s} & =w, \\
\dot{w} & =\operatorname{Re} \sum_{m} \mathrm{i} \varepsilon \beta_{m} \zeta_{m}(t) \mathrm{e}^{\mathrm{i}\left(k_{m} x-\omega_{m} t\right)} \\
& =\sum_{m} \frac{\mathrm{i}}{2} \varepsilon\left[\alpha_{m} \mathrm{e}^{\mathrm{i}\left(k_{m} s+\Omega_{m} t\right)}-\alpha_{m}^{*} \mathrm{e}^{-\mathrm{i}\left(k_{m} s+\Omega_{m} t\right)}\right]
\end{aligned}
$$

where $\Omega_{m}=k_{m} v_{1}-\omega_{m}$ and $\alpha_{m}=\beta_{m} \zeta_{m} \mathrm{e}^{\mathrm{i} k_{m} x_{0}}$. To dominant order in $\varepsilon$, we may treat $\zeta_{m}(t)$ as a constant, $\zeta_{m}$.

By the adiabatic assumption, $s$ must be uniformly small over the time interval of interest, and we Taylor expand the exponentials with respect to $s(t)=\sum_{j=1}^{3} \varepsilon^{j} s_{j}+\mathrm{O}\left(\varepsilon^{4}\right)$, so that

$$
s_{1}(t)=S_{1}+W_{1} t-\sum_{\ell} \frac{\mathrm{i}}{2} \Omega_{\ell}^{-2}\left(\alpha_{\ell} \mathrm{e}^{\mathrm{i} \Omega_{\ell} t}-\alpha_{\ell}^{*} \mathrm{e}^{-\mathrm{i} \Omega_{\ell} t}\right)
$$

where constants $S_{1}, W_{1}$ are arbitrary. They depend on $x_{0}$, but the KAM requirement for the boundary mean velocity being $v_{1}$ is met by letting both of them vanish $\left(W_{1}=0\right.$ eliminates secular behaviour, and $S_{1}=0$ ensures that the boundary is labeled uniformly by $x_{0}$, on the average). This in turn leads to the second order approximation,

$$
\ddot{s}_{2}=\sum_{p} \frac{\mathrm{i}}{2}\left[\alpha_{p} \mathrm{e}^{\mathrm{i} \Omega_{p} t}+\alpha_{p}^{*} \mathrm{e}^{-\mathrm{i} \Omega_{p} t}\right] \mathrm{i} k_{p} s_{1}
$$

so that

$$
\begin{aligned}
s_{2}(t)=S_{2}+W_{2} t+ & \sum_{p} \sum_{\ell} \frac{\mathrm{i}}{4} \Omega_{\ell}^{-2} k_{p} \\
& {\left[\left(1-\delta_{\ell p}\right)\left(\Omega_{\ell}-\Omega_{p}\right)^{-2}\left(\alpha_{p}^{*} \alpha_{\ell} \mathrm{e}^{\mathrm{i}\left(\Omega_{\ell}-\Omega_{p}\right) t}-\alpha_{p} \alpha_{\ell}^{*} \mathrm{e}^{\mathrm{i}\left(\Omega_{p}-\Omega_{\ell}\right) t}\right)\right.} \\
& \left.+\left(\Omega_{\ell}+\Omega_{p}\right)^{-2}\left(\alpha_{p} \alpha_{\ell} \mathrm{e}^{\mathrm{i}\left(\Omega_{\ell}+\Omega_{p}\right) t}-\alpha_{p}^{*} \alpha_{\ell}^{*} \mathrm{e}^{-\mathrm{i}\left(\Omega_{\ell}+\Omega_{p}\right) t}\right)\right]
\end{aligned}
$$

where again we set $W_{2}=0, S_{2}=0$, and $\delta_{\ell p}$ is the Kronecker symbol. The equation for the third order velocity correction follows,

$$
\dot{w}_{3}=\sum_{q} \frac{\mathrm{i}}{2}\left[\alpha_{q} \mathrm{e}^{\mathrm{i} \Omega_{q} t}\left(\mathrm{i} k_{q} s_{2}-\frac{1}{2} k_{q}^{2} s_{1}^{2}\right)+\alpha_{q}^{*} \mathrm{e}^{-\mathrm{i} \Omega_{q} t}\left(\mathrm{i} k_{q} s_{2}+\frac{1}{2} k_{q}^{2} s_{1}^{2}\right)\right] .
$$

Its solution, with $W_{3}=0$ as for the lower orders, reads

$$
\begin{aligned}
w_{3}= & \frac{1}{16} \sum_{q, p, \ell} k_{q}^{2} \Omega_{p}^{-2} \Omega_{\ell}^{-2}\left(W_{q p \ell}+W_{q,-p,-\ell}+W_{-q, p,-\ell}+W_{-q,-p, \ell}\right) \\
& -\frac{1}{8} \sum_{q, p, \ell} k_{q} k_{p} \Omega_{\ell}^{-2}\left(\Omega_{p}+\Omega_{\ell}\right)^{-2}\left(W_{q p \ell}+W_{q,-p,-\ell}\right) \\
& -\frac{1}{8} \sum_{q, p, \ell} k_{q} k_{p} \Omega_{\ell}^{-2}\left(\Omega_{p}-\Omega_{\ell}\right)^{-2}\left(1-\delta_{p \ell}\right)\left(W_{-q, p,-\ell}-W_{-q,-p, \ell}\right)
\end{aligned}
$$

where

$W_{q p \ell}=\left(\Omega_{q}+\Omega_{p}+\Omega_{\ell}\right)^{-1}\left(\alpha_{q} \alpha_{p} \alpha_{\ell} \mathrm{e}^{\mathrm{i}\left(\Omega_{q}+\Omega_{p}+\Omega_{\ell}\right) t}+\alpha_{q}^{*} \alpha_{p}^{*} \alpha_{\ell}^{*} \mathrm{e}^{-\mathrm{i}\left(\Omega_{q}+\Omega_{p}+\Omega_{\ell}\right) t}\right)$ 
$W_{q,-p,-\ell}=\left(\Omega_{q}-\Omega_{p}-\Omega_{\ell}\right)^{-1}\left(\alpha_{q} \alpha_{p}^{*} \alpha_{\ell}^{*} \mathrm{e}^{\mathrm{i}\left(\Omega_{q}-\Omega_{p}-\Omega_{\ell}\right) t}+\alpha_{q}^{*} \alpha_{p} \alpha_{\ell} \mathrm{e}^{-\mathrm{i}\left(\Omega_{q}-\Omega_{p}-\Omega_{\ell}\right) t}\right)$

$W_{-q, p,-\ell}=\left(-\Omega_{q}+\Omega_{p}-\Omega_{\ell}\right)^{-1}\left(\alpha_{q}^{*} \alpha_{p} \alpha_{\ell}^{*} \mathrm{e}^{\mathrm{i}\left(-\Omega_{q}+\Omega_{p}-\Omega_{\ell}\right) t}+\alpha_{q} \alpha_{p}^{*} \alpha_{\ell} \mathrm{e}^{\mathrm{i}\left(\Omega_{q}-\Omega_{p}+\Omega_{\ell}\right) t}\right)$

$W_{-q,-p, \ell}=\left(-\Omega_{q}-\Omega_{p}+\Omega_{\ell}\right)^{-1}\left(\alpha_{q}^{*} \alpha_{p}^{*} \alpha_{\ell} \mathrm{e}^{\mathrm{i}\left(-\Omega_{q}-\Omega_{p}+\Omega_{\ell}\right) t}+\alpha_{q} \alpha_{p} \alpha_{\ell}^{*} \mathrm{e}^{\mathrm{i}\left(\Omega_{q}+\Omega_{p}-\Omega_{\ell}\right) t}\right)$

To compute the $\delta v_{+}$contribution to (B.1) we only need the $k_{m}$ spatial Fourier component in $w$, as $\delta v_{+}(t, x)=w\left(t, x_{0}\right)=w\left(t, x-v_{1} t-s\right)$. Thus, to dominant order, $\mathrm{e}^{-\mathrm{i} k_{m} x} \delta v_{+}(t, x)=\mathrm{e}^{-\mathrm{i} k_{m} x_{0}-\mathrm{i} k_{m} v_{1} t} w\left(t, x_{0}-v_{1} t\right)+\mathrm{O}\left(k_{m} s w\right)$, and in the integral this change of variable yields $\mathrm{d} x=\mathrm{d} x_{0}+\mathrm{d} s\left(x_{0}\right)$. The $\mathrm{d} s$ integral is $\mathrm{O}(\varepsilon)$ smaller than the $\mathrm{d} x_{0}$ integral, hence it is negligible. Therefore we estimate now

$$
\begin{aligned}
\sigma_{m} & =\varepsilon \mathrm{i} \frac{\beta_{m}}{k_{m} L} \int_{0}^{L} \frac{\delta v_{+}(t, x)}{v_{1}-v_{0}} \mathrm{e}^{-\mathrm{i} k_{m} x+\mathrm{i} \omega_{m} t} \mathrm{~d} x \\
& \simeq \varepsilon \mathrm{i} \frac{\beta_{m}}{k_{m} L} \int_{0}^{L} \frac{w\left(t, x_{0}\right)}{v_{1}-v_{0}} \mathrm{e}^{-\mathrm{i} k_{m} x_{0}-\mathrm{i} \Omega_{m} t} \mathrm{~d} x_{0}
\end{aligned}
$$

The space dependence of $w$, due to $\alpha_{p}=\beta_{p} \zeta_{p} \mathrm{e}^{\mathrm{i} k_{p} x_{0}}$, implies that $w_{1}$ contributes to $\sigma_{m}$ only through the $\ell=m$ spatial component. This contribution $\sigma_{m, 1}=\mathrm{O}\left(\varepsilon^{2}\right)$ does not oscillate as its time dependence fullfils the resonance condition $\Omega_{\ell}=\Omega_{m}$ because of the specific dispersion relation, $\omega_{m}=\omega_{\mathrm{p}}, k_{m}=\left(m+\nu_{0}\right) 2 \pi L^{-1}$. However, it involves no other wave than $m$, and does not couple waves nonlinearly.

The $w_{2}$ contribution to $\sigma_{m}$ oscillates in time, because any waves $\ell, p$, meeting the spatial resonance condition $k_{m}=c_{\ell} k_{\ell}+c_{p} k_{p}$ (imposed by the space integral over $x_{0}$ ) for some $c_{\ell}, c_{p} \in\{-1,1\}$, will verify $c_{\ell} \Omega_{\ell}+c_{p} \Omega_{p}=k_{m} v_{1}-\left(c_{\ell}+c_{p}\right) \omega_{\mathrm{p}}=\Omega_{m}+\left(1-c_{\ell}-c_{p}\right) \omega_{\mathrm{p}}$. Hence they cannot meet the time-resonance condition $\Omega_{m}=c_{\ell} \Omega_{p}+c_{p} \Omega_{\ell}$.

Most terms in $w_{3}$ also generate time-oscillating terms in $\sigma_{m}$, but some waves $\ell, p, q$ do fullfil the non-oscillation condition $\Omega_{m}=\Omega_{p}+\Omega_{q}-\Omega_{\ell}$, and these triplets automatically meet the spatial resonance condition $k_{m}=k_{p}+k_{q}-k_{\ell}$. These four-wave coupling terms occur in a degenerate form, as any pair $p, q \in \mathbb{Z}$ defines a resonant partner $\ell=p+q-m$. The four-wave coupling terms in $\sigma$ add up to

$$
\sigma_{m}^{\mathrm{res}}=\frac{\varepsilon^{4} \beta_{m}}{16 k_{m}\left(v_{1}-v_{0}\right)} \sum_{p, q, \ell} \beta_{p} \beta_{q} \beta_{\ell} C_{p q} \zeta_{\ell}^{*} \zeta_{p} \zeta_{q}
$$

with

$$
\begin{aligned}
C_{p q}= & -\left(k_{\ell}^{2} \Omega_{p}^{-2} \Omega_{q}^{-2}+k_{q}^{2} \Omega_{\ell}^{-2} \Omega_{p}^{-2}+k_{p}^{2} \Omega_{q}^{-2} \Omega_{\ell}^{-2}\right)+2 k_{\ell} k_{p} \Omega_{q}^{-2}\left(\Omega_{p}+\Omega_{q}\right)^{-2} \\
& +2\left(1-\delta_{\ell p}\right) C_{p \ell}^{\prime} k_{q}
\end{aligned}
$$

where $\ell=p+q-m$ and

$$
\begin{aligned}
C_{p \ell}^{\prime} & =\left(\Omega_{\ell}-\Omega_{p}\right)^{-2}\left(k_{\ell} \Omega_{p}^{-2}-k_{p} \Omega_{\ell}^{-2}\right) \\
& =\left(\Omega_{\ell}-\Omega_{p}\right)^{-1} v_{1}^{-1} \Omega_{\ell}^{-2} \Omega_{p}^{-2}\left(\Omega_{\ell}^{2}+\Omega_{\ell} \Omega_{p}+\Omega_{p}^{2}+\Omega_{\mathrm{p}}\left(\Omega_{\ell}+\Omega_{p}\right)\right)
\end{aligned}
$$

In the four-wave coupling coefficient $C_{p q}$ all terms are order unity in the limit of a dense spectrum, except those contributing to $C_{p q}^{\prime}$. In the latter terms the worst divergence scales like $\left(\Omega_{p+1}-\Omega_{p}\right)^{-1}=L /\left(2 \pi v_{1}\right)$, for $\ell=p \pm 1$, coupling waves with nearest wavenumbers (and nearly equal phase velocities). The other terms are milder, and the resulting, harmonic-like truncated series with coefficients $\left(\Omega_{p+1}-\Omega_{p}\right)^{-1}$ will typically involve wave envelope factors $\zeta_{\ell}^{*} \zeta_{p} \zeta_{q}$ with incoherent phases, ensuring its convergence. 
Therefore, with all $k$ 's having comparable scales, the four-wave coupling term scales like a sum of $\varepsilon^{4} \beta^{4}|\zeta|^{3} /\left[v_{1}\left(v_{1}-v_{0}\right) \Omega_{\min }^{2} \delta \Omega\right]$ and $\varepsilon^{4} \beta^{4}|\zeta|^{3} k /\left[\left(v_{1}-v_{0}\right) \Omega_{\text {min }}^{4}\right]$. A similar argument applies to the slower boundary particles, with average velocity $v_{0}>0$.

\section{References}

[1] Adam J C, Laval G and Pesme D 1979 Reconsideration of quasilinear theory Phys. Rev. Lett. 43 1671-5

[2] Antoni M, Elskens Y and Escande D F 1998 Explicit reduction of $N$-body dynamics to selfconsistent particle-wave interaction Phys. Plasmas 5 841-52

[3] Bechouche P and Besse N 2010 Analysis of a semi-Lagrangian method for the spherically symmetric Vlasov-Einstein system ESAIM: M2AN 44 573-95

[4] Bénisti D and Escande D F 1997 Origin of diffusion in Hamiltonian dynamics Phys. Plasmas 4 1576-81

[5] Bénisti D and Escande D F 1998 Finite range of large perturbations in Hamiltonian dynamics J. Stat. Phys. 92 909-72

[6] Bénisti D and Escande D F 1998 Nonstandard diffusion properties of the standard map Phys. Rev. Lett. $804871-4$

[7] Besse N 2004 Convergence of a semi-Lagrangian scheme for the one-dimensional Vlasov-Poisson system SIAM J. Numer. Anal. 42 350-82

[8] Besse N and Mehrenberger M 2008 Convergence of classes of high-order semi-Lagrangian schemes for the Vlasov-Poisson system Math. Comp. 77 93-123

[9] Besse N 2008 Convergence of a high-order semi-Lagrangian scheme with propagation of gradients for the Vlasov-Poisson system SIAM J. Numer. Anal. 46 639-70

[10] Besse N and Sonnendrücker E 2003 Semi-Lagrangian schemes for the Vlasov equation on an unstructured mesh of phase space J. Comput. Phys. 191 341-76

[11] Boutros-Ghali T and Dupree T H 1981 Theory of two-point correlation function in Vlasov plasma Phys. Fluids 24 1839-58

[12] Cary J R, Doxas I, Escande D F and Verga A D 1992 Enhancement of the velocity diffusion in longitudinal plasma turbulence Phys. Fluids B 4 2062-69

[13] Cary J R, Escande D F and Verga A D 1990 Non quasilinear diffusion far from chaotic threshold Phys. Rev. Lett. 65 3132-5

[14] Cheng C Z and Knorr G 1976 The integration of the Vlasov equation in configuration space $J$. Comput. Phys. 22 330-51

[15] Doxas I and Cary J R 1997 Numerical observation of turbulence enhanced growth rates Phys. Plasmas 4 2509-18

[16] Drummond W E and Pines D 1962 Nonlinear stability of plasma oscillations Nucl. Fusion Suppl. 3 1049-57

[17] Dupree T H 1966 A perturbation theory for strong plasma oscillations Phys. Fluids 9 1773-82

[18] Dupree T H 1972 Theory of phase space density granulation in plasma Phys. Fluids 15 334-44

[19] Elskens Y 2007 Quasilinear limit for particle motion in a prescribed spectrum of random waves Physics AUC 17 (I) 109-21

[20] Elskens Y 2010 Nonquasilinear evolution of particle velocity in incoherent waves with random amplitudes Commun. Nonlinear Sci. Numer. Simul. 15 10-5

[21] Elskens Y and Escande D 2003 Microscopic dynamics of plasmas and chaos (Bristol: IoP Publishing)

[22] Elskens Y and Firpo M-C 1998 Kinetic theory and large- $N$ limit for wave-particle self-consistent interaction Phys. Scr. T 75 169-72

[23] Elskens Y and Pardoux E 2010 Diffusion limit for many particles in a periodic stochastic acceleration field Ann. Appl. Prob. (in press) 
[24] Escande D F 1989 Description of Landau damping and weak Langmuir turbulence through microscopic dynamics Nonlinear world vol 2 ed V G Bar'yakhtar, V M Chernousenko, N S Erokhin, A G Sitenko and V E Zakharov (Singapore: World Scientific) pp 817-36

[25] Escande D F 1991 Large scale structures in kinetic plasma turbulence Large scale structures in nonlinear physics (Lect. Notes Phys. vol 392) ed J D Fournier and P L Sulem (Berlin: Springer) pp 73-104

[26] Escande D F 2010 Wave-particle interaction in plasmas : A qualitative approach Long-range interacting systems ed T Dauxois, S Ruffo and L F Cugliandolo (Oxford: Oxford University press) pp 817-36

[27] Escande D and Elskens Y 2002 Quasilinear diffusion for the chaotic motion of a particle in a set of longitudinal waves Acta Phys. Pol. B 33 1073-84

[28] Escande D and Elskens Y 2002 Proof of quasilinear equations in the chaotic regime of the weak warm beam instability Phys. Lett. A 302 110-9

[29] Escande D and Elskens Y 2003 Proof of quasilinear equations in the strongly nonlinear regime of the weak warm beam instability Phys. Plasmas 10 1588-94

[30] Escande D and Elskens Y 2003 Microscopic dynamics of plasmas and chaos : the wave-particle interaction paradigm Plasma Phys. Control. Fusion 45 A115-24

[31] Escande D and Elskens Y 2008 Self-consistency vanishes in the plateau regime of the bump-on-tail instability preprint arXiv : 0807.1839 [nlin.CD]

[32] Feix M R and Bertrand P 2005 A universal model: The Vlasov equation Trans. Theo. Stat. Phys. 34 7-62

[33] Firpo M-C, Doveil F, Elskens Y, Bertrand P, Poleni M and Guyomarc'h D 2001 Long-time discrete particle effects versus kinetic theory in the self-consistent single-wave-particle interaction Phys. Rev. E 65026407

[34] Firpo M-C and Elskens Y 1998 Kinetic theory of $N$-body description of wave-particle selfconsistent interaction J. Stat. Phys. 93 193-209

[35] Galeev A A, Sagdeev R Z, Shapiro V D and Shevchenko V I 1980 Is renormalization necessary in the quasilinear theory of Langmuir oscillations? Zh. Eksp. Teor. Fiz. 79 (6) 2167-74 (English transl. Sov. Phys. JETP 52 1095-9)

[36] Hartmann D A, Driscoll C F, O'Neil T M and Shapiro V D 1995 Measurements of the warm beam instability Phys. Plasmas 2 654-77

[37] Landau L D 1946 On the vibrations of the electronic plasma Zh. Eksp. Teor. Fiz. 16 574-86 reprinted in Collected papers of Landau ed D ter Haar (Oxford: Pergamon, 1965) pp 445-60

[38] Laval G and Pesme D 1983 Breakdown of quasilinear theory for incoherent 1-D Langmuir waves Phys. Fluids 26 52-65

[39] Laval G and Pesme D 1983 Inconsistency of quasilinear theory Phys. Fluids 26 66-8

[40] Laval G and Pesme D 1984 Self-consistency effects in quasilinear theory : A model for turbulent trapping Phys. Rev. Lett. 53 270-3

[41] Laval G and Pesme D 1999 Controversies about quasi-linear theory Plasma Phys. Control. Fusion 41 A239-46

[42] Liang Y-M and Diamond P H 1993 Weak turbulence theory of Langmuir waves : a reconsideration of the validity of quasilinear theory Comments Plasma Phys. Controlled Fusion 15 139-49

[43] Liang Y-M and Diamond P H 1993 Revisiting the validity of quasilinear theory Phys. Fluids B 5 4333-40

[44] Malmberg J H and Wharton C B 1964 Collisionless damping of electrostatic plasma waves Phys. Rev. Lett. 13 184-6

[45] Mouhot C and Villani C 2009 On Landau damping Preprint arXiv:0904.2760

[46] Mouhot C and Villani C 2010 Landau damping J. Math. Phys. 51015204

[47] Mynick H E and Kaufman A N 1978 Soluble theory of nonlinear beam-plasma interaction Phys. Fluids 21 653-63

[48] O'Neil T M, Winfrey J H and Malmberg J H 1971 Nonlinear interaction of a small cold beam 
and a plasma Phys. Fluids 14 1204-12

[49] Onishchenko I N, Linetski A R, Matsiborko N G, Shapiro V D and Shevchenko V I 1970 Contribution to the nonlinear theory of excitation of a monochromatic plasma wave by an electron beam ZhETF Pis. Red. 12 407-11 (Eng. transl. JETP Lett. 12 281-5)

[50] Roberson C and Gentle K W 1971 Experimental test of the quasilinear theory of the gentle bump instability Phys. Fluids 14 2462-9

[51] Shapiro V D and Sagdeev R Z 1997 Nonlinear wave-particle interaction and conditions for applicability of quasilinear theory Phys. Rep. 283 49-71

[52] Spohn H 1991 Large scale dynamics of interacting particles (Berlin: Springer)

[53] Tennyson J L, Meiss J D and Morrison P J 1994 Self-consistent chaos in the beam-plasma instability Physica D 71 1-17

[54] Tsunoda S I, Doveil F and Malmberg J H 1991 Experimental test of quasilinear theory Phys. Fluids B $32747-57$

[55] Vedenov A A, Velikhov E P and Sagdeev R Z 1962 Quasilinear theory of plasma oscillations Nucl. Fusion Suppl. 2 465-75 